\title{
Structural Change tests based on Implied Probabilities for GEL Criteria
}

\author{
Alain Guay ${ }^{a}$, Jean-François Lamarche $e^{b, \dagger}$ \\ ${ }^{a}$ Département de sciences économiques, Université du Québec à Montréal, Québec, Canada. CIRPÉE and \\ CIREQ. E-mail: guay.alain@uqam.ca \\ ${ }^{b}$ Department of Economics, Brock University, St. Catharines, Ontario, Canada. E-mail: jf@@rocku.ca
}

May 20, 2009

\begin{abstract}
$\dagger$ A previous version, October 2008, of this paper was originally circulated under the title: "The Information Content of Implied Probabilities to Detect Structural Change". The first author gratefully acknowledges financial support of le Fonds québécois de la recherche sur la société et la culture (FQRSC). The first and second authors are thankful for the financial support of the Social Sciences and Humanities Research Council of Canada (SSHRC). We are grateful to Chuan Goh for his comments and also for the comments of participants at the CIREQ Conference on Generalized Method of Moments, Montréal, 2007, ESEM Budapest 2007 and CEA meeting 2007.
\end{abstract}


Running Head: Probabilities to Detect Structural Change

\begin{abstract}
This paper proposes Pearson-type statistics based on implied probabilities to detect structural change. The class of generalized empirical likelihood estimators (see Smith (1997)) assigns a set of probabilities to each observation such that moment conditions are satisfied. These probabilities are called implied probabilities. The proposed test statistics for structural change are based on the information content in these implied probabilities. We consider cases of structural change with unknown breakpoint which can occur in the parameters of interest or in the overidentifying restrictions used to estimate these parameters. We also propose a structural change test based on implied probabilities that is robust to weak identification or cases in which parameters are completely unidentified. The test statistics considered here have good size and competitive power properties. Moreover, they are computed in a single step which eliminates the need to compute the weighting matrix required for GMM estimation.
\end{abstract}

Keywords: Generalized empirical likelihood, generalized method of moments, parameter instability, structural change.

JEL codes: C12, C32

Corresponding Author:

Jean-François Lamarche

Department of Economics, Brock University

500 Glenridge Avenue, St. Catharines

Ontario, Canada, L2S 3A1

Phone: 905-688-5550 ext. 3328

E-mail: jfl@brocku.ca 


\section{Introduction}

This paper proposes structural change tests based on implied probabilities resulting from estimation methods based on unconditional moment restrictions. The generalized method of moments

(GMM) is the standard method to estimate parameters through moment restrictions. Monte Carlo results reveal, however, that GMM estimators may be seriously biased in small samples. ${ }^{1}$ Recently, a number of alternative estimators to GMM have been proposed. Hansen, Heaton and Yaron (1996) suggested the continuous updating estimator (CUE) which shares the same objective function as GMM but with a weighting matrix that depends on the parameters of interest. The empirical likelihood (EL) (see Qin and Lawless (1994)) and the exponential tilting (ET) estimators (see Kitamura and Stutzer (1997)) have also been proposed. Kitamura (2001) showed that tests for the validity of moment restrictions based on EL have optimal properties in terms of large deviations. In particular, EL-based tests are shown to be more powerful than other standard tests. These alternative estimators are special cases of the generalized empirical likelihood (GEL) class considered by Smith (1997) and may be shown to be based on the Cressie and Read (1984) family of power divergence criteria. Newey and Smith (2004) showed (in an i.i.d. setting) that, although estimators based on GMM, EL, ET or CU have the same first-order asymptotic distribution, they have different higher-order asymptotic properties. Amongst their findings, it is shown that the expression for the second-order asymptotic bias of GEL has fewer components than the one of GMM (with EL having the fewest). Anatolyev (2005) extended the Newey and Smith setting to allow for weakly dependent data correlation and show that the asymptotic bias of smoothed GEL estimators is less than GMM estimators, especially with many moment conditions.

GEL estimators assign a probability to each observation such that the moment conditions are satisfied (see Smith (2004)). This resulting empirical measure is called implied probabilities. An interpretation of the implied probabilities is the following: less weight is assigned to an observation for which the moment restrictions are not satisfied at the estimated values of the parameters and more weight to an observation for which the moment restrictions are satisfied. As suggested by Back and Brown (1993), implied probabilities may then provide a useful diagnostic device for model specification. In particular, implied probabilities may contain interesting information to detect instability in the sample. Consequently, we propose to use these weights in detecting an unknown structural change in the model. Antoine, Bonnal and Renault (2007) use the weights given by the implied probabilities to propose a three-step estimator with higher-order asymptotic properties equivalent to those of EL. Schennach (2007) also discusses the use of these weights in the context of model misspecification. Ramalho and Smith (2005) considered Pearson-type test statistics (statistics based on the difference between restricted and unrestricted estimators of the weights) for the validity of moment restrictions 
and parametric restrictions using implied probabilities.

The proposed test statistics to detect structural change are based on different measures of the discrepancy between these implied probabilities and the unconstrained empirical probabilities $\frac{1}{T}(T$ is the sample size). The test statistics fall in three categories: 1) general structural change tests to detect instability in the identifying restrictions and in the overidentifying restrictions; 2) structural change tests specifically designed to detect instability in the identifying restrictions (see for example Andrews (1993)) and 3) structural change tests to detect instability in the overidentifying restrictions (see for example Hall and Sen (1999)). As an additional contribution of this paper, we propose a boundedly pivotal structural change test based on implied probabilities robust to cases of weak identification or cases in which parameters are completely unidentified. In particular, our derivation allows us to examine the power of the test under different assumptions about identification, and so for general alternatives about parameters instability. The asymptotic distributions of the test statistics are derived under the null hypothesis and under the alternative of an unknown breakpoint. In a simulation study, we find that targeted tests based on implied probabilities performed very well if the structural change corresponds to the target. That is, if instability is present in the identifying restrictions or in the overidentifying restrictions, then the targeted tests have good size and competitive power properties compared to more standard structural change tests for GEL criteria (see Guay and Lamarche (2009)). Overall, the test statistics based on implied probabilities considered in this paper have a nice intuitive appeal, are based on an estimation method that has been shown to have nice higher-order asymptotic properties and are computed in a single step which eliminates the need to compute the weighting matrix required to perform alternative structural change tests in GEL or GMM settings. This weighting matrix is often linked to the poor finite sample properties of GMM-based estimators and test statistics.

The paper is organized as follows. A discussion on GMM and GEL estimators are presented in section 2. Section 3 presents formally the full-sample and partial-sample GMM and GEL estimators. Section 4 presents the test statistics proposed based on the implied probabilities. The simulation results are in Section 5 while the proofs are in the Appendix.

\section{Discussion of GMM and GEL estimators}

In this section we present the estimators used in this paper. We start with an entropy-based formulation of the problem which puts emphasis on the informational content of the estimated weights. We then move to the more recent GEL formulation (see Newey and Smith (2004) and Smith (2004)).

We consider a triangular array of random variables $\left\{x_{T t}: 1 \leq t \leq T, T \geq 1\right\}$. Triangular arrays are 
considered because they are required to examine the results under local alternatives. For notational simplicity $x_{t}$ refers to $x_{T t}$ hereafter. Suppose we have a $q \times 1$ vector function of the data, $g\left(x_{t}, \theta\right)$, which depends on some unknown $p$-vector of parameters $\theta \in \Theta$ with $\Theta \subset R^{p}$ and that in the population their expected value is 0 , namely

$$
E\left[g\left(x_{t}, \theta_{0}\right)\right]=0
$$

We consider the overidentifying case with $q>p$.

The standard GMM estimators (Hansen (1982)) are obtained as the solution of

$$
\tilde{\theta}_{T}=\arg \min _{\theta \in \Theta} g_{T}(\theta)^{\prime} W_{T} g_{T}(\theta)
$$

where $W_{T}$ is a random, positive definite symmetric $q \times q$ matrix and $g_{T}(\theta)=\frac{1}{T} \sum_{t=1}^{T} g\left(x_{t}, \theta\right)$. The optimal weighting matrix is defined to be the inverse of the covariance matrix of the moment conditions, $W_{T}=\Omega_{T}^{-1}$, where $\Omega_{T}$ is a consistent estimator of

$$
\Omega=\lim _{T \rightarrow \infty} \operatorname{Var}\left(\frac{1}{\sqrt{T}} \sum_{t=1}^{T} g\left(x_{t}, \theta\right)\right) .
$$

The optimal weighting matrix can be estimated consistently using methods developed by Gallant (1987), Andrews and Monahan (1992) and Newey and West (1994), amongst several others. Importantly, this weighting matrix must be estimated once for the case of the two-step GMM estimator and more for the CUE.

We note that, in the GMM criterion function, the moment conditions receive equal weight $(1 / T)$ for each observations. Back and Brown (1993) derive a set of implicit weights using the GMM estimators given by

$$
\pi_{t}(\theta)=\frac{1}{T}-\frac{1}{T-p}\left[J_{t T}(\theta)-J_{T}(\theta)\right]^{\prime} \hat{\Omega}_{T}(\theta)^{-1} g_{T}(\theta)
$$

with $J_{T}(\theta)=\frac{1}{T} \sum_{t=1}^{T} J_{t T}(\theta)$ and

$$
J_{t T}(\theta)=\sum_{j=1}^{T} \kappa(|t-j|) g\left(x_{t-j}, \theta\right)
$$

where $\kappa(|t-j|)$ is a real valued weighting function. The following estimator

$$
\hat{\Omega}_{T}(\theta)=\frac{1}{T-p} \sum_{t=1}^{T} J_{t T}(\theta) g\left(x_{t}, \theta\right)^{\prime}
$$

is a consistent and positive definite estimator of the variance-covariance matrix and it has the usual form of a heteroskedasticity and autocorrelation consistent (HAC) weight matrix for standard kernels $\kappa(|t-s|)$ considered in the vast literature on consistent estimation of variance-covariance matrix (e.g. Andrews (1991), Newey and West (1987) and Newey and West (1994)). The implicit probabilities 
defined above are the empirical measure that ensures that the moment conditions are satisfied in the sample.

Now letting the $T$-vector of explicit weights (explicit because the weights are used directly in estimation) be $\left\{\pi_{t}: 1 \leq t \leq T, T \geq 1\right\}$ we can recast the population moment conditions as

$$
E_{\pi}\left[g\left(x_{t}, \theta_{0}\right)\right]=0
$$

The vector $\pi$ is determined by finding the most probable data distribution of the outcomes given the data. We can think of $\pi$ as containing information on the content of the moment conditions. Therefore, $g(x, \theta)$ is viewed as a message. That is, when $\pi$ is small, the message is informative and vice-versa. This relation is summarized by the function $f(\pi)=-\ln \pi$. The average information is then

$$
S(\pi) \equiv E_{\pi} f(\pi)=-\sum_{t=1}^{T} \pi_{t} \ln \pi_{t}
$$

In this case, $S(\pi)$ can be interpreted as the entropy measure of Shannon (1948) and it captures the degree of uncertainty in the distribution $\pi$ with respect to whether or not the distribution is concentrated or dispersed. The vector $\pi$ is obtained by maximizing entropy

$$
\max S(\pi)=-\sum_{t=1}^{T} \pi_{t} \ln \pi_{t}
$$

subject to $\sum_{t=1}^{T} \pi_{t}=1$ and $\sum_{t=1}^{T} \pi_{t} g\left(x_{t}, \theta\right)=0$.

With no constraint, we get $\pi_{t}=1 / T \quad \forall t$, the maximally uninformative uniform distribution, while with constraints, we want to choose $\pi_{t}$ to be as maximally uninformative as the moment conditions will allow. We do not want to assert more about the distribution than is known via the moment conditions. In this sense, the probabilities make use of all the information that is available, and nothing more. In particular, we focus on detecting a structural change in the moment conditions. With no structural change, the weights will fluctuate around $1 / T$, otherwise the entropy formulation will attempt to reduce the weight on the observation characterized by the change, and at the same time put more weights on the remaining observations so as to make $S(\pi)$ as large as possible.

We can transpose this formulation using the Kullback and Leibler (1951) information criterion which measures the discrepancy between two distributions $p$ and $\pi$. If the subject distribution is $\pi$ and the reference distribution is $p_{t}=1 / T, \forall t$ we have

$$
K L I C(\pi, p=1 / T)=\sum_{t=1}^{T} \pi_{t}\left[\ln \left(\pi_{t}\right)-\ln (1 / T)\right]=\sum_{t=1}^{T} \pi_{t} \ln \left(\pi_{t}\right)+\ln (T) .
$$

So that maximizing entropy is equivalent to minimizing the $K L I C(\pi, p=1 / T)$. Estimates of $\pi$, given $\theta$, are obtained by maximizing $S(\pi)$ (or by minimizing $K L I C(\pi, p=1 / T)$ ) subject to the 
weighted zero functions and the probability constraint. The solution to the Lagrangian yields

$$
\pi_{t}^{E T}(\theta)=\frac{\exp \left(\gamma^{\prime} g\left(x_{t}, \theta\right)\right)}{\sum_{t=1}^{T} \exp \left(\gamma^{\prime} g\left(x_{t}, \theta\right)\right)}
$$

where the $q$-vector $\gamma$ contains the Lagrange multipliers and as such measures the degree of departure from zero of the moment conditions and ET stands for exponential tilting. Estimates of $\theta$ are obtained by substituting $\pi$ in $S(\pi)$, maximizing it with respect to $\gamma$ and then with respect to $\theta$ (see for example Kitamura and Stutzer (1997)).

If we interchange the subject/reference distributions we get

$$
K L I C(p=1 / T, \pi)=\sum_{t=1}^{T}(1 / T)\left[\ln (1 / T)-\ln \left(\pi_{t}\right)\right]
$$

and the solution to the optimization problem yields a different set of weights given by

$$
\pi_{t}^{E L}(\theta)=\frac{1}{T\left[1+\gamma^{\prime} g\left(x_{t}, \theta\right)\right]}
$$

where $E L$ stands for empirical likelihood. When we evaluate the weights at some estimators we obtain $\pi_{t}^{E T}\left(\tilde{\theta}_{T}\right)$ and $\pi_{t}^{E L}\left(\tilde{\theta}_{T}\right)$. Recently, Schennach (2007) combined ET and EL into the ETEL estimator that combines the advantages of each approach.

We mentioned in the introduction that less weight is assigned to an observation for which the moment conditions are not satisfied. In this section we have seen that the vector $\pi$ contains all the relevant information with respect to the moment conditions. We now provide a graphical illustration of the use of the weights in the detection of a structural change. We consider a small simulation study that contains three examples that have been studied in the structural change and entropy literature. The first example, which encompasses three cases, is similar to the one used by Imbens, Spady and Johnson (1998) and consists of estimating a single parameter, $\theta$, with 2 moment conditions:

$$
E\left[x_{t}-\theta\right]=0 \text { and } E\left[\left(x_{t}-\theta\right)^{2}-4\right]=0
$$

with a sample of 100 observations and $x_{t}=\theta_{t}+\epsilon_{t}$ where $\epsilon_{t} \sim N(0,4)$. We consider a pulse, a break and a regime shift cases. In the pulse case we have $\theta_{t}=5$ for $t=50$ and $\theta=10$ otherwise. For the break case we have $\theta_{t}=10$ for $t \leq 20$ and $t>80$ while $\theta_{t}=15$ for $21 \leq t \leq 80$. Finally, the regime shift case has $\theta_{t}=10$ for $t \leq 20$ and $\theta_{t}=15$ otherwise. In these cases, structural change occurs via the parameters and can be tested using procedures proposed by Andrews (1993) and by Andrews and Ploberger (1994).

In contrast, the next two examples consist of structural change through the moment conditions. Following Hall and Horowitz (1996) and Gregory, Lamarche and Smith (2002) we study a simulated environment with CRRA preferences and making a distributional assumption on consumption growth, $x_{t}$, with i.i.d data and $T=100$. In particular, we assume that consumption growth follows a $N\left(0, \sigma^{2}=\right.$ 
0.16). There is a single parameter to be estimated, $\gamma$, the coefficient of CRRA and two moment conditions are used:

$$
\begin{aligned}
E_{t} \exp \left[-\gamma \ln x_{t+1}-9 \sigma^{2} / 2+(3-\gamma) z_{t}\right] & =1 \\
E_{t} z_{t} \exp \left[-\gamma \ln x_{t+1}-9 \sigma^{2} / 2+(3-\gamma) z_{t}-1\right] & =0
\end{aligned}
$$

with $z_{t} \sim N\left(0, \sigma^{2}\right)$. The moment conditions are satisfied when $\gamma=3$. The structural break occurs in period 51 and is summarized by a shift in $\gamma$ from 3 to 4 .

Lastly, as in Ghysels, Guay and Hall (1997), we have the estimation of an autoregressive parameter using two moment conditions when the data generating process is an $A R(1)\left(x_{t}=\rho x_{t-1}+\epsilon_{t}\right)$, for $t \leq 50$, and an $\operatorname{ARMA}(1,2)$ otherwise $\left(x_{t}=\rho x_{t-1}+\epsilon_{t}+0.5 \epsilon_{t-2}\right)$. There are 100 observations and $\epsilon_{t} \sim N(0,1)$. The two instruments used are the first and second lags of $x_{t}$. The two moment conditions are then:

$$
\begin{aligned}
& E\left[x_{t-1}\left(x_{t}-\rho x_{t-1}\right)\right]=0 \\
& E\left[x_{t-2}\left(x_{t}-\rho x_{t-1}\right)\right]=0 .
\end{aligned}
$$

The instability occurs because the second moment condition is violated for $t>50$.

Figure 1 shows the average of the vector of implied probabilities $\pi$ over 10,000 replications. The key feature of these panels is that when there is no break, the weights fluctuate around $1 / T=1 / 100$ (upper right panel). With a structural break in the parameter or in the moment conditions, however, more weight is given to observations (and moment conditions) for which there is no break while less weight is assigned to an observation which violates the moment conditions. This simple simulation study clearly show that implied probabilities contain interesting information to detect structural change. In this paper, we examine the information contained in the estimated implied probabilities to detect structural change and propose test statistics based on some function of these implied probabilities.

Now following the recent econometric literature on GEL (see Newey and Smith (2004), Smith (2004), Ramalho and Smith (2005), Caner (2008a), Caner (2008b) and Guggenberger and Smith $(2008))$, we let $\rho(\phi)$ be a function of a scalar $\phi$ that is concave on its domain, an open interval $\Phi$ that contains 0 . Also, let $\tilde{\Gamma}_{T}(\theta)=\left\{\gamma: \gamma^{\prime} g\left(x_{t}, \theta\right) \in \Phi, t=1, \ldots, T\right\}$. Then, the GEL estimator is a solution to the problem

$$
\tilde{\theta}_{T}=\arg \min _{\theta \in \Theta} \sup _{\gamma \in \tilde{\Gamma}_{T}(\theta)} \sum_{t=1}^{T} \frac{\left[\rho\left(\gamma^{\prime} g\left(x_{t}, \theta\right)\right)-\rho_{0}\right]}{T}
$$

where $\rho_{j}()=\partial^{j} \rho() / \partial \phi^{j}$ and $\rho_{j}=\rho_{j}(0)$ for $j=0,1,2, \ldots$ Under this formulation, a number of estimators can be obtained. First, the ET estimator of $\theta$ is found by setting $\rho(\phi)=-\exp (\phi)$. Second, the EL estimator of $\theta$ by setting $\rho(\phi)=\ln (1-\phi)$. Third, the continuous updating estimator of Hansen 
et al. (1996) can also be obtained from the GEL formulation by using a quadratic function for $\rho(\phi)$, $-(1+\phi)^{2} / 2$.

As in the GMM context an adjustment for the dynamic structure of $g\left(x_{t}, \theta\right)$ can also be made in the GEL context (see Kitamura and Stutzer (1997), Smith (2000), Smith (2004) and Guggenberger and Smith (2008)). The adjustment consists of smoothing the original moment conditions $g\left(x_{t}, \theta\right)$. Defining the smoothed moment conditions as

$$
g_{t T}(\theta)=\frac{1}{R_{T}} \sum_{r=t-T}^{t-1} k\left(\frac{r}{R_{T}}\right) g\left(x_{t-r}, \theta\right)
$$

for $t=1, \ldots, T$ and $R_{T}$ is a bandwidth parameter, $k(\cdot)$ a kernel function and we define where $k_{j}=\int_{-\infty}^{\infty} k(a)^{j} d a$. In the time series context, the criteria is then given by:

$$
\sum_{t=1}^{T} \frac{\left[\rho\left(k \gamma^{\prime} g_{t T}(\theta)\right)-\rho_{0}\right]}{T}
$$

where $k=\frac{k_{1}}{k_{2}}($ see Smith $(2004))$.

\section{$3 \quad$ Full and partial-samples GMM and GEL estimators}

To establish the asymptotic distribution theory of tests for structural change in the parameters based on implied probabilities we need to elaborate on the specification of the parameter vector in our generic setup. We will consider parametric models indexed by parameters $(\beta, \delta)$. With no structural change we define a vector of parameters $(\beta, \delta) \subset B \times \Delta \in R^{p}$ with $p=r+\nu$. Following Andrews (1993), we make a distinction between pure structural change when no subvector $\delta$ appears and the entire parameter vector is subject to structural change under the alternative and partial structural change which corresponds to cases where only a subvector $\beta$ is subject to structural change under the alternative. The generic null can be written as follows:

$$
H_{0}: \beta_{t}=\beta_{0} \quad \forall t=1, \ldots, T
$$

The tests considered assume as alternative that at some point in the sample there is a single structural break. For example,

$$
\beta_{t T}= \begin{cases}\beta_{1}(s) & t=1, \ldots,[T s] \\ \beta_{2}(s) & t=[T s]+1, \ldots, T\end{cases}
$$

where $s$ determines the fraction of the sample before and after the assumed breakpoint and [.] denotes the greatest integer function. The separation $[T s]$ represents a possible breakpoint which is governed by an unknown parameter $s$. Hence, we will consider a setup with a parameter vector which encompasses any kind of partial or pure structural change involving a single breakpoint. In particular, we 
consider a $2 r+\nu$ dimensional parameter vector $\theta=\left(\beta_{1}^{\prime}, \beta_{2}^{\prime}, \delta^{\prime}\right)^{\prime}$ where $\beta_{1}$ and $\beta_{2} \in B \subset R^{r}$ and $\theta \in \Theta=B \times B \times \Delta \subset R^{2 r+\nu}$. The parameters $\beta_{1}$ and $\beta_{2}$ apply to the samples before and after the presumed breakpoint and the null implies that:

$$
H_{0}: \beta_{1}=\beta_{2}=\beta_{0}
$$

We will formulate all our models in terms of $\theta$. Special cases could be considered whenever restrictions are imposed in the general parametric formulation. One such restriction would be that $\theta_{0}=\left(\beta_{0}^{\prime}, \beta_{0}^{\prime}\right)^{\prime}$, which would correspond to the null of a pure structural change hypothesis. Once moment conditions are defined, we will translate them into a subspace identifying the parameters and a subspace for the overidentifying restrictions and relate these two translations to structural change tests, following the analysis of Sowell (1996a) and Hall and Sen (1999).

\subsection{Definitions}

We need to impose restrictions on the admissible class of functions and processes involved in estimation to guarantee well-behaved asymptotic properties of GMM and GEL estimators using the entire data sample or subsamples of observations. A set of regularity conditions is required to obtain weak convergence of partial-sample GMM and GEL estimators to a function of Brownian motions. To streamline the presentation we provide a detailed description of them in Appendix 7.1. We now formally define the standard GMM estimator using the full sample.

Definition 3.1. The full-sample General Method of Moments estimator $\left\{\left(\tilde{\beta}_{T}, \tilde{\delta}_{T}\right)\right\}$ is a sequence of random vectors such that:

$$
\left(\tilde{\beta}_{T}^{\prime}, \tilde{\delta}_{T}^{\prime}\right)^{\prime}=\arg \min _{(\beta, \delta) \in B \times \Delta} g_{T}(\beta, \delta)^{\prime} \hat{W}_{T} g_{T}(\beta, \delta)
$$

where $\hat{W}_{T}$ is a random positive definite symmetric $q \times q$ matrix.

The optimal weighting matrix $W$ is defined to be the inverse of $\Omega$ which is defined as:

$$
\Omega=\lim _{T \rightarrow \infty} \operatorname{Var}\left(\frac{1}{\sqrt{T}} \sum_{t=1}^{T} g\left(x_{t}, \beta_{0}, \delta_{0}\right)\right) .
$$

To characterize the asymptotic distribution we define the following matrices:

$$
\begin{gathered}
G_{\beta}=\lim _{T \rightarrow \infty} \frac{1}{T} \sum_{t=1}^{T} \operatorname{E\partial g}\left(x_{t}, \beta_{0}, \delta_{0}\right) / \partial \beta^{\prime} \in R^{q \times r}, \\
G_{\delta}=\lim _{T \rightarrow \infty} \frac{1}{T} \sum_{t=1}^{T} \operatorname{E} \partial g\left(x_{t}, \beta_{0}, \delta_{0}\right) / \partial \delta^{\prime} \in R^{q \times \nu}, \\
G=\left[\begin{array}{ll}
G_{\beta} & G_{\delta}
\end{array}\right] \in R^{q \times p},
\end{gathered}
$$


where $p=r+\nu$. Finally, let $\tilde{\theta}_{T}=\left(\tilde{\beta}_{T}^{\prime}, \tilde{\beta}_{T}^{\prime}, \tilde{\delta}_{T}^{\prime}\right)^{\prime}$ be a $2 r+\nu$-vector. Hereafter, the vector $\tilde{\theta}_{T}$ is called the full-sample estimator of $\theta$. This restricted estimator is consistent only under the null that $\beta_{1}=\beta_{2}$.

Several tests for structural change involving partial-sample GMM estimators were defined by Andrews (1993). We consider again the two subsamples, the first covers observations $t=1, \ldots,[T s]$ and the second covers $t=[T s]+1, \ldots, T$ where $s \in S \subset(0,1)$. The partial-sample GMM estimators for $s \in S$ based on the first and the second subsamples are formally defined as:

Definition 3.2. A partial-sample General Method of Moments estimator $\left\{\hat{\theta}_{T}(s)\right\}$ is a sequence of random vectors such that:

$$
\hat{\theta}_{T}(s)=\arg \min _{\theta \in \Theta} g_{T}(\theta, s)^{\prime} \hat{W}_{T}(s) g_{T}(\theta, s)
$$

for all $s \in S$. Define $g_{t}(\theta, s)=\left(g\left(x_{t}, \beta_{1}, \delta\right)^{\prime}, 0^{\prime}\right)^{\prime} \in R^{2 q \times 1}$ for $t=1, \ldots,[T s]$ and $g_{t}(\theta, s)=\left(0^{\prime}, g\left(x_{t}, \beta_{2}, \delta\right)^{\prime}\right)^{\prime} \in$ $R^{2 q \times 1}$ for $t=[T s]+1, \ldots, T$ such that

$$
g_{T}(\theta, s)=\frac{1}{T} \sum_{t=1}^{T} g_{t}(\theta, s)=\frac{1}{T} \sum_{t=1}^{[T s]}\left[\begin{array}{c}
g\left(x_{t}, \beta_{1}, \delta\right) \\
0
\end{array}\right]+\frac{1}{T} \sum_{t=[T s]+1}^{T}\left[\begin{array}{c}
0 \\
g\left(x_{t}, \beta_{2}, \delta\right)
\end{array}\right]
$$

and $\hat{W}_{T}(s)$ is a random positive definite symmetric $2 q \times 2 q$ matrix.

According to the definition above $\hat{\theta}_{T}(s)=\left(\hat{\beta}_{1 T}(s)^{\prime}, \hat{\beta}_{2 T}(s)^{\prime}, \hat{\delta}_{T}(s)^{\prime}\right)^{\prime}$ is a $2 r+\nu$-vector with an estimator $\hat{\beta}_{1 T}(s)$ that uses the first subsample $t=1, \ldots,[T s]$, an estimator $\hat{\beta}_{2 T}(s)$ that uses the second subsample $t=[T s]+1, \ldots, T$ and an estimator $\hat{\delta}_{T}(s)$ that uses all the sample.

The partial-sample optimal weighting matrix is defined as the inverse of $\Omega(s)$ where

$$
\Omega(s)=\lim _{T \rightarrow \infty} \operatorname{Var}\left(\frac{1}{\sqrt{T}}\left[\begin{array}{c}
\sum_{t=1}^{[T s]} g\left(x_{t}, \beta_{0}, \delta_{0}\right) \\
\sum_{t=[T s]+1}^{T} g\left(x_{t}, \beta_{0}, \delta_{0}\right)
\end{array}\right]\right)
$$

which under the null (3) is asymptotically equal to

$$
\Omega(s)=\left[\begin{array}{cc}
s \Omega & 0 \\
0 & (1-s) \Omega
\end{array}\right]
$$

We also define

$$
G(s)=\left[\begin{array}{ccc}
s G_{\beta} & 0 & s G_{\delta} \\
0 & (1-s) G_{\beta} & (1-s) G_{\delta}
\end{array}\right] \in R^{2 q \times(2 r+\nu)} .
$$

In the GEL setting, the parameter vector is augmented by a vector of auxiliary parameters $\gamma$ where each element of this vector is associated to an element of the smoothed moment conditions $g_{t T}(\theta)$. Under the null of no structural change relative to the specification of the model, the generic null hypothesis for this vector of auxiliary parameters can be written as follows:

$$
H_{0}: \gamma_{t}=\gamma_{0}=0 \quad \forall t=1, \ldots, T
$$


As for the parameter vector $\beta$, the tests that we will consider assume as alternative that at some point in the sample there is a single structural break, namely:

$$
\gamma_{t}= \begin{cases}\gamma_{1} & t=1, \ldots,[T s] \\ \gamma_{2} & t=[T s]+1, \ldots, T\end{cases}
$$

Thus, under the null of no structural change $H_{0}: \gamma_{1}=\gamma_{2}=\gamma_{0}=0$. Guay and Lamarche (2009) show that a structural change in $\gamma$ is associated with instability in the overidentifying restrictions.

We now formally define the GEL estimator using the full sample.

Definition 3.3. Let $\rho(\phi)$ be a function of a scalar $\phi$ that is concave on its domain, an open interval $\Phi$ that contains 0 . Also, let $\tilde{\Gamma}_{T}(\beta, \delta)=\left\{\gamma: k \gamma^{\prime} g_{t T}(\beta, \delta) \in \Phi, t=1, \ldots, T\right\}$ with $k=\frac{k_{1}}{k_{2}}$. Then, the full-sample GEL estimator $\left\{\tilde{\theta}_{T}\right\}$ is a sequence of random vectors such that:

$$
\left(\tilde{\beta}_{T}^{\prime}, \tilde{\delta}_{T}^{\prime}\right)^{\prime}=\arg \min _{(\beta, \delta) \in B \times \Delta} \sup _{\gamma \in \widetilde{\Gamma}_{T}(\beta, \delta)} \sum_{t=1}^{T} \frac{\left[\rho\left(k \gamma^{\prime} g_{t T}(\beta, \delta)\right)-\rho_{0}\right]}{T}
$$

where $\rho_{j}()=\partial^{j} \rho() / \partial \phi^{j}$ and $\rho_{j}=\rho_{j}(0)$ for $j=0,1,2, \ldots$.

The criteria is normalized so that $\rho_{1}=\rho_{2}=-1$ (see Smith (2004)). This GEL estimator encompasses the ET, EL and CU estimators, as mentioned earlier.

The GEL estimator is obtained as the solution to a saddle point problem. Firstly, the criterion is maximized for given vector $(\beta, \delta)$. Thus,

$$
\tilde{\gamma}_{T}(\beta, \delta)=\arg \sup _{\gamma \in \tilde{\Gamma}(\beta, \delta)} \sum_{t=1}^{T} \frac{\left[\rho\left(k \gamma^{\prime} g_{t T}(\beta, \delta)\right)-\rho_{0}\right]}{T} .
$$

Secondly, the GEL estimator $\left(\tilde{\beta}_{T}^{\prime}, \tilde{\delta}_{T}^{\prime}\right)^{\prime}$ is given by the following minimization problem:

$$
\left(\tilde{\beta}_{T}^{\prime}, \tilde{\delta}_{T}^{\prime}\right)^{\prime}=\arg \min _{(\beta, \delta) \in B \times \Delta} \sum_{t=1}^{T} \frac{\left[\rho\left(k \tilde{\gamma}_{T}(\beta, \delta)^{\prime} g_{t T}(\beta, \delta)\right)-\rho_{0}\right]}{T} .
$$

From now on, following Kitamura and Stutzer (1997) and Guggenberger and Smith (2008) we focus on the truncated kernel defined by

$$
k(x)=1 \text { if }|x| \leq 1 \text { and } k(x)=0 \text { otherwise }
$$

to obtain the following smoothed moment conditions

$$
g_{t T}(\beta, \delta)=\frac{1}{2 K_{T}+1} \sum_{j=-K_{T}}^{K_{T}} g\left(x_{t-j}, \beta, \delta\right) .
$$

To handle the endpoints, we use the approach of Smith (2004) and Guggenberger and Smith (2008) which sets

$$
g_{t T}(\beta, \delta)=\frac{1}{2 K_{T}+1} \sum_{j=\max \left\{t-T,-K_{T}\right\}}^{\min \left\{t-1, K_{T}\right\}} g\left(x_{t-j}, \beta, \delta\right) .
$$


We can easily show for this kernel that $k=\frac{k_{1}}{k_{2}}=1$. A consistent estimator of the long-run covariance matrix is then given by:

$$
\tilde{\Omega}_{T}=\frac{2 K_{T}+1}{T} \sum_{t=1}^{T} g_{t T}\left(\tilde{\beta}_{T}, \tilde{\delta}_{T}\right) g_{t T}\left(\tilde{\beta}_{T}, \tilde{\delta}_{T}\right)^{\prime} .
$$

The weighting matrix obtained using this type of kernel is similar to the one obtained with the Bartlett kernel estimator of the long-run covariance matrix of the moment conditions (see Smith (2004)). Define also the derivatives of the smoothed moment conditions as:

$$
G_{t T}(\beta, \delta)=\frac{1}{2 K_{T}+1} \sum_{j=-K_{T}}^{K_{T}} \frac{\partial g\left(x_{t-j}, \beta, \delta\right)}{\partial\left(\beta^{\prime}, \delta^{\prime}\right)} .
$$

Now consider a possible breakpoint $[T s]$. Define the vector of auxiliary parameters $\gamma(s)=\left(\gamma_{1}(s)^{\prime}, \gamma_{2}(s)^{\prime}\right)^{\prime}$ where $\gamma_{1}$ is the vector of the auxiliary parameters for the first part of the sample e.g. $t=1, \ldots,[T s]$ and $\gamma_{2}$ for the second part of the sample; $t=[T s]+1, \ldots, T$. The partial-sample GEL estimators for $s \in S$ based on the first and the second subsamples are formally defined as:

Definition 3.4. Let $\rho(\phi)$ be a function of a scalar $\phi$ that is concave on its domain, an open interval $\Phi$ that contains 0. Also, let $\hat{\Gamma}_{T}(\theta, s)=\left\{\gamma(s)=\left(\gamma_{1}(s)^{\prime}, \gamma_{2}(s)^{\prime}\right)^{\prime}: k \gamma(s)^{\prime} g_{t T}(\theta, s)\right\}$. A partial-sample General Empirical Likelihood (PS-GEL) estimator $\left\{\hat{\theta}_{T}(s)\right\}$ is a sequence of random vectors such that:

$$
\begin{aligned}
\hat{\theta}_{T}(s) & =\arg \min _{\theta \in \Theta} \sup _{\gamma(s) \in \hat{\Gamma}_{T}(\theta, s)} \sum_{t=1}^{T} \frac{\left[\rho\left(k \gamma(s)^{\prime} g_{t T}(\theta, s)\right)-\rho_{0}\right]}{T} \\
& =\arg \min _{\theta \in \Theta} \sup _{\gamma(s) \in \hat{\Gamma}_{T}(\theta, s)}\left(\sum_{t=1}^{[T s]} \frac{\left[\rho\left(k \gamma_{1}^{\prime} g_{t T}\left(\beta_{1}, \delta\right)\right)-\rho_{0}\right]}{T}+\sum_{t=[T s]+1}^{T} \frac{\left[\rho\left(k \gamma_{2}^{\prime} g_{t T}\left(\beta_{2}, \delta\right)\right)-\rho_{0}\right]}{T}\right)
\end{aligned}
$$

for all $s \in S$, where $g_{t T}(\theta, s)=\left(g_{t T}\left(\beta_{1}, \delta\right)^{\prime}, 0^{\prime}\right)^{\prime} \in R^{2 q \times 1}$ for $t=1, \ldots,[T s]$ and $g_{t T}(\theta, s)=$ $\left(0^{\prime}, g_{t T}\left(\beta_{2}, \delta\right)^{\prime}\right)^{\prime} \in R^{2 q \times 1}$ for $t=[T s]+1, \ldots, T$ with $\gamma(s)=\left(\gamma_{1}(s)^{\prime}, \gamma_{2}(s)^{\prime}\right)^{\prime} \in R^{2 q \times 1}$.

To be more precise, the first-order conditions corresponding to the Lagrange multiplier $\gamma$ are obtained from the maximization of the partial-sample GEL criterion for a given $\beta_{1}, \beta_{2}, \delta$. Thus,

$$
\begin{aligned}
\hat{\gamma}_{1 T}\left(\beta_{1}, \delta\right) & =\arg \sup _{\gamma_{1} \in \hat{\Gamma}_{1 T}\left(\beta_{1}, \delta\right)} \sum_{t=1}^{[T s]} \frac{\left[\rho\left(k \gamma_{1}\left(\beta_{1}, \delta\right)^{\prime} g_{t T}\left(\beta_{1}, \delta\right)\right)-\rho_{0}\right]}{T} \\
\hat{\gamma}_{2 T}\left(\beta_{2}, \delta\right) & =\arg \sup _{\gamma_{2} \in \hat{\Gamma}_{2 T}\left(\beta_{2}, \delta\right)} \sum_{t=[T s]+1}^{T} \frac{\left[\rho\left(k \gamma_{2}\left(\beta_{2}, \delta\right)^{\prime} g_{t T}\left(\beta_{2}, \delta\right)\right)-\rho_{0}\right]}{T} .
\end{aligned}
$$

with $\hat{\Gamma}_{1 T}\left(\beta_{1}, \delta\right)=\left\{\gamma_{1}: k \gamma_{1}^{\prime} g_{t T}\left(\beta_{1}, \delta\right) \in \Phi, t=1, \ldots,[T s]\right\}$ and $\hat{\Gamma}_{2 T}\left(\beta_{2}, \delta\right)=\left\{\gamma_{2}: k \gamma_{2}^{\prime} g_{t T}\left(\beta_{2}, \delta\right) \in\right.$ $\Phi, t=[T s]+1, \ldots, T\}$.

We also define the estimator

$$
\widehat{\Omega}_{T}(s)=\left[\begin{array}{cc}
s \widehat{\Omega}_{1 T}(s) & 0 \\
0 & (1-s) \widehat{\Omega}_{2 T}(s)
\end{array}\right] .
$$


with

$$
\widehat{\Omega}_{1 T}(s)=\frac{2 K_{T}+1}{[T s]} \sum_{t=1}^{[T s]} g_{t T}\left(\hat{\beta}_{1 T}(s), \hat{\delta}_{T}(s)\right) g_{t T}\left(\hat{\beta}_{1 T}(s), \hat{\delta}_{T}(s)\right)^{\prime}
$$

and

$$
\widehat{\Omega}_{2 T}(s)=\frac{2 K_{T}+1}{T-[T s]} \sum_{t=[T s]+1}^{T} g_{t T}\left(\hat{\beta}_{2 T}(s), \hat{\delta}_{T}(s)\right) g_{t T}\left(\hat{\beta}_{2 T}(s), \hat{\delta}_{T}(s)\right)^{\prime} .
$$

We now present the corresponding implied probabilities. The general formula of the implied probabilities for the full-sample GEL estimator is defined by the following ratio (see Smith, 2004):

$$
\pi_{t}^{G E L}\left(\tilde{\theta}_{T}\right)=\frac{\rho_{1}\left(\tilde{\gamma}_{T}^{\prime} g_{t T}\left(\tilde{\theta}_{T}\right)\right)}{\sum_{t=1}^{T} \rho_{1}\left(\tilde{\gamma}_{T}^{\prime} g_{t T}\left(\tilde{\theta}_{T}\right)\right)} .
$$

Implied probabilities for the full-sample ET, EL and CU estimators with the smoothed moment conditions are respectively given by:

and

$$
\begin{gathered}
\pi_{t}^{E T}\left(\tilde{\theta}_{T}\right)=\frac{\exp \left[\tilde{\gamma}_{T}^{\prime} g_{t T}\left(\tilde{\theta}_{T}\right)\right]}{\sum_{t=1}^{T} \exp \left[\tilde{\gamma}_{T}^{\prime} g_{t T}\left(\tilde{\theta}_{T}\right)\right]}, \\
\pi_{t}^{E L}\left(\tilde{\theta}_{T}\right)=\frac{1}{T\left[1+\tilde{\gamma}_{T}^{\prime} g_{t T}\left(\tilde{\theta}_{T}\right)\right]},
\end{gathered}
$$

$$
\pi_{t}^{C U E}\left(\tilde{\theta}_{T}\right)=\frac{1}{T}-\frac{1}{T} g_{t T}\left(\tilde{\theta}_{T}\right)\left[\frac{1}{T} \sum_{t-1}^{T} g_{t T}\left(\tilde{\theta}_{T}\right) g_{t T}\left(\tilde{\theta}_{T}\right)^{\prime}\right]^{-1} \frac{1}{T} \sum_{t=1}^{T} g_{t T}\left(\tilde{\theta}_{T}\right) .
$$

Note that $\frac{2 K+1}{T} \sum_{t-1}^{T} g_{t T}\left(\tilde{\theta}_{T}\right) g_{t T}\left(\tilde{\theta}_{T}\right)^{\prime}$ is a consistent estimator of $\Omega$.

The general formula for the unrestricted partial-sample implied probabilities for the GEL are defined for $s \in S$ as:

$$
\pi_{t}^{G E L}\left(\hat{\theta}_{T}(s), s\right)=\frac{\rho_{1}\left(\hat{\gamma}_{T}(s)^{\prime} g_{t T}\left(\hat{\theta}_{T}(s), s\right)\right)}{\sum_{t=1}^{T} \rho_{1}\left(\hat{\gamma}_{T}(s)^{\prime} g_{t T}\left(\hat{\theta}_{T}(s), s\right)\right)} .
$$

For example, in the case of $t$ between observations 1 and [Ts], we get for the unrestricted implied probabilities at $t$ :

$$
\pi_{t}^{G E L}\left(\hat{\beta}_{1 T}(s), \hat{\delta}_{T}(s), s\right)=\frac{\rho_{1}\left(\hat{\gamma}_{1 T}(s)^{\prime} g_{t T}\left(\hat{\beta}_{1 T}(s), \hat{\delta}_{T}(s)\right)\right)}{\sum_{t=1}^{T} \rho_{1}\left(\hat{\gamma}_{1 T}(s)^{\prime} g_{t T}\left(\hat{\beta}_{1 T}(s), \hat{\beta}_{2 T}(s), \hat{\delta}_{T}(s), s\right)\right)} .
$$

For the commonly used GEL partial-sample estimators, we get

$$
\begin{gathered}
\pi_{t}^{E T}\left(\hat{\theta}_{T}(s), s\right)=\frac{\exp \left[\hat{\gamma}_{T}(s)^{\prime} g_{t T}\left(\hat{\theta}_{T}(s), s\right)\right]}{\sum_{t=1}^{T} \exp \left[\hat{\gamma}_{T}(s)^{\prime} g_{t T}\left(\hat{\theta}_{T}(s), s\right)\right]}, \\
\pi_{t}^{E L}\left(\hat{\theta}_{T}(s), s\right)=\frac{1}{T\left[1+\hat{\gamma}_{T}(s)^{\prime} g_{t T}\left(\hat{\theta}_{T}(s), s\right)\right]},
\end{gathered}
$$




$$
\pi_{t}^{C U E}\left(\hat{\theta}_{T}(s), s\right)=\frac{1}{T}-\frac{1}{T} g_{t T}\left(\hat{\theta}_{T}(s), s\right)\left[\frac{1}{T} \sum_{t-1}^{T} g_{t T}\left(\hat{\theta}_{T}(s), s\right) g_{t T}\left(\hat{\theta}_{T}(s), s\right)^{\prime}\right]^{-1} \frac{1}{T} \sum_{t=1}^{T} g_{t T}\left(\hat{\theta}_{T}(s), s\right) .
$$

The purpose of the next subsection is to refine the null hypothesis of no structural change. Such a refinement will enable us to construct various tests for structural change in the spirit of Sowell (1996a) and Hall and Sen (1999).

\subsection{Refining the null hypothesis}

The moment conditions for the full sample under the null can be written as:

$$
E g\left(x_{t}, \theta_{0}\right)=0, \quad \forall t=1, \ldots, T
$$

Following Sowell (1996b), we can project the moment conditions on the subspace identifying the parameters and the subspace of overidentifying restrictions. In particular, considering the (standardized) moment conditions for the full-sample GMM estimator, such a decomposition corresponds to:

$$
\Omega^{-1 / 2} E g\left(x_{t}, \theta_{0}\right)=P_{G} \Omega^{-1 / 2} E g\left(x_{t}, \theta_{0}\right)+\left(I_{q}-P_{G}\right) \Omega^{-1 / 2} E g\left(x_{t}, \theta_{0}\right),
$$

where $P_{G}=\Omega^{-1 / 2} G\left[G^{\prime} \Omega^{-1} G\right]^{-1} G^{\prime} \Omega^{-1 / 2}$. The first term is the projection identifying the parameter vector and the second term is the projection for the overidentifying restrictions. The projection argument enables us to refine the null hypothesis (3). For instance, following Hall and Sen (1999) we can consider the null, for the case of a single possible breakpoint $s$, which separates the identifying restrictions across the two subsamples:

$$
H_{0}^{I}(s)=\left\{\begin{array}{llll}
P_{G} \Omega^{-1 / 2} E\left[g\left(x_{t}, \theta_{0}\right)\right]= & 0 & \forall t=1, \ldots,[T s] \\
P_{G} \Omega^{-1 / 2} E\left[g\left(x_{t}, \theta_{0}\right)\right]= & 0 & \forall t=[T s]+1, \ldots, T .
\end{array}\right.
$$

Moreover, the overidentifying restrictions are stable if they hold before and after the breakpoint. This is formally stated as $H_{0}^{O}(s)=H_{0}^{O 1}(s) \cap H_{0}^{O 2}(s)$ with:

$$
\begin{array}{lll}
H_{0}^{O 1}(s):\left(I_{q}-P_{G}\right) \Omega^{-1 / 2} E\left[g\left(x_{t}, \theta_{0}\right)\right]= & 0 & \forall t=1, \ldots,[T s] \\
H_{0}^{O 2}(s):\left(I_{q}-P_{G}\right) \Omega^{-1 / 2} E\left[g\left(x_{t}, \theta_{0}\right)\right]= & 0 & \forall t=[T s]+1, \ldots, T .
\end{array}
$$

The projection reveals that instability must be a result of a violation of at least one of the three hypotheses: $H_{0}^{I}(s), H_{0}^{O 1}(s)$ or $H_{0}^{O 2}(s)$. Various tests can be constructed with local power properties against any particular one of these three null hypotheses (and typically no power against the others). To elaborate further on this we consider a sequence of Pitman local alternatives based on the moment conditions:

Assumption 3.1. A sequence of local alternatives is specified as:

$$
E g\left(x_{T t}, \theta_{0}\right)=\frac{h\left(\eta, \tau, \frac{t}{T}\right)}{\sqrt{T}}
$$


where $h(\eta, \tau, s)$, for $r \in[0,1]$, is a $q$-dimensional function. The parameter $\tau$ locates structural changes as a fraction of the sample size and the vector $\eta$ defines the local alternatives. ${ }^{2}$ Note that the true structural change breakpoint $\tau$ is allowed to differ from the possible breakpoint $s$ chosen by the researcher. These local alternatives are chosen to show that the structural change tests presented in this paper have non trivial power against a large class of alternatives. Also, our asymptotic results can be compared with Sowell's results for the GMM framework.

Following the decomposition in equation (7), the sequence of alternatives can be rewritten as:

$$
\Omega^{-1 / 2} E g\left(x_{T t}, \theta_{0}\right)=P_{G} \Omega^{-1 / 2} \frac{h\left(\eta, \tau, \frac{t}{T}\right)}{\sqrt{T}}+\left(I_{q}-P_{G}\right) \Omega^{-1 / 2} \frac{h\left(\eta, \tau, \frac{t}{T}\right)}{\sqrt{T}}
$$

where the first component is the local alternative on the identifying moments and the second is the local alternative on the overidentifying restrictions.

For instability in the parameter vector, consider a general local alternative of the form (see Sowell $(1996 \mathrm{a}))$

$$
\beta_{T t}=\beta_{0}+\frac{f\left(\eta, \tau, \frac{t}{T}\right)}{\sqrt{T}}
$$

for $t=1, \ldots, T$. A Taylor expansion of $g\left(x_{T t}, \theta_{T t}\right)$ yields

$$
E g\left(x_{T t}, \beta_{0}\right)=-G_{\beta} \frac{f\left(\eta, \tau, \frac{t}{T}\right)}{\sqrt{T}}+o_{p}(1) .
$$

If we substitute this expression into (9), the expression above is orthogonal to the second component of (9) and therefore puts restrictions on the first component (the identifying restrictions). In the case of pure structural $P_{G}=P_{G_{\beta}}=\Omega^{-1 / 2} G_{\beta}\left[G_{\beta}^{\prime} \Omega^{-1} G_{\beta}\right]^{-1} G_{\beta}^{\prime} \Omega^{-1 / 2}$. The alternative that at some point there is a single structural break at $\tau, H_{A}^{I}(\tau)$, is represented as:

$$
\beta_{T t}= \begin{cases}\beta_{0} & t=1, \ldots,[T \tau] \\ \beta_{0}+\frac{\eta}{\sqrt{T}} & t=[T \tau]+1, \ldots, T\end{cases}
$$

which corresponds to a specific form for $f\left(\eta, \tau, \frac{t}{T}\right)$.

For instability of the overidentifying restrictions at a single breakpoint $\tau$ occurring before and/or after the breakpoint, this is formally stated as $H_{A}^{O}(\tau)=H_{A}^{O 1}(\tau) \cap H_{A}^{O 2}(\tau)$ with:

$$
\begin{array}{lll}
H_{A}^{O 1}(\tau):\left(I_{q}-P_{G}\right) \Omega^{-1 / 2} E\left[g\left(x_{t}, \theta_{0}\right)\right] & =\frac{\eta_{1}}{\sqrt{T}} & \forall t=1, \ldots,[T \tau] \\
H_{A}^{O 2}(\tau):\left(I_{q}-P_{G}\right) \Omega^{-1 / 2} E\left[g\left(x_{t}, \theta_{0}\right)\right]=\frac{\eta_{2}}{\sqrt{T}} & \forall t=[T \tau]+1, \ldots, T .
\end{array}
$$

and $\eta_{1} \neq \eta_{2} \cdot{ }^{3}$ This formulation of the alternative for a single breakpoint corresponds to a specific form of $h\left(\eta, \tau, \frac{t}{T}\right)$ in (9). 


\section{Tests for a structural change based on implied probabilities}

Ramalho and Smith (2005) introduced in an i.i.d. setting a specification test for moment conditions based on implied probabilities similar in spirit to the classical Pearson chi-square goodness-of-fit test. The test is based on the following statistic:

$$
\sum_{t=1}^{T}\left(T \pi_{t}^{G E L}\left(\tilde{\theta}_{T}\right)-1\right)^{2}
$$

They showed that such statistic is asymptotically equivalent to the overidentifying moment restrictions J-test proposed by Hansen (1982). Guay and Pelgrin (2007) and Guggenberger, Ramalho and Smith (2007) also used this statistic in the time series context and showed that:

$$
\frac{1}{2 K+1} \sum_{t=1}^{T}\left(T \pi_{t}\left(\tilde{\theta}_{T}\right)-1\right)^{2}
$$

is asymptotically first-order equivalent to the overidentifying moment restrictions J-test. However, as shown by Ghysels and Hall (1990), the J-test has no power to detect structural change in parameter values, a property that is shared by the specification tests, (11), and the one just above, as we demonstrate below.

In the same spirit, we first consider a test statistic based on the partial-sample implied probabilities evaluated at the restricted estimator for GEL. The implied probabilities structural change (IPSC) test statistic proposed to detect instability is given by the following partial sum:

$$
\operatorname{IPSC}_{T}^{G E L}(s)=\frac{s}{2 K_{T}+1} \sum_{t=1}^{[T s]}\left(T \pi_{t}^{G E L}\left(\tilde{\theta}_{T}, s\right)-1\right)^{2}
$$

with

$$
\pi_{t}^{G E L}\left(\tilde{\theta}_{T}, s\right)=\frac{\rho_{1}\left(\tilde{\gamma}_{1 T}(s)^{\prime} g_{t T}\left(\tilde{\theta}_{T}, s\right)\right)}{\sum_{t=1}^{T} \rho_{1}\left(\tilde{\gamma}_{T}(s)^{\prime} g_{t T}\left(\tilde{\theta}_{T}, s\right)\right)},
$$

where for the numerator $\tilde{\gamma}_{1 T}(s)$ is the solution of the following maximization problem:

$$
\gamma_{1 T}(\beta, \delta)=\arg \sup _{\gamma_{1} \in \hat{\Gamma}_{T}(\beta, \delta)} \sum_{t=1}^{[T s]} \frac{\left[\rho\left(\gamma_{1}(\beta, \delta)^{\prime} g_{t T}(\beta, \delta)\right)-\rho_{0}\right]}{T}
$$

evaluated at the restricted estimator $\tilde{\theta}_{T}$ and for the denominator $\tilde{\gamma}_{T}(s)=\left(\tilde{\gamma}_{1 T}(s)^{\prime}, \tilde{\gamma}_{2 T}(s)^{\prime}\right)^{\prime}$ with $\tilde{\gamma}_{1 T}(s)$ defined as above and

$$
\gamma_{2 T}(\beta, \delta)=\arg \sup _{\gamma_{2} \in \hat{\Gamma}_{T}(\beta, \delta)} \sum_{t=[T s]+1}^{T} \frac{\left[\rho\left(\gamma_{2}(\beta, \delta)^{\prime} g_{t T}(\beta, \delta)\right)-\rho_{0}\right]}{T} .
$$

It is crucial to note that this statistic is based on the unrestricted implied probabilities evaluated at the restricted estimator of $\theta$.

The next Theorem establishes the asymptotic distribution for this general test of a structural change under the null and the sequence of alternatives defined in (8). 
Theorem 4.1. Under Assumptions (7.1) to (7.12), the following processes indexed by s for $s \in[0,1]$ satisfy, under the null (6),

$$
\operatorname{IPSC}_{T}^{G E L}(s) \Rightarrow B B_{p}(s)^{\prime} B B_{p}(s)+B_{(q-p)}^{\prime}(s) B_{(q-p)}(s)
$$

and under the alternative (8)

$$
\begin{aligned}
\operatorname{IPSC}_{T}^{G E L}(s) \Rightarrow & B B_{p}(s)^{\prime} B B_{p}(s)+(H(s)-s H(1))^{\prime} \Omega^{-1 / 2} P_{G} \Omega^{-1 / 2}(H(s)-s H(1)) \\
& +B_{q-p}(s)^{\prime} B_{q-p}(s)+H(s)^{\prime} \Omega^{-1 / 2}\left(I-P_{G}\right) \Omega^{-1 / 2} H(s)
\end{aligned}
$$

where $B_{(q-p)}(s)$ is a $(q-p)$-vector of standard Brownian motion, $B B_{p}(s)=B_{p}(s)-s B_{p}(1)$ is a p-vector of Brownian bridge with $p=r+\nu$ and $H(s)=\int_{0}^{s} h(\eta, \tau, r) d r$.

Proof: See the Appendix.

The Theorem shows that the structural change test based on this quadratic form of the partialsample sum of the implied probabilities evaluated at the full-sample estimator combines two components. The first component of the limiting distribution, which is a function of Brownian bridges, corresponds to a stability test for the whole set of parameters $(\beta$ and $\delta)$ and the second component to a stability test for the overidentifying restrictions. This test statistic, based on implied probabilities, can be viewed as a more general form of misspecification due to instability than just a test for parameter variation. The predictive tests proposed by Ghysels, Guay and Hall (1997) shares the same properties. In the Appendix we show that the IPSC test is asymptotically equivalent to the test statistic proposed by Sowell (1996b). He showed that his test statistic is optimal for a one time jump in all moment conditions where the location of the jump is unknown and consistent for arbitrary alternatives. These properties are then shared by our test. Note that the limiting distribution exists for $s=0$ which is trivially equal to 0 . For $s=1$, the test statistic corresponds to the specification test for moment conditions developed by Guay and Pelgrin (2007) and Guggenberger et al. (2007) and by the above Theorem the limiting distribution is given by:

$$
B_{q-p}(1)^{\prime} B_{q-p}(1)+H(1)^{\prime} \Omega^{-1 / 2}\left(I-P_{G}\right) \Omega^{-1 / 2} H(1) .
$$

This limiting distribution shows that this test statistic has a chi-square distribution with $q-p$ degrees of freedom under the null and has local power equal to the size to detect instability in parameter values, as the J-test proposed by Hansen (1982). Moreover, the test statistic (11) can not detect asymptotically instability in the overidentifying restrictions for which $\left(I-P_{G}\right) \Omega^{-1 / 2} H(1)=0$.

When the breakpoint is unknown, one can construct statistics across $s \in S$. In the context of maximum likelihood estimation, Andrews and Ploberger (1994) derive asymptotic optimal tests for a gaussian a priori of the amplitude of the structural change based on the Neyman-Pearson approach which are characterized by an average exponential form. The Sowell (1996a) optimal tests are a 
generalization of the Andrews and Ploberger approach to the case of two measures that do not admit densities. The most powerful test is given by the Radon-Nikodym derivative of the probability measure implied by the local alternative with respect to the probability measure implied by the null hypothesis.

The optimal average exponential form applied to a statistic $Q_{T}(s)$ for $s \in S$ has the following form:

$$
\operatorname{Exp}-Q_{T}=(1+c)^{-q / 2} \int \exp \left(\frac{1}{2} \frac{c}{1+c} Q_{T}(s)\right) d J(s)
$$

where various choices of $c$ determine power against close or more distant alternatives and $J(\cdot)$ is the weight function over the value of $s \in S$. In the case of close alternatives $(c=0)$, the optimal test statistic takes the average form, ave $Q_{T}=\int_{S} Q_{T}(s) d J(s)$. For a distant alternative $(c=\infty)$, the optimal test statistics takes the exponential form, $\exp Q_{T}=\log \left(\int_{S} \exp \left[\frac{1}{2} Q_{T}(s)\right] d J(s)\right)$. The supremum form often used in the literature corresponds to the case where $\frac{c}{(1+c)} \rightarrow \infty$. The sup test is given by $\sup Q_{T}=\sup _{s \in S} Q_{T}(s)$.

The following Theorem gives the asymptotic distribution for the average exponential mapping for $Q_{T}^{I P S C}(s)$ where $Q_{T}^{I P S C}(s)$ corresponds to the statistic presented above based on the implied probabilities.

Theorem 4.2. Under the null hypothesis $H_{0}$ in (6) and Assumptions 7.1 to \%.12, the following processes indexed by $s$ for a given set $S$ whose closure lies in [0,1] satisfy:

$\sup Q_{T}^{I P S C} \Rightarrow \sup _{s \in S} Q_{p, q-p}(s), \quad \operatorname{ave} Q_{T}^{I P S C} \Rightarrow \int_{S} Q_{p, q-p}(s) d J(s), \quad \exp Q_{T}^{I P S C} \Rightarrow \log \left(\int_{S} \exp \left[\frac{1}{2} Q_{p, q-p}(s)\right] d J(s)\right)$, with

$$
Q_{p, q-p}(s)=B B_{p}(s)^{\prime} B B_{p}(s)+B_{(q-p)}(s)^{\prime} B_{(q-p)}(s)
$$

and $J(s)$ is the weighting distribution function for the location of the instability s.

This result is obtained through the application of the continuous mapping theorem (see Pollard (1984)). The asymptotic critical values were obtained using simulated Brownian motions and Brownian bridges over 10,000 replications for maximum values of $p$ and $q-p$ set at 10 . The critical values appear in Tables 1 to 3 for symmetric intervals $S=\left[s_{0}, 1-s_{0}\right]$. Critical values for the entire sample appear at $s_{0}=0$.

An asymptotically equivalent modified statistic to (12) in the spirit of the Neyman-modified chisquare test is given by:

$$
\operatorname{IPSCM} M_{T}^{G E L}(s)=\frac{s}{2 K_{T}+1} \sum_{t=1}^{[T s]} \frac{\left(T \pi_{t}^{G E L}\left(\tilde{\theta}_{T}, s\right)-1\right)^{2}}{T \pi_{t}^{G E L}\left(\tilde{\theta}_{T}, s\right)}
$$


since $T \pi_{t}^{G E L}\left(\tilde{\theta}_{T}, s\right)=1+o_{p}(1)$ under the null.

In the sequel, we propose structural change tests based on implied probabilities specially designed to detect instability in the parameters of interest or in the overidentifying restrictions.

\subsection{Tests for a structural change in the parameters based on implied probabili-} ties

The test statistics proposed to specifically detect parameter instability are based on the difference between the partial sum of unrestricted implied probabilities evaluated at the unrestricted estimator $\hat{\theta}_{T}(s)$ and the partial sum of unrestricted implied probability evaluated at the restricted estimator $\tilde{\theta}_{T}$. More precisely the test statistic is defined as:

$$
\operatorname{IPSC}_{T}^{I, G E L}(s)=\frac{1}{2 K_{T}+1} \sum_{t=1}^{T}\left(T \pi_{t}^{G E L}\left(\hat{\theta}_{T}(s), s\right)-T \pi_{t}^{G E L}\left(\tilde{\theta}_{T}, s\right)\right)^{2}
$$

with

$$
\pi_{t}^{G E L}\left(\tilde{\theta}_{T}, s\right)=\frac{\rho_{1}\left(\tilde{\gamma}_{T}(s)^{\prime} g_{t T}\left(\tilde{\theta}_{T}, s\right)\right)}{\sum_{t=1}^{T} \rho_{1}\left(\tilde{\gamma}_{T}(s)^{\prime} g_{t T}\left(\tilde{\theta}_{T}, s\right)\right)}
$$

where $\tilde{\gamma}_{T}(s)=\left(\tilde{\gamma}_{1 T}(s)^{\prime} \tilde{\gamma}_{2}(s)^{\prime}\right)^{\prime}$ is the solution of the respective maximization problems defined in equations (13) and (14) and $\pi_{t}^{G E L}\left(\hat{\theta}_{T}(s), s\right)$ is defined in equation (5).

The next Theorem establishes the asymptotic distribution for this test of structural change in the parameter values under the null that the vector $\beta$ is constant throughout the sample.

Theorem 4.3. Under the null hypothesis $H_{0}$ in (3) and Assumptions (7.1) to (7.12), the following processes indexed by $s$ for a given set $S$ whose closure lies in $(0,1)$ satisfy:

$$
\operatorname{IPSC}_{T}^{I, G E L}(s) \Rightarrow Q_{r}(s)=\frac{B B_{r}(s)^{\prime} B B_{r}(s)}{s(1-s)}
$$

and under the alternative (8)

$$
Q_{r}(s)=\frac{B B_{r}(s)^{\prime} B B_{r}(s)}{s(1-s)}+\frac{(H(s)-s H(1))^{\prime} \Omega^{-1 / 2} P_{G_{\beta}} \Omega^{-1 / 2}(H(s)-s H(1))}{s(1-s)},
$$

where $B B_{r}(s)=B_{r}(s)-s B_{r}(1)$ is a Brownian bridge, $B_{r}$ is r-vector of independent Brownian motions and $P_{G_{\beta}}=\Omega^{-1 / 2} G_{\beta}\left[\left(G_{\beta}\right)^{\prime} \Omega^{-1} G_{\beta}\right]^{-1}\left(G_{\beta}\right)^{\prime} \Omega^{-1 / 2}$. Moreover,

$$
\begin{aligned}
\sup I P S C_{T}^{I, G E L} \Rightarrow & \sup _{s \in S} Q_{r}(s), \quad \text { aveIPSC } C_{T}^{I, G E L} \Rightarrow \int_{S} Q_{r}(s) d J(s), \\
& \exp I P S C_{T}^{I, G E L} \Rightarrow \log \left(\int_{S} \exp \left[\frac{1}{2} Q_{r}(s)\right] d J(s)\right) .
\end{aligned}
$$


Proof: See the Appendix.

The Theorem shows that the asymptotic distribution of the test based on implied probabilities is asymptotically equivalent under the null and the alternative to the $W$ ald, $L M$ and $L R$ tests for parameter instability (see Andrews, 1993). More precisely, the limiting distribution is function of a $r$-vector of Brownian bridge with the same dimension as the parameter vector $\beta$. However, the small sample properties of tests based on implied probabilities can differ to those of more standard tests. Note that, in contrast to Theorem 4.2, the limiting distribution in Theorem 4.3 is valid only for $S$ in the open interval $(0,1)$.

The two following modified statistics are asymptotically equivalent to the one defined above:

$$
\operatorname{IPSCM} 1_{T}^{I, G E L}(s)=\frac{1}{2 K_{T}+1} \sum_{t=1}^{T} \frac{\left(T \pi_{t}^{G E L}\left(\hat{\theta}_{T}(s), s\right)-T \pi_{t}^{G E L}\left(\tilde{\theta}_{T}, s\right)\right)^{2}}{T \pi_{t}^{G E L}\left(\hat{\theta}_{T}(s), s\right)}
$$

and

$$
\operatorname{IPSCM} 2_{T}^{I, G E L}(s)=\frac{1}{2 K_{T}+1} \sum_{t=1}^{T} \frac{\left(T \pi_{t}^{G E L}\left(\hat{\theta}_{T}(s), s\right)-T \pi_{t}^{G E L}\left(\tilde{\theta}_{T}, s\right)\right)^{2}}{T \pi_{t}^{G E L}\left(\tilde{\theta}_{T}, s\right)}
$$

\subsection{Tests for a structural change in the overidentifying restrictions based on implied probabilities}

Now, we propose a test statistic based on implied probabilities designed specially to detect instability in the overidentifying restrictions. The statistic is powerful against violation of $H_{0}^{O 1}(s)$ and $H_{0}^{O 2}(s)$ and is asymptotically equivalent to the ones proposed by Hall and Sen (1999) and thus shares its asymptotic properties, but perhaps not its small sample properties. As previously, the sample is split in two subamples with a single breakpoint at $[T s]$. An estimator of the parameter vector is obtained with the first subsample (for $t=1, \ldots,[T s]$ ) and with the second subsample (for $t=[T s]+1, \ldots, T$ ). The entire parameter vector is allowed to vary for both subsamples. The proposed statistic designed to detect instability in the overidentifying restrictions is based on the specification test for moment conditions given in equation (11) for the first and second subsamples.

More precisely the statistic is defined as:

$$
\begin{aligned}
\operatorname{IPSC}_{T}^{O, G E L}(s)= & \frac{1}{2 K_{T}+1} \sum_{t=1}^{[T s]}\left([T s] \pi_{t}^{G E L}\left(\hat{\theta}_{1 T}(s), s\right)-1\right)^{2} \\
& +\frac{1}{2 K_{T}+1} \sum_{t=[T s]+1}^{T}\left((T-[T s]) \pi_{t}^{G E L}\left(\hat{\theta}_{2 T}(s), s\right)-1\right)^{2}
\end{aligned}
$$

where $\hat{\theta}_{1 T}(s)=\hat{\beta}_{1 T}(s)$ and $\hat{\theta}_{2 T}(s)=\hat{\beta}_{2 T}(s)$. The statistic is the sum of the overidentifying restrictions statistics (11) but for the first and the second parts of the sample evaluated at the unrestricted estimator. 
The next Theorem establishes the asymptotic distribution of this statistic and the corresponding mappings.

Theorem 4.4. Under Assumptions (7.1) to (7.12), the following processes indexed by $s$ for a given set $S$ whose closure lies in $(0,1)$ satisfy

$$
\operatorname{IPSC}_{T}^{O, G E L}(s) \Rightarrow Q_{q-r}(s)
$$

with under the null of no structural change

$$
Q_{q-r}(s) \Rightarrow \frac{B_{q-r}(s)^{\prime} B_{q-r}(s)}{s}+\frac{\left[B_{q-r}(1)-B_{q-r}(s)\right]^{\prime}\left[B_{q-r}(1)-B_{q-r}(s)\right]}{1-s}
$$

and under the alternative (8)

$$
\begin{aligned}
Q_{q-r}(s) \Rightarrow & \frac{B_{q-r}(s)^{\prime} B_{q-r}(s)}{s}+\frac{H(s)^{\prime} \Omega^{-1 / 2}\left(I-P_{G}\right) \Omega^{-1 / 2} H(s)}{s} \\
& \frac{\left[B_{q-r}(1)-B_{q-r}(s)\right]^{\prime}\left[B_{q-r}(1)-B_{q-r}(s)\right]}{1-s}+\frac{[H(1)-H(s)]^{\prime} \Omega^{-1 / 2}\left(I-P_{G}\right) \Omega^{-1 / 2}[H(1)-H(s)]}{(1-s)}
\end{aligned}
$$

where $B_{q-r}(s)$ is a $q-r$-vector of standard Brownian motion and $P_{G}=P_{G_{\beta}}$ with $P_{G_{\beta}}=$ $\Omega^{-1 / 2} G_{\beta}\left(G_{\beta}^{\prime} \Omega^{-1} G_{\beta}\right)^{-1} G^{\prime} \Omega^{-1 / 2}$. Moreover,

$$
\begin{aligned}
& \sup I P S C_{T}^{O, G E L} \Rightarrow \sup _{s \in S} Q_{q-r}(s), \quad \text { aveIPSC } C_{T}^{O, G E L} \Rightarrow \int_{S} Q_{q-r}(s) d J(s)
\end{aligned}
$$

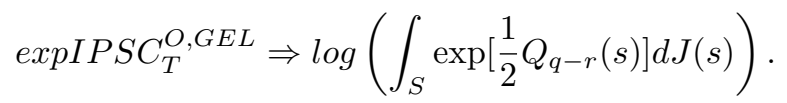

Proof: See the Appendix.

The Theorem shows that the proposed test statistics can detect instability occurring in overidentifying restrictions before and after the breakpoint. Indeed, the term $\frac{H(s)^{\prime} \Omega^{-1 / 2}\left(I-P_{G}\right) \Omega^{-1 / 2} H(s)}{s}$ corresponds to a structural change in the moment conditions before the breakpoint $s$ while the term $\frac{[H(1)-H(s)]^{\prime} \Omega^{-1 / 2}\left(I-P_{G}\right) \Omega^{-1 / 2}[H(1)-H(s)]}{(1-s)}$ to a structural change after the breakpoint $s$.

The asymptotic critical values for the interval $S=[.15, .85]$ can be found in Hall and Sen (1999). For other symmetric interval $\left[s_{0}, 1-s_{0}\right]$, critical values can be obtained in Guay (2003), Tables 1 to 3 for a number of overidentifying restrictions divided by 2 (in those tables). To see this, note that the critical values for the supremum, the average and the log exponential mappings applied to $\frac{B_{2 q-2 r}(s)^{\prime} B_{2 q-2 r}(s)}{s}$ are equivalent to ones corresponding to $\frac{B_{q-r}(s)^{\prime} B_{q-r}(s)}{s}+\frac{\left(B_{q-r}(1)-B_{q-r}(s)\right)^{\prime}\left(B_{q-r}(1)-B_{q-r}(s)\right)}{1-s}$ for a symmetric interval $S^{4}$

An asymptotically equivalent statistic to $\operatorname{IPSC}_{T}^{O, G E L}(s)$ in the spirit of the Neyman-modified 
chi-square test is given by:

$$
\begin{aligned}
\operatorname{IPSCM}_{T}^{O, G E L}(s)= & \frac{1}{2 K_{T}+1} \sum_{t=1}^{[T s]} \frac{\left([T s] \pi_{t}^{G E L}\left(\hat{\theta}_{1 T}(s), s\right)-1\right)^{2}}{[T s] \pi_{t}^{G E L}\left(\hat{\theta}_{1 T}(s), s\right)} \\
& +\frac{1}{2 K_{T}+1} \sum_{t=[T s]+1}^{T} \frac{\left((T-[T s]) \pi_{t}^{G E L}\left(\hat{\theta}_{2 T}(s), s\right)-1\right)^{2}}{(T-[T s]) \pi_{t}^{G E L}\left(\hat{\theta}_{2 T}(s), s\right)} .
\end{aligned}
$$

\subsection{A structural change test robust to weak identification or cases in which parameters are completely unidentified}

We propose here a test statistic robust to the context of weak identification, as defined by Stock and Wright (2000), or robust to cases in which parameters are completely unidentified. Consider the pure structural change case with $\theta=\left(\beta^{\prime}, \beta^{\prime}\right)^{\prime}$. We need to introduce a restricted estimator $\tilde{\theta}_{T}(s)$ obtained with the partial-sample GEL objective function. A restricted partial-sample General Empirical Likelihood estimator $\left\{\tilde{\theta}_{T}(s)\right\}$ is a sequence of random vectors such that:

$$
\begin{aligned}
\tilde{\theta}_{T}(s) & =\arg \min _{\theta \in \Theta} \sup _{\gamma(s) \in \tilde{\Gamma}_{T}(\theta, s)} \sum_{t=1}^{T} \frac{\left[\rho\left(k \gamma(s)^{\prime} g_{t T}(\theta, s)\right)-\rho_{0}\right]}{T} \\
& =\arg \min _{\beta \in B} \sup _{\gamma(s) \in \tilde{\Gamma}_{T}(\theta, s)}\left(\sum_{t=1}^{[T s]} \frac{\left[\rho\left(k \gamma_{1}^{\prime} g_{t T}(\beta)\right)-\rho_{0}\right]}{T}+\sum_{t=[T s]+1}^{T} \frac{\left[\rho\left(k \gamma_{2}^{\prime} g_{t T}(\beta)\right)-\rho_{0}\right]}{T}\right)
\end{aligned}
$$

for all $s \in S$ where $g_{t T}(\theta, s)=\left(g_{t T}(\beta)^{\prime}, 0^{\prime}\right)^{\prime} \in R^{2 q \times 1}$ for $t=1, \ldots,[T s]$ and $g_{t T}(\theta, s)=\left(0^{\prime}, g_{t T}(\beta)^{\prime}\right)^{\prime} \in$ $R^{2 q \times 1}$ for $t=[T s]+1, \ldots, T$ with $\gamma(s)=\left(\gamma_{1}(s)^{\prime}, \gamma_{2}(s)^{\prime}\right)^{\prime} \in R^{2 q \times 1}$ and $\tilde{\Gamma}_{T}(\theta, s)=\{\gamma(s)=$ $\left.\left(\gamma_{1}(s)^{\prime}, \gamma_{2}(s)^{\prime}\right)^{\prime}: k \gamma(s)^{\prime} g_{t T}(\theta, s)\right\}$

The statistic is defined as:

$$
\begin{aligned}
\operatorname{IPSC}_{T}^{R, G E L}(s)= & \frac{1}{2 K_{T}+1} \sum_{t=1}^{[T s]}\left([T s] \pi_{t}^{G E L}\left(\tilde{\theta}_{T}(s), s\right)-1\right)^{2} \\
& +\frac{1}{2 K_{T}+1} \sum_{t=[T s]+1}^{T}\left((T-[T s]) \pi_{t}^{G E L}\left(\tilde{\theta}_{T}(s), s\right)-1\right)^{2} .
\end{aligned}
$$

The statistic depends on unrestricted implied probabilities for the first and the second parts of the sample evaluated at the restricted partial-sample GEL estimators.

We show in the Appendix that this test statistic is asymptotically equivalent at first-order to the $S$-based test in Caner (2007). The test statistic is not asymptotically pivotal but asymptotically boundedly pivotal. The bound is then free of nuisance parameters and robust to identification problems under the null. The following theorem gives this asymptotic bound under the null of no structural change and the local alternative (8). 
Theorem 4.5. Under Assumptions (7.1) to (7.12), the following processes indexed by sor a given set $S$ whose closure lies in $(0,1)$ satisfy

$$
\operatorname{IPSC}_{T}^{R, G E L}(s) \Rightarrow Q_{q}(s)
$$

are asymptotically boundedly pivotal and the asymptotic bound distribution is given by:

$$
Q_{q}(s) \Rightarrow \frac{B_{q}(s)^{\prime} B_{q}(s)}{s}+\frac{\left[B_{q}(1)-B_{q}(s)\right]^{\prime}\left[B_{q}(1)-B_{q}(s)\right]}{1-s}
$$

under the null of no structural change and under the alternative (8) by

$$
\begin{aligned}
Q_{q}(s) \Rightarrow & \frac{B_{q}(s)^{\prime} B_{q}(s)}{s}+\frac{H(s)^{\prime} \Omega^{-1} H(s)}{s} \\
& \frac{\left[B_{q}(1)-B_{q}(s)\right]^{\prime}\left[B_{q}(1)-B_{q}(s)\right]}{1-s}+\frac{[H(1)-H(s)]^{\prime} \Omega^{-1}[H(1)-H(s)]}{(1-s)},
\end{aligned}
$$

where $B_{q}(s)$ is a q-vector of standard Brownian motion.

Proof: See the Appendix.

The asymptotic bounds are valid for strong, weakly identified and completely unidentified cases. Critical values under the null for the different mappings are given in the same tables as those of subsection 4.2. The asymptotic bound under the local alternative allows us to examine the power of the test under different assumptions with respect to identification. Consider the decomposition of the alternative according to (9), namely

$$
\frac{h\left(\eta, \tau, \frac{t}{T}\right)}{\sqrt{T}}=P_{G_{\beta}} \Omega^{-1 / 2} \frac{h\left(\eta, \tau, \frac{t}{T}\right)}{\sqrt{T}}+\left(I_{q}-P_{G_{\beta}}\right) \Omega^{-1 / 2} \frac{h\left(\eta, \tau, \frac{t}{T}\right)}{\sqrt{T}} .
$$

This decomposition and the asymptotic bound under the alternative show that the ability of the test statistic to detect a structural change in the parameter values depends on the Jacobian matrix $G_{\beta}$ (see equation 10). Under weak identification, $G_{\beta}$ has a weak value which means that $G_{\beta, T}\left(\theta_{0}\right)=\frac{C}{\sqrt{T}}$ for a $C$ matrix of dimension $q \times p$. In this case, the test statistic has trivial power equals to the size. Obviously, it is also the case when the parameters are unidentified because $G=0$. In fact, the test statistic will detect instability in parameter values for alternatives such that $\frac{h\left(\eta, \tau, \frac{t}{T}\right)}{T^{\alpha}}$ for $\alpha \geq 1$ in the weak identification (again see Stock and Wright (2000)). For instance, the test statistic will detect structural change in the parameter values with no trivial power for the following fixed alternative:

$$
H_{A}^{I}(s)=\left\{\begin{array}{lll}
\beta_{T t}=\beta_{0} & \forall t=1, \ldots,[T s] \\
\beta_{T t}=\beta_{0}+\eta & \forall t=[T s]+1, \ldots, T .
\end{array}\right.
$$

The discussion above also holds for the $S$-based test statistic proposed by Caner (2007) who derived the bound only under the null. 


\section{Simulation evidence}

To evaluate the performance of the test statistics, and to facilitate comparison with previous studies, we use the data generating process found in Ghysels et al. (1997) and in Hall and Sen (1999). The time series model used is an $\operatorname{AR}(1)$ process for the variable $x_{t}$. One parameter is estimated, the autoregressive parameter (denoted by $\theta$ in the expression below), using two moment conditions formed with the lagged values of $x_{t}$.

The data generating process is given by

$$
x_{t}=\theta_{i} x_{t-1}+u_{t}
$$

for $t=1, \ldots, T$. Structural change in the identifying restrictions (in the parameter) is studied by considering different values of $\theta_{i}$ where the index $i=1,2$ denotes the first or second subsamples. Structural change in the overidentifying restrictions is studied by allowing for an ARMA(1,2) model

$$
x_{t}=\theta_{i} x_{t-1}+u_{t}+\alpha u_{t-2}
$$

and considering nonzero values of $\alpha$ in the second subsample. The break is set at $T / 2$. In the above, $u_{t} \sim N(0,1)$. The sample size was set to 200 observations and the number of Monte Carlo replications was 2000 (for each replication one constrained and 242 unconstrained optimization problems were solved for a total of 486,000 numerical optimization).

Table 4 summarizes the different parameterizations. The null hypothesis of structural stability is denoted by $H_{0}^{S S}$ (DGP 1 to 3 ). For those DGPs we vary the magnitude of the autoregressive parameter $\theta$. The alternatives of instability in the parameters or in the overidentifying restrictions

are denoted by $H_{A}^{I}$ (DGP 4 to 6 ), where we vary the magnitude of the change in the autoregressive parameter, and $H_{A}^{O}$ (DGP 7 to 10) where we consider various values of the moving average parameter, respectively. In this situation only one parameter is estimated using two moment conditions created with the first two lags of $x_{t}$. Under $H_{0}^{S S}$, where $\alpha=0$, the instruments are appropriate. Under the first class of alternative hypothesis $\left(H_{A}^{I}\right)$ the two instruments are also valid while they no longer are for the second part of the sample with the second class of alternative hypothesis $\left(H_{A}^{O}\right)$.

Smoothing the moment conditions can be done via an appropriate choice of $K_{T}$. In a GMM setting this is equivalent to using some form of estimate of the long-run covariance matrix of the moment conditions (for example using the Newey-West estimator as the weight matrix in quadratic form). Most of the previous simulation work considered a fixed degree of smoothing (see for example Gregory et al. (2002) and Guggenberger and Smith (2008)). Otsu (2006) did look at fixed smoothing but also looked at applying the automatic bandwidth selection rule of Newey and West (1994).

Our Monte Carlo study is not particularly designed to investigate smoothing because under the null hypothesis the optimal value of $K_{T}$ is 0 while it is not 0 under the alternative hypothesis $H_{A}^{O}$. 
For this reason we set $K_{T}=0$ for all DGPs except DGPs 7, 8, 9 and 10. For these DGPs we select a bandwidth parameter chosen via the automatic, data-driven, procedure which chooses a bandwidth $m_{T}$ which is then transformed as $K_{T}=\left[\left(m_{T}-1\right) / 2\right]$. The average, taken over Monte Carlo replications, $K_{T}$ was found to vary between 1.6 and 2.3, increasing with the moving average component. A complete analysis of the effects of smoothing is left for future work. Lastly, a trimming rule of 0.15 was used, namely $S=[.15, .85]$.

Table 5 contains the results for the general specification tests IPSC and IPSCM (the supremum, exponential and average version of the test statistics are presented). Table 6 contains the rejection frequencies for the test statistics designed to have power against a structural change in the parameters while Table 7 presents the results for test statistics which are designed to have power against a structural change in the overidentifying restrictions. All test statistics were computed in the GEL setting using exponential tilting. The tests used for comparison appear in Guay and Lamarche (2009). For completeness we report the Wald, LM, LR and O tests (all computed in a GEL environment) below:

$$
\operatorname{Wald}_{T}(s)=T\left(\hat{\beta}_{1 T}(s)-\hat{\beta}_{2 T}(s)\right)^{\prime}\left(\hat{V}_{\Omega}(s)\right)^{-1}\left(\hat{\beta}_{1 T}(s)-\hat{\beta}_{2 T}(s)\right)
$$

where $\hat{V}_{\Omega}(s)=\left(\hat{V}_{1}(s) / s+\hat{V}_{2}(s) /(1-s)\right)$ and $\hat{V}_{i}(s)=\left(\hat{G}_{i, t T}^{\beta}(s)^{\prime} \hat{\Omega}_{i, T}^{-1}(s) \hat{G}_{i}^{\beta}(s)\right)^{-1}$ for $i=1,2$ corresponding to the first and the second part of the sample. For the first part of the sample:

$$
\begin{aligned}
\hat{G}_{1, t T}^{\beta}(s) & =\frac{1}{[T s]} \sum_{t=1}^{[T s]} \frac{\partial g_{t T}\left(\hat{\beta}_{1}(s), \hat{\delta}(s)\right)}{\partial \beta_{1}^{\prime}} \\
\widehat{\Omega}_{1 T}(s) & =\frac{2 K_{T}+1}{[T s]} \sum_{t=1}^{[T s]} g_{t T}\left(\beta_{1}(s), \delta\right) g_{t T}\left(\hat{\beta}_{1}(s), \hat{\delta}(s)\right)^{\prime}
\end{aligned}
$$

and for the second part of the sample:

$$
\begin{aligned}
\widehat{G}_{2, t T}^{\beta} & =\frac{1}{T-[T s]} \sum_{t=[T s]+1}^{T} \frac{\partial g_{t T}\left(\hat{\beta}_{2}(s), \hat{\delta}(s)\right)}{\partial \beta_{2}^{\prime}} \\
\widehat{\Omega}_{2 T}(s) & =\frac{2 K_{T}+1}{T-[T s]} \sum_{t=[T s]+1}^{T} g_{t T}\left(\hat{\beta}_{2}(s), \hat{\delta}(s)\right) g_{t T}\left(\hat{\beta}_{2}(s), \hat{\delta}(s)\right)^{\prime} .
\end{aligned}
$$

The Lagrange Multiplier statistic is given by:

$$
L M_{T}(s)=\frac{T}{s(1-s)} g_{1 T}\left(\tilde{\theta}_{T}, s\right)^{\prime} \hat{\Omega}_{T}^{-1} \hat{G}_{t T}^{\beta}\left[\left(\hat{G}_{t T}^{\beta}\right)^{\prime} \hat{\Omega}_{T}^{-1} \hat{G}_{t T}^{\beta}\right]^{-1}\left(\hat{G}_{t T}^{\beta}\right)^{\prime} \hat{\Omega}_{T}^{-1} g_{1 T}\left(\tilde{\theta}_{T}, s\right) .
$$


where

$$
\begin{aligned}
g_{1 T}\left(\tilde{\theta}_{T}, s\right) & =\frac{1}{T} \sum_{t=1}^{[T s]} g_{t T}\left(\tilde{\theta}_{T}\right), \\
\hat{G}_{t T}^{\beta} & =\frac{1}{T} \sum_{t=1}^{T} \frac{\partial g_{t T}(\tilde{\beta}, \tilde{\delta})}{\partial \beta^{\prime}}, \\
\widehat{\Omega}_{T} & =\frac{2 K_{T}+1}{T} \sum_{t=1}^{T} g_{t T}(\tilde{\beta}, \tilde{\delta}) g_{t T}(\tilde{\beta}, \tilde{\delta})^{\prime} .
\end{aligned}
$$

The LR-like statistic is defined as:

$$
L R_{T}(s)=\frac{2 T}{2 K+1}\left[\sum_{t=1}^{T} \frac{\left[\rho\left(\tilde{\gamma}(s)^{\prime} g_{t T}(\tilde{\theta}, s)\right)-\rho_{0}\right]}{T}-\sum_{t=1}^{T} \frac{\left[\rho\left(\hat{\gamma}(s)^{\prime} g_{t T}(\hat{\theta}(s), s)\right)-\rho_{0}\right]}{T}\right]
$$

And finally, the test statistic proposed by Hall and Sen (1999) is

$$
O_{T}(s)=O 1_{T}(s)+O 2_{T}(s)
$$

where

$$
O 1_{T}(s)=\left[\frac{1}{\sqrt{[T s]}} \sum_{t=1}^{[T s]} g_{t T}\left(\hat{\beta}_{1}(s), \hat{\delta}(s)\right)\right]^{\prime} \hat{\Omega}_{1 T}^{-1}(s)\left[\frac{1}{\sqrt{[T s]}} \sum_{t=1}^{[T s]} g_{t T}\left(\hat{\beta}_{1}(s), \hat{\delta}(s)\right)\right]
$$

and

$$
O 2_{T}(s)=\left[\frac{1}{\sqrt{(T-[T s])}} \sum_{t=[T s]+1}^{[T]} g_{t T}\left(\hat{\beta}_{2}(s), \hat{\delta}(s)\right)\right]^{\prime} \hat{\Omega}_{2 T}^{-1}(s)\left[\frac{1}{\sqrt{(T-[T s])}} \sum_{t=[T s]+1} g_{t T}\left(\hat{\beta}_{2}(s), \hat{\delta}(s)\right)\right]
$$

Focusing first on size, all tests, except the modified tests, have rejection frequencies that are quite good, with some overrejection except for the $O$ test statistics which slightly underrejects. The supremum and exponential versions of the test statistics show some overrejection while the average version exhibits rejection frequencies closer to the desired sizes. The $L M$ test statistics (supremum, average and exponential mappings) significantly underreject for all DGPs (1 to 3) under the null and have poor power for other DGPs. For these reasons, the rejection frequencies for the $L M$ test statistics are not reported in the tables.

The modified tests (IPSCM, IPSCM $1^{I}, I P S C M 2^{I}$ and $I P S C M^{O}$ ) based on implied probabilities have rejection frequencies that are much too large. The intuition for this is that the modified test statistics contain a more volatile term in the denominator which can inflate the value of the tests and hence increase the rejection frequencies. ${ }^{5}$

The study of power is divided into two cases. In case 1, structural change occurs in the parameter $\theta$ while in case 2 , structural change occurs in the overidentifying restrictions. Under the alternative of instability in the parameter, $H_{A}^{I}$ (DGP 4 to 6 ), we see that the newly proposed test statistics based on implied probabilities have good rejection frequencies. The power of these test statistics is 
greater than the power of the standard Wald and $L R$ tests. The $I P S C^{O}$ and $O$ test statistics, geared toward instability in the overidentifying restrictions, have no useful power while the more general specification test, IPSC, has some reasonable power.

Under the alternative of instability in the overidentifying restrictions, e.g. $H_{A}^{O}$, (DGP 7 to 10$)$, we see that the test statistics specially designed to detect a change in the parameter $I P S C^{I}$, and the standard $W$ ald and $L R$ tests have little power while the targeted IPSC $O$ and $O$ tests have very good power. Importantly the tests based on implied probabilities are seen to have significantly higher power than $O$ tests for all cases. In some cases, the gain in power can be twice as important. As expected, the general specification tests based on implied probabilities, IPSC, have rejection frequencies that fall in between those of $I P S C^{O}$ and $I P S C^{I}$.

The increase in the autoregressive coefficient from 0 to 0.8 does not impact greatly on the rejection frequencies under the null hypothesis but under the alternative hypotheses the magnitude of the change is important. Under $H_{A}^{I}$, for example, we see that power is close to unity when the change in the autoregressive parameter is quite extreme (0 to 0.8$)$. Under $H_{A}^{O}$, which captures a change in the overidentifying restrictions, an increase (in absolute terms) in the moving average coefficient increases power.

Overall, structural change tests based on impled probabilities have very good size and power properties.

\section{Conclusion}

As noted by Back and Brown (1993), implied probabilities obtained from estimation of models using estimating equations could be used as an additional tool for model specification in the researcher's tool kit. An important specification test that has received considerable attention in the econometric literature has been a test for structural stability of either the underlying key parameters composing the estimation equations and, or, the stability of the additional equations (the overidentifying restrictions) that are often used in estimation. In this paper we have focused on the class of estimators based on the Generalized Empirical Likelihood approach. This approach is appealing intuitively because it requires the search for a vector of weights, one for each observation, that yields the most probable data distribution. We view these weights as potentially containing information on the content of the moment conditions (the estimating equations). In a pure entropy setting with no estimating equations the vector of weights is found to be maximally uninformative. That is, we obtain weights fluctuating around $1 / T$ where $T$ is the sample size. With constraints, the weights are chosen to be as maximally uninformative as the constraints will allow. 
In this sense, the weights make use of the available information contained in the sample. In particular we use the weights to detect an unknown structural change. We suggest different types of testing procedures for the detection of a structural change each based on different measures of the discrepancy between the estimated weights and the unconstrained weights $\frac{1}{T}$. Specifically, we propose general structural change tests to detect instability both in the identifying restrictions and in the overidentifying restrictions, instability in the identifying restrictions and instability in the overidentifying restrictions. We also propose a boundedly pivotal structural change test based on implied probabilities robust to cases of weak identification and cases in which parameters are completely unidentified. We found that tests based on these implied probabilities have good finite sample size and competitive power properties. An important attractive feature is that these tests are computed in a single step which eliminates the need to compute the weighting matrix required to perform for alternative structural change tests in GEL and for GMM estimation and testing. This weighting matrix is often linked to the poor finite sample properties of GMM-based estimators and test statistics. An issue that was not investigated in length in this paper was the impact of smoothing (to take into account serial dependence) on the performance of the tests. This interesting avenue of research is left for future work. 


\section{$7 \quad$ Appendix}

\subsection{Assumptions}

We consider triangular arrays because they are required to derived asymptotic results under the Pitman drift alternatives. Define $\mathbf{X}$ to be the domain of $g(\cdot, \theta)$ to include the support of $X_{T t}, \forall t$ and $\forall T$. Let $B_{0}$ and $\Delta_{0}$ denote compact subsets of $R^{r}$ and $R^{\nu}$ that contain neighborhoods of $\beta_{0}$ and $\delta_{0}$ in the parameter spaces $B$ and $\Delta$. Finally, let $\mu_{T t}$ denote the distribution of $X_{T t}$ and let $\bar{\mu}_{T}=(1 / T) \sum_{t=1}^{T} \mu_{T t}$. Throughout the Appendix, w.p.a.1 means with probability approaching one; $\|\cdot\|$ denotes the Euclidean norm of a vector or matrix; $\stackrel{p}{\rightarrow}$ and $\stackrel{d}{\rightarrow}$ denote respectively convergence in probability and in distribution and $\Rightarrow$ denotes weak convergence as defined by Pollard (1984, pp. 64-66).

Assumption 7.1. $\left\{x_{T t}: t \leq T, T \geq 1\right\}$ is a triangular array of $\mathbf{X}$-valued rv's that is $L^{0}$-near epoch dependent (NED) on a strong mixing base $\left\{Y_{T t}: t=\ldots, 0,1, \ldots ; T \geq 1\right\}$, where $\mathbf{X}$ is a Borel subset of $R^{k}$, and $\left\{\mu_{T t}: T \geq 1\right\}$ is tight on $\mathbf{X} .^{6}$

Define the smoothed moment conditions as: ${ }^{7}$

$$
g_{t T}(\beta, \delta)=\frac{1}{R_{T}} \sum_{r=t-T}^{t-1} k\left(\frac{r}{R_{T}}\right) g\left(x_{T t-r}, \beta, \delta\right)
$$

for an appropriate kernel. From now on, we consider the uniform kernel proposed by Kitamura and Stutzer (1997):

$$
g_{t T}(\beta, \delta)=\frac{1}{2 K_{T}+1} \sum_{r=-K_{T}}^{K_{T}} g\left(x_{T t-r}, \beta, \delta\right) .
$$

Assumption 7.2. $K_{T} / T^{2} \rightarrow 0$ and $K_{T} \rightarrow \infty$ as $T \rightarrow \infty$ and $K_{T}=O_{p}\left(T^{\frac{1}{2 \eta}}\right)$ for some $\eta>1^{8}$.

Assumption 7.3. For some $d>\max \left(2, \frac{2 \eta}{\eta-1}\right),\left\{g\left(x_{T t}, \beta, \delta\right): t \leq T, T \geq 1\right\}$ is a triangular array of mean zero $R^{q}$-valued random variables that are $L^{2}$-near epoch dependent of size $-\frac{1}{2}$ on a strong mixing base $\left\{Y_{T t}: t=\ldots, 0,1, \ldots ; T \geq 1\right\}$, of size $-d /(d-2)$ and $\sup \left\|g\left(x_{T t}, \beta \delta\right)\right\|^{d}<\infty$.

Assumption 7.4. $\operatorname{Var}\left(\frac{1}{\sqrt{T}} \sum_{t=1}^{T s} g\left(x_{T t}, \beta, \delta\right)\right) \rightarrow s \Omega \forall s \in[0,1]$ for some positive definite $q \times q$ matrix $\Omega$.

The above assumptions are sufficient to yield weak convergence of the standardized partial sum of the smoothed moment conditions under the null and the alternatives. In the following, $x_{t}$ is used to denote $x_{T t}$ for notational simplicity.

Assumption 7.5. $\tilde{g}\left(\beta_{0}, \delta_{0}\right)=0$ with $\left(\beta_{0}, \delta_{0}\right) \in B \times \Delta$ where $\tilde{g}\left(\beta_{0}, \delta_{0}\right)=\lim _{T \rightarrow \infty} \sum_{t=1}^{T} E g\left(x_{t}, \beta, \delta\right)$ and $B$ and $\Delta$ are bounded subsets of $R^{r}$ and $R^{\nu}, g\left(x_{t}, \beta, \delta\right)$ is continuous in $x$ for all $(\beta, \delta) \in B \times \Delta$ and is continuous in $(\beta, \delta)$ uniformly over $(\beta, \delta, x) \in B \times \Delta \times C$ for all compact sets $C \subset \mathbf{X}$.

Assumption 7.6. For every neighborhood $\Theta_{0} \subset \Theta$ of $\theta_{0}, \inf _{s \in S}\left(\inf _{\theta \in \Theta / \Theta_{0}}\|g(\theta, s)\|\right)>0$ where $g(\theta, s)=\left(s \tilde{g}\left(\beta_{1}, \delta\right)^{\prime},(1-s) \tilde{g}\left(\beta_{2}, \delta\right)^{\prime}\right)^{\prime}$.

Assumption 7.7. (a) $\rho(\cdot)$ is twice continuously differential and concave on its domain, an open interval $\Phi$ containing $0, \rho_{1}=\rho_{2}=-1 ;(\boldsymbol{b}) \gamma(s) \in \Gamma(s)$ where $\Gamma(s)=\left\{\gamma(s):\|\gamma(s)\| \leq D\left(T /\left(K_{T}^{2}\right)^{-\zeta}\right\}\right.$ for some $D>0$ with $\frac{1}{2}>\zeta>\frac{1}{d(1-1 / \eta)}$. 
Assumption 7.7 (b) is similar to the assumption in Newey and Smith (2004) and Smith (2004) but for $\gamma(s)=\left(\gamma_{1}(s)^{\prime}, \gamma_{2}(s)^{\prime}\right)^{\prime}$. It specifies bounds on $\gamma(s)$ and with the existence of higher than second moments in Assumption 7.3, it leads to the arguments $\gamma(s)^{\prime} g_{t T}(\theta, s)$ being in the domain $\Phi$ of $\rho(\cdot)$ w.p.a. 1 in the first subsample for all $\beta_{1}, \delta$ and $1 \leq t \leq[T s]$ and in the second subsample for all $\beta_{2}, \delta$ and $[T s]+1 \leq t \leq T$.

Under Assumptions 7.1, 7.2, 7.3, 7.5, 7.6 and 7.7, Guay and Lamarche (2009) show for the partial-sample GEL that $\sup _{s \in S}\left\|\hat{\theta}_{T}(s)-\theta_{0}\right\| \stackrel{p}{\rightarrow} 0, \sup _{s \in S}\left\|\hat{\gamma}_{T}(s)\right\| \stackrel{p}{\rightarrow} 0,\left\|\hat{\gamma}_{T}(s)\right\|=$ $O_{p}\left(T / K_{T}^{2}\right)^{-1 / 2}$ and $\sup _{s \in S}\left\|\frac{1}{T} \sum_{t=1}^{T} g_{t T}\left(\hat{\theta}_{T}(s), s\right)\right\|=O_{p}\left(T^{-1 / 2}\right)$.

The consistency of the full-sample GEL estimator is obtained by slight modifications of Assumptions 7.6 and 7.7 (b). Assumption 7.6 must be modified by a simplified version with $\tilde{g}(\beta, \delta)$ instead of $g(\theta, s)$. Assumption 7.7 (b) holds but for the full sample Lagrange multiplier $\gamma$. The consistency result that $\tilde{\theta}_{T} \stackrel{p}{\rightarrow} \theta_{0}$ is then derived under weaker conditions than in Smith (2004).

The following high level assumptions are sufficient to derive the weak convergence under the null and the alternative of the PS-GEL estimators $\hat{\theta}_{T}(s)$ and $\hat{\gamma}_{T}(s)$ (see Guay and Lamarche, 2009). These assumptions are similar to the ones in Andrews (1993).

Assumption 7.8. $\sup _{s \in S}\left\|\hat{\Omega}_{i T}(s)-\Omega\right\| \stackrel{p}{\rightarrow} 0$ where $\Omega$ is defined in Section 3.1 and $S$ whose closure lies in $(0,1)$ for $i=1,2$.

Assumption 7.8 holds under conditions given in Andrews (1991) and Lemma A.3 in Smith (2004). To respect these conditions, Assumption 7.3 can be replaced by the following assumption:

Assumption $\mathbf{7 . 3}^{\prime} \cdot\left\{g\left(x_{T t}, \theta\right): t \leq T, T \geq 1\right\}$ is a triangular array of mean zero $R^{q}$-valued rv's that is $\alpha$-mixing with mixing coefficients $\sum_{j=1}^{\infty} j^{2} \alpha(j)^{(\nu-1) / \nu}<\infty$ for some $\nu>1$ and $\sup _{t \leq T, T \geq 1} E\left\|g\left(x_{T t}, \theta\right)\right\|^{d}<\infty$ for some $d>\max \left(4 \nu, \frac{2 \eta}{\eta-1}\right)$.

Assumptions $7.3^{\prime}$ and 7.8 guarantee for the full-sample and partial-sample GEL that

$$
\tilde{\Omega}_{T}=\frac{2 K+1}{T} \sum_{t=1}^{T} g_{t T}\left(\tilde{\beta}_{T}, \tilde{\delta}_{T}\right) g_{t T}\left(\tilde{\beta}_{T}, \tilde{\delta}_{T}\right)^{\prime} \stackrel{p}{\rightarrow} \Omega
$$

and

$$
\hat{\Omega}_{T}(s)=\frac{2 K+1}{T} \sum_{t=1}^{T} g_{t T}\left(\hat{\theta}_{T}(s), s\right) g_{t T}\left(\hat{\theta}_{T}(s), s\right)^{\prime} \stackrel{p}{\rightarrow} \Omega(s) .
$$

Now, let $G(\beta, \delta)=\lim _{T \rightarrow \infty} \frac{1}{T} \sum_{t=1^{T}} E\left[\partial g\left(x_{t}, \beta, \delta\right) / \partial\left(\beta^{\prime}, \delta^{\prime}\right)\right]$ and $G=G\left(\beta_{0}, \delta_{0}\right)$.

Assumption 7.9. $g(x, \beta, \delta)$ is differentiable in $(\beta, \delta), \forall(\beta, \delta) \in B_{0} \times \Delta_{0} \forall x \in \mathbf{X}_{\mathbf{0}} \subset \mathbf{X}$ for a Borel measurable set $\mathbf{X}_{\mathbf{0}}$ that satisfies $P\left(x_{t} \in \mathbf{X}_{\mathbf{0}}\right)=1 \forall t \leq T, T \geq 1, g(x, \beta, \delta)$ is 
Borel measurable in $x \forall(\beta, \delta) \in B_{0} \times \Delta_{0}, \partial g\left(x_{t}, \beta, \delta\right) / \partial\left(\beta^{\prime}, \delta^{\prime}\right)$ is continuous in $(x, \beta, \delta)$ on $\mathbf{X} \times B_{0} \times \Delta_{0}$,

$$
\sup _{1 \leq t \leq T} E\left[\sup _{(\beta, \delta) \in B_{0} \times \Delta_{0}}\left\|\partial g\left(x_{t}, \beta, \delta\right) / \partial\left(\beta^{\prime}, \delta^{\prime}\right)\right\|^{d /(d-1)}\right]<\infty
$$

and $\operatorname{rank}(G)=p+\nu$.

Assumption 7.10. $\lim _{T \rightarrow \infty} \frac{1}{T} \sum_{t=1}^{T s} E g_{t T}(\beta, \delta)$ exists uniformly over $(\beta, \delta, s) \in B \times \Delta \times S$ and equals $s \lim _{T \rightarrow \infty} \frac{1}{T} \sum_{t=1}^{T} E g\left(x_{t}, \beta, \delta\right)=s \tilde{g}(\beta, \delta)$.

Assumption 7.11. $\lim _{T \rightarrow \infty} \frac{1}{T} \sum_{t=1}^{T s} E \partial g_{t T}\left(\beta_{0}, \delta_{0}\right) / \partial\left(\beta^{\prime}, \delta^{\prime}\right)$ exists uniformly over $s \in S$ and equals $s G \forall s \in S$ and $S$ whose closure lies in $(0,1)$.

Assumption 7.12. $G(s)^{\prime} \Omega(s)^{-1} G(s)$ is nonsingular $\forall s \in S$ and has eigenvalues bounded away from zero $\forall s \in S$ and $S$ whose closure lies in $(0,1)$.

\subsection{Lemmas}

The following Lemmas are necessary to establish the proofs of the Theorems:

Lemma 7.1. We denote $\{B(s): s \in[0,1]\}$ as q-dimensional vectors of mutually independent Brownian motion on $[0,1]$ and define

$$
J(s)=\left[\begin{array}{c}
\Omega^{1 / 2} B(s) \\
\Omega^{1 / 2}(B(1)-B(s))
\end{array}\right]
$$

where $B(\pi)$ is a q-dimensional vector of standard Brownian motion. Under Assumptions 7.1 to 7.12, every sequence of PS-GEL estimators $\{\hat{\theta}(\cdot), \hat{\gamma}(\cdot), T \geq 1\}$ satisfies

$$
\begin{aligned}
\sqrt{T}\left(\hat{\theta}_{T}(\cdot)-\theta_{0}\right) & \Rightarrow-\left(G(\cdot)^{\prime} \Omega(\cdot)^{-1} G(\cdot)\right)^{-1} G(\cdot)^{\prime} \Omega(\cdot)^{-1} J(\cdot), \\
\frac{\sqrt{T}}{2 K_{T}+1} \hat{\gamma}_{T}(\cdot) & \Rightarrow-\left(\Omega(\cdot)^{-1}-\Omega(\cdot)^{-1}\left(G(\cdot)^{\prime} \Omega(\cdot)^{-1} G(\cdot)\right)^{-1} G(\cdot)^{\prime} \Omega(\cdot)^{-1}\right) J(\cdot)
\end{aligned}
$$

as a process indexed by $s \in S$, where $S$ has closure in $(0,1)$. Further, the sequence of GEL estimators $\hat{\theta}_{T}(\cdot)$ and the sequence of auxiliary estimators $\hat{\gamma}_{T}(\cdot)$ are asymptotically uncorrelated. Under the alternative (8), the same results hold except that:

$$
J(s)=\left[\begin{array}{c}
\Omega^{1 / 2} B(s)+H(s) \\
\Omega^{1 / 2}(B(1)-B(s))+H(1)-H(s)
\end{array}\right]
$$

where $H(s)=\int_{0}^{s} h(r) d r$ with $h(r)=h(\eta, \tau, r)$ to simplify the notation.

Proof of Lemma 7.1: see Guay and Lamarche (2009). 
Lemma 7.2. Under Assumptions 7.1 to 7.12, for the unrestricted implied probabilities evaluated at the restricted estimator, we get

$$
\pi_{t}^{G E L}\left(\tilde{\theta}_{T}, s\right)=\frac{1}{T}+\frac{1}{T} g_{t T}\left(\tilde{\theta}_{T}, s\right)^{\prime} \widetilde{\gamma}_{T}(s)\left(1+o_{p}(1)\right)+O_{p}\left(K_{T} / T^{2}\right)
$$

and $\pi_{t}^{G E L}\left(\tilde{\theta}_{T}\right)=\frac{1}{T}+o_{p}(1)$ uniformly in $t=1, \ldots, T$. According to the notation in Definition 3.4, for $t=1, \ldots,[T s]$,

$$
T \pi_{t}^{G E L}\left(\tilde{\theta}_{T}, s\right)-1=g_{t T}\left(\tilde{\beta}_{T}, \tilde{\delta}_{T}\right)^{\prime} \widetilde{\gamma}_{1 T}\left(1+o_{p}(1)\right)+O_{p}\left(K_{T} / T\right)
$$

and for $t=[T s]+1, \ldots, T$,

$$
T \pi_{t}^{G E L}\left(\tilde{\theta}_{T}, s\right)-1=g_{t T}\left(\tilde{\beta}_{T}, \tilde{\delta}_{T}\right)^{\prime} \widetilde{\gamma}_{2 T}\left(1+o_{p}(1)\right)+O_{p}\left(K_{T} / T\right) .
$$

\section{Proof of Lemma 7.2}

We need to derive the asymptotic distribution of the partial-sample implied probabilities evaluated at the full-sample estimator:

$$
\pi_{t}^{G E L}\left(\tilde{\theta}_{T}, s\right)=\frac{\rho_{1}\left(\widetilde{\gamma}_{T}(s)^{\prime} g_{t T}\left(\widetilde{\theta}_{T}, s\right)\right)}{\sum_{t=1}^{T} \rho_{1}\left(\widetilde{\gamma}_{T}(s)^{\prime} g_{t T}\left(\widetilde{\theta}_{T}, s\right)\right)} .
$$

A mean value expansion for $\pi_{t}^{G E L}\left(\tilde{\theta}_{T}, s\right)$ around $\left(\tilde{\theta}_{T}, \tilde{\gamma}_{T}\right)=\left(\tilde{\theta}_{T}, 0\right)$ yields:

$$
\begin{aligned}
\pi_{t}^{G E L}\left(\tilde{\theta}_{T}, s\right)= & \frac{1}{T}+\frac{\rho_{2}\left(\bar{\gamma}_{T}(s)^{\prime} g_{t T}\left(\widetilde{\theta}_{T}, s\right)\right)}{\sum_{t=1}^{T} \rho_{1}\left(\widetilde{\gamma}_{T}(s)^{\prime} g_{t T}\left(\widetilde{\theta}_{T}, s\right)\right)} g_{t T}\left(\widetilde{\theta}_{T}, s\right)^{\prime} \widetilde{\gamma}_{T}(s)- \\
& \frac{\rho_{1}\left(\widetilde{\gamma}_{T}(s)^{\prime} g_{t T}\left(\widetilde{\theta}_{T}, s\right)\right)}{\left[\sum_{t=1}^{T} \rho_{1}\left(\bar{\gamma}_{T}(s)^{\prime} g_{t T}\left(\widetilde{\theta}_{T}, s\right)\right)\right]^{2}} \sum_{t=1}^{T} \rho_{2}\left(\bar{\gamma}_{T}(s)^{\prime} g_{t T}\left(\widetilde{\theta}_{T}, s\right)\right) g_{t T}\left(\widetilde{\theta}_{T}, s\right)^{\prime} \widetilde{\gamma}_{T}(s)
\end{aligned}
$$

where $\bar{\gamma}_{T}(s)$ lies on the line segment joining $\widetilde{\gamma}_{T}(s)$ and 0 and may differ from row to row.

Since $\tilde{\gamma}_{T}(s)$ converges in probability to 0 and using Lemma 4.3 in Guay and Lamarche (2009), this yields that $\rho_{j}\left(\bar{\gamma}_{T}(s)^{\prime} g_{t T}\left(\widetilde{\theta}_{T}, s\right)\right)=\rho_{j}(0)+o_{p}(1)=-1+o_{p}(1)$, for $j=1,2, \forall t$. Thus, we get:

$\pi_{t}^{G E L}\left(\tilde{\theta}_{T}, s\right)=\frac{1}{T}+\frac{1}{T}\left[g_{t T}\left(\tilde{\theta}_{T}, s\right)^{\prime} \widetilde{\gamma}_{T}(s)\left(1+o_{p}(1)\right)\right]-\frac{1}{T^{2}}\left[\sum_{t=1}^{T} g_{t T}\left(\tilde{\theta}_{T}, s\right)^{\prime} \widetilde{\gamma}_{T}(s)\left(1+o_{p}(1)\right)\right]$.

As $\sum_{t=1}^{T} g\left(\tilde{\theta}_{T}\right)=\sum_{t=1}^{T} g_{t T}\left(\tilde{\theta}_{T}\right)+O_{p}\left(K^{2} /(2 K+1)\right)=O_{p}\left(T^{1 / 2}\right)$ which implies $\sum_{t=1}^{T} g_{t T}\left(\tilde{\theta}_{T}, s\right)=$ $O_{p}\left(T^{1 / 2}\right), g_{t T}\left(\tilde{\theta}_{T}, s\right)=O_{p}\left(\left(2 K_{T}+1\right)^{-1 / 2}\right)$ and $\tilde{\gamma}_{T}(s)=O_{p}\left(2 K_{T}+1 / \sqrt{T}\right){ }^{9}$ yields:

$$
\pi_{t}^{G E L}\left(\tilde{\theta}_{T}, s\right)=\frac{1}{T}+\frac{1}{T}\left[g_{t T}\left(\tilde{\theta}_{T}, s\right)^{\prime} \tilde{\gamma}_{T}(s)\left(1+o_{p}(1)\right)\right]+O_{p}\left(K_{T} / T^{2}\right)
$$

and

$$
\pi_{t}^{G E L}\left(\tilde{\theta}_{T}, s\right)=\frac{1}{T}\left(1+o_{p}(1)\right)
$$


uniformly in $t=1, \ldots, T$. Thus, we get:

$$
T \pi_{t}^{G E L}\left(\tilde{\theta}_{T}, s\right)-1=g_{t T}\left(\tilde{\beta}_{T}, \tilde{\delta}_{T}\right)^{\prime} \tilde{\gamma}_{1 T}\left(1+o_{p}(1)\right)+O_{p}\left(K_{T} / T\right)
$$

uniformly in $t=1, \ldots,[T s]$ and

$$
T \pi_{t}^{G E L}\left(\tilde{\theta}_{T}, s\right)-1=g_{t T}\left(\tilde{\beta}_{T}, \tilde{\delta}_{T}\right)^{\prime} \tilde{\gamma}_{2 T}\left(1+o_{p}(1)\right)+O_{p}\left(K_{T} / T\right)
$$

uniformly in $t=[T s]+1, \ldots, T$.

\subsection{Proofs of Theorems}

\section{Proof of Theorem 4.1}

We expand the FOC of the Lagrange multiplier vector for the partial-sample GEL evaluated at the restricted estimator in a Taylor series about 0 for the first part of the sample $t=$ $1, \ldots,[T s]$. Thus

$$
\begin{aligned}
\frac{1}{T} \sum_{t=1}^{[T s]} \rho_{1}\left(\tilde{\gamma}_{1 T}^{\prime} g_{t T}\left(\tilde{\beta}_{T}, \tilde{\delta}_{T}\right)\right) g_{t T}\left(\tilde{\beta}_{T}, \tilde{\delta}_{T}\right) & =-\frac{1}{T} \sum_{t=1}^{[T s]} g_{t T}\left(\tilde{\beta}_{T}, \tilde{\delta}_{T}\right)-\frac{1}{T} \sum_{t=1}^{[T s]} g_{t T}\left(\tilde{\beta}_{T}, \tilde{\delta}_{T}\right) g_{t T}\left(\tilde{\beta}_{T}, \tilde{\delta}_{T}\right)^{\prime} \tilde{\gamma}_{1 T} \\
& +\frac{1}{T} \sum_{t=1}^{[T s]} g_{t T}\left(\tilde{\beta}_{T}, \tilde{\delta}_{T}\right) \sum_{j=2}^{\infty} \frac{1}{j !} \rho_{j+1}(0)\left(g_{t T}\left(\tilde{\beta}_{T}, \tilde{\delta}_{T}\right)^{\prime} \tilde{\gamma}_{1 T}\right)^{j}
\end{aligned}
$$

where $\tilde{\gamma}_{1 T}$ is the partial-sample Lagrange multiplier evaluated for the restricted estimator for $t=1, \ldots,[T s]$ and $\rho_{1}(0)=\rho_{2}(0)=-1$. By the fact that $g_{t T}\left(\tilde{\beta}_{T}, \tilde{\delta}_{T}\right)=O_{p}\left(\left(2 K_{T}+1\right)^{-1 / 2}\right)$, this yields

$$
\begin{aligned}
\frac{1}{T} \sum_{t=1}^{[T s]} \rho_{1}\left(\tilde{\gamma}_{1 T}^{\prime} g_{t T}\left(\tilde{\beta}_{T}, \tilde{\delta}_{T}\right)\right) g_{t T}\left(\tilde{\beta}_{T}, \tilde{\delta}_{T}\right) & =-\frac{1}{T} \sum_{t=1}^{[T s]} g_{t T}\left(\tilde{\beta}_{T}, \tilde{\delta}_{T}\right)-\frac{1}{T} \sum_{t=1}^{[T s]} g_{t T}\left(\tilde{\beta}_{T}, \tilde{\delta}_{T}\right) g_{t T}\left(\tilde{\beta}_{T}, \tilde{\delta}_{T}\right)^{\prime} \tilde{\gamma}_{1 T} \\
& +O_{p}\left(\left(2 K_{T}+1\right)^{-3 / 2}\left\|\tilde{\gamma}_{1 T}\right\|^{2}\right) .
\end{aligned}
$$

By using a consistent estimator $\tilde{\Omega}_{T}=\frac{2 K+1}{[T s]} \sum_{t=1}^{[T s]} g_{t T}\left(\tilde{\beta}_{T}, \tilde{\delta}_{T}\right) g_{t T}\left(\tilde{\beta}_{T}, \tilde{\delta}_{T}\right)^{\prime}$ and $\tilde{\gamma}_{1 T}=O_{p}\left(\left(2 K_{T}+1\right) / \sqrt{T}\right)$, we get under the null:

$$
0=\frac{1}{T} \sum_{t=1}^{[T s]} g_{t T}\left(\tilde{\beta}_{T}, \tilde{\delta}_{T}\right)+\frac{s}{2 K+1} \tilde{\Omega}_{T} \tilde{\gamma}_{1 T}+O_{p}\left(\left(2 K_{T}+1\right)^{-3 / 2}\left\|\tilde{\gamma}_{1 T}\right\|^{2}\right)
$$

which yields

$$
\frac{s}{2 K_{T}+1} \tilde{\gamma}_{1 T}=-\tilde{\Omega}_{T}^{-1} \frac{1}{T} \sum_{t=1}^{[T s]} g_{t T}\left(\tilde{\beta}_{T}, \tilde{\delta}_{T}\right)+O_{p}\left(K_{T}^{1 / 2} / T\right) .
$$


Now, expanding this expression around $\beta_{0}$ and $\delta_{0}$ gives:

$$
\frac{s \sqrt{T}}{2 K_{T}+1} \tilde{\gamma}_{1 T}=-\tilde{\Omega}_{T}^{-1} \frac{1}{\sqrt{T}} \sum_{t=1}^{[T s]} g_{t T}\left(\beta_{0}, \delta_{0}\right)-s \tilde{\Omega}_{T}^{-1} G \sqrt{T}\left(\begin{array}{c}
\tilde{\beta}_{T}-\beta_{0} \\
\tilde{\delta}_{T}-\delta_{0}
\end{array}\right)+o_{p}(1) .
$$

We can easily show for the restricted estimators under the null that (see also Smith, 2004):

$$
\sqrt{T}\left(\begin{array}{c}
\tilde{\beta}_{T}-\beta_{0} \\
\tilde{\delta}_{T}-\delta_{0}
\end{array}\right)=-\left(G^{\prime} \Omega^{-1} G\right)^{-1} G^{\prime} \Omega^{-1} \frac{1}{\sqrt{T}} \sum_{t=1}^{T} g_{t T}\left(\beta_{0}, \delta_{0}\right)+o_{p}(1) .
$$

Combining the two preceding results, we obtain

$\frac{s \sqrt{T}}{2 K_{T}+1} \tilde{\gamma}_{1 T}=-\Omega^{-1} \frac{1}{\sqrt{T}} \sum_{t=1}^{[T s]} g_{t T}\left(\beta_{0}, \delta_{0}\right)+s \Omega^{-1} G\left(G^{\prime} \Omega^{-1} G\right)^{-1} G^{\prime} \Omega^{-1} \frac{1}{\sqrt{T}} \sum_{t=1}^{T} g_{t T}\left(\beta_{0}, \delta_{0}\right)+o_{p}(1)$.

By Lemma 5.1 in Guay and Lamarche (2009), under the null of no structural change, we have

$$
\Omega^{-1 / 2} \frac{1}{\sqrt{T}} \sum_{t=1}^{[T s]} g_{t T}\left(\beta_{0}, \delta_{0}\right) \Rightarrow B(s)
$$

and under the generic alternative (8),

$$
\Omega^{-1 / 2} \frac{1}{\sqrt{T}} \sum_{t=1}^{[T s]} g_{t T}\left(\beta_{0}, \delta_{0}\right) \Rightarrow B(s)+\Omega^{-1 / 2} H(s)
$$

where $B(s)$ is a $q$-vector of standard Brownian motions and $H(s)=\int_{0}^{s} h(r) d r$. Note also that:

$$
\frac{1}{T} \sum_{t=1}^{[T s]} g_{t T}\left(\beta_{0}, \delta_{0}\right)=\frac{1}{T} \sum_{t=1}^{[T s]} g_{t}\left(\beta_{0}, \delta_{0}\right)+o_{p}(1)
$$

Using Lemma 7.2 and the derivation above,

$$
\begin{aligned}
T \pi_{t}^{G E L}\left(\tilde{\theta}_{T}, s\right)-1= & -\left(\frac{2 K+1}{s} \tilde{\Omega}_{T}^{-1} \frac{1}{T} \sum_{t=1}^{[T s]} g_{t T}\left(\tilde{\beta}_{T}, \tilde{\delta}_{T}\right)+O_{p}\left(K_{T}^{3 / 2} / T\right)\right)^{\prime} \times \\
& g_{t T}\left(\tilde{\beta}_{T}, \tilde{\delta}_{T}\right)\left(1+o_{p}(1)\right)+O_{p}\left(K_{T} / T\right) .
\end{aligned}
$$

Let us now examine the asymptotic distribution of the expression on the right-hand side. First, we show the following asymptotic result for the partial sums of the moments conditions evaluated at $\tilde{\theta}_{T}$ :

$$
\Omega^{-1 / 2}\left[\frac{1}{\sqrt{T}} \sum_{t=1}^{[T s]} g_{t T}\left(\tilde{\beta}_{T}, \tilde{\delta}_{T}\right)\right] \Rightarrow B(s)-s \Omega^{-1 / 2} G\left[G^{\prime} \Omega^{-1} G\right]^{-1} G^{\prime} \Omega^{-1 / 2} B(1) .
$$

By a mean value expansion:

$$
\frac{1}{\sqrt{T}} \sum_{t=1}^{[T s]} g_{t T}\left(\tilde{\beta}_{T}, \tilde{\delta}_{T}\right)=\frac{1}{\sqrt{T}} \sum_{t=1}^{[T s]} g_{t T}\left(\beta_{0}, \delta_{0}\right)+\frac{1}{T} \sum_{t=1}^{[T s]} \frac{\partial g_{t T}\left(\bar{\beta}_{T}, \bar{\delta}_{T}\right)}{\partial\left(\beta^{\prime}, \delta^{\prime}\right)}\left(\begin{array}{c}
\tilde{\beta}_{T}-\beta_{0} \\
\tilde{\delta}_{T}-\delta_{0}
\end{array}\right)
$$


where $\bar{\beta}_{T}$ lies on the line segment joining $\tilde{\beta}_{T}$ and $\beta_{0}$ and may differ from row to row and respectively for $\bar{\delta}_{T}$. By applying eq. (20), we obtain

$$
\frac{1}{\sqrt{T}} \sum_{t=1}^{[T s]} g_{t T}\left(\tilde{\beta}_{T}, \tilde{\delta}_{T}\right)=\frac{1}{\sqrt{T}} \sum_{t=1}^{[T s]} g_{t T}\left(\beta_{0}, \delta_{0}\right)-s G\left[G^{\prime} \Omega^{-1} G\right]^{-1} G^{\prime} \Omega^{-1} \frac{1}{T} \sum_{t=1}^{T} g_{t T}\left(\beta_{0}, \delta_{0}\right)+o_{p}(1) .
$$

It follows by (21) that

$$
\Omega^{-1 / 2} \frac{1}{\sqrt{T}} \sum_{t=1}^{[T s]} g_{t T}\left(\tilde{\beta}_{T}, \tilde{\delta}_{T}\right) \Rightarrow B(s)-s \Omega^{-1 / 2} G\left[G^{\prime} \Omega^{-1} G\right]^{-1} G^{\prime} \Omega^{-1 / 2} B(1) .
$$

By (23), the following partial sum can be shown to be:

$$
\begin{aligned}
\sum_{t=1}^{[T s]}\left[T \pi_{t}^{G E L}\left(\tilde{\theta}_{T}, s\right)-1\right]^{2}= & \left(\frac{2 K+1}{s} \tilde{\Omega}_{T}^{-1} \frac{1}{T} \sum_{t=1}^{[T s]} g_{t T}\left(\tilde{\beta}_{T}, \tilde{\delta}_{T}\right)\right)^{\prime} \sum_{t=1}^{[T s]} g_{t T}\left(\tilde{\beta}_{T}, \tilde{\delta}_{T}\right) g_{t T}\left(\tilde{\beta}_{T}, \tilde{\delta}_{T}\right)^{\prime} \\
& \times\left(\frac{2 K+1}{s} \tilde{\Omega}_{T}^{-1} \frac{1}{T} \sum_{t=1}^{[T s]} g_{t T}\left(\tilde{\beta}_{T}, \tilde{\delta}_{T}\right)\right)+o_{p}(1) .
\end{aligned}
$$

Considering that $\tilde{\Omega}_{T}=\frac{2 K+1}{[T s]} \sum_{t=1}^{[T s]} g_{t T}\left(\tilde{\beta}_{T}, \tilde{\delta}_{T}\right) g_{t T}\left(\tilde{\beta}_{T}, \tilde{\delta}_{T}\right)^{\prime}$, the expression above gives:

$$
\sum_{t=1}^{[T s]}\left[T \pi_{t}^{G E L}\left(\tilde{\theta}_{T}, s\right)-1\right]^{2}=\frac{2 K+1}{s}\left(\frac{1}{\sqrt{T}} \sum_{t=1}^{[T s]} g_{t T}\left(\tilde{\beta}_{T}, \tilde{\delta}_{T}\right)\right)^{\prime} \tilde{\Omega}_{T}^{-1}\left(\frac{1}{\sqrt{T}} \sum_{t=1}^{[T s]} g_{t T}\left(\tilde{\beta}_{T}, \tilde{\delta}_{T}\right)\right)+o_{p}(1) .
$$

By the result above, the partial sum of interest yields by equations (26) and the consistency of $\tilde{\Omega}_{T}$ :

$$
\begin{aligned}
\frac{s}{2 K+1} \sum_{t=1}^{[T s]}\left[T \pi_{t}^{G E L}\left(\tilde{\theta}_{T}, s\right)-1\right]^{2} \Rightarrow & {\left[B(s)-s \Omega^{-1 / 2} G\left[G^{\prime} \Omega^{-1} G\right]^{-1} G^{\prime} \Omega^{-1 / 2} B(1)\right]^{\prime} \times } \\
& {\left[B(s)-s \Omega^{-1 / 2} G\left[G^{\prime} \Omega^{-1} G\right]^{-1} G^{\prime} \Omega^{-1 / 2} B(1)\right] . }
\end{aligned}
$$

The expression $\left[B(s)-s \Omega^{-1 / 2} G\left[G^{\prime} \Omega^{-1} G\right]^{-1} G^{\prime} \Omega^{-1 / 2} B(1)\right]$ can be rewritten as:

$$
\left[I-P_{G}\right] B(s)+P_{G}[B(s)-s B(1)]
$$

with $P_{G}=\Omega^{-1 / 2} G\left[G^{\prime} \Omega^{-1} G\right]^{-1} G^{\prime} \Omega^{-1 / 2}$. We can now decompose:

$$
\Omega^{-1 / 2} G\left(G^{\prime} \Omega^{-1} G\right)^{-1} G^{\prime} \Omega^{-1 / 2}=C^{\prime} \Lambda C
$$

where $C C^{\prime}=I$ and

$$
\Lambda=\left[\begin{array}{ll}
I_{p} & 0 \\
0 & 0
\end{array}\right]
$$

and $I_{p}$ is an identity matrix with dimension $p \times p$ where $p=r+\nu$ with $r$ and $\nu$ the respective dimension of the vectors $\beta$ and $\delta$. Note also that $C B(s)$ has the same asymptotic distribution as $B(s)$. The RHS of expression (28) can then be rewritten as:

$$
B(s)^{\prime}\left[I-C^{\prime} \Lambda C\right] B(s)+[B(s)-s B(1)]^{\prime} C^{\prime} \Lambda C[B(s)-s B(1)]
$$


which is equal in distribution to

$$
B_{(q-p)}(s)^{\prime} B_{(q-p)}(s)+B B_{p}(s)^{\prime} B B_{p}(s)
$$

where $B_{(q-p)}(s)$ is a $(q-p)$-vector of standard Brownian motion and $B B_{p}(s)=B_{p}(s)-s B_{p}(1)$ is a $p$-vector of Brownian bridge. The result follows. The asymptotic distribution under the alternative (8) can be easily obtained similarly using (22).

\section{Proof of Theorem 4.3}

By Lemma 7.2 applied to the difference between the partial-sample implied probabilities evaluated at the unrestricted and at the restricted estimators we get:

$$
T\left[\hat{\pi}_{t}\left(\hat{\theta}_{T}(s), s\right)-\tilde{\pi}_{t}\left(\tilde{\theta}_{T}, s\right)\right]=g_{t T}\left(\hat{\theta}_{T}(s), s\right)^{\prime} \hat{\gamma}_{T}(s)-g_{t T}\left(\tilde{\theta}_{T}, s\right)^{\prime} \tilde{\gamma}_{T}(s)+o_{p}\left(T^{-1 / 2}\right) .
$$

Let us define the following selection matrices:

$$
H_{1}=\left[\begin{array}{lll}
I_{r \times r} & 0_{r \times r} & 0_{r \times \nu} \\
0_{\nu \times r} & 0_{\nu \times r} & I_{\nu \times \nu}
\end{array}\right]
$$

and

$$
H_{2}=\left[\begin{array}{lll}
0_{r \times r} & I_{r \times r} & 0_{r \times \nu} \\
0_{\nu \times r} & 0_{\nu \times r} & I_{\nu \times \nu}
\end{array}\right]
$$

and the corresponding estimator: $\hat{\theta}_{1 T}(s)=H_{1} \hat{\theta}_{T}(s)=\left(\hat{\beta}_{1 T}(s)^{\prime}, \hat{\delta}_{T}(s)^{\prime}\right)^{\prime}$ and $\hat{\theta}_{2 T}(s)=$ $H_{2} \hat{\theta}_{T}(s)=\left(\hat{\beta}_{2 T}(s)^{\prime}, \hat{\delta}_{T}(s)^{\prime}\right)^{\prime}$. Similarly, for the restricted estimator $\tilde{\theta}_{1 T}(s)=H_{1} \tilde{\theta}_{T}(s)=$ $\left(\tilde{\beta}_{T}(s)^{\prime}, \tilde{\delta}_{T}(s)^{\prime}\right)^{\prime}$ and $\tilde{\theta}_{2 T}(s)=H_{2} \tilde{\theta}_{T}(s)=\left(\tilde{\beta}_{T}(s)^{\prime}, \hat{\delta}_{T}(s)^{\prime}\right)^{\prime}$ where $\tilde{\theta}_{T}(s)=\left(\tilde{\beta}_{T}(s)^{\prime}, \tilde{\beta}_{T}(s)^{\prime}, \tilde{\delta}_{T}(s)^{\prime}\right)^{\prime}$. Accordingly, we define $\theta_{1,0}=H_{1} \theta_{0}=\left(\beta_{0}^{\prime}, \delta_{0}^{\prime}\right)^{\prime}$ and $\theta_{2,0}=H_{2} \theta_{0}=\left(\beta_{0}^{\prime}, \delta_{0}^{\prime}\right)^{\prime}$.

Let us examine the expression for the first part of the sample, namely $t=1, \ldots,[T s]$. Replacing the unrestricted and the restricted estimators of $\gamma$ by the corresponding expression (19), we get:

$$
\begin{aligned}
T\left[\pi_{t}\left(\hat{\theta}_{T}(s), s\right)-\pi_{t}\left(\tilde{\theta}_{T}, s\right)\right]= & -\left[\left(2 K_{T}+1\right) \Omega(s)^{-1} \frac{1}{[T s]} \sum_{t=1}^{[T s]} g_{t T}\left(\hat{\theta}_{T}(s), s\right)+o_{p}(1)\right]^{\prime} g_{t T}\left(\hat{\theta}_{T}(s), s\right)\left(1+o_{p}(1)\right) \\
& +\left[\left(2 K_{T}+1\right) \Omega(s)^{-1} \frac{1}{[T s]} \sum_{t=1}^{[T s]} g_{t T}\left(\tilde{\theta}_{T}, s\right)+o_{p}(1)\right]^{\prime} g_{t T}\left(\tilde{\theta}_{T}, s\right)\left(1+o_{p}(1)\right) \\
& +O_{p}\left(K_{T} / T\right)
\end{aligned}
$$

which equals

$$
\begin{aligned}
T\left[\pi_{t}\left(\hat{\theta}_{T}(s), s\right)-\pi_{t}\left(\tilde{\theta}_{T}, s\right)\right]= & -\left[\frac{1}{[T s]} \sum_{t=1}^{[T s]} g_{t T}\left(\hat{\theta}_{1 T}(s)\right)-\frac{1}{[T s]} \sum_{t=1}^{[T s]} g_{t T}\left(\tilde{\theta}_{1 T}\right)+o_{p}(1)\right]^{\prime}(30) \\
& \times \Omega^{-1}\left(2 K_{T}+1\right) g_{t T}\left(\hat{\theta}_{1 T}(s)\right)\left(1+o_{p}(1)\right)+O_{p}\left(K_{T} / T\right)
\end{aligned}
$$


since $G_{t T}(\cdot)=O_{p}\left(\left(2 K_{T}+1\right)^{-1 / 2}\right)$ and $\hat{\theta}_{1 T}(s)-\tilde{\theta}_{1 T}=O_{p}\left(T^{-1 / 2}\right)$.

Now, consider the following mean value expansion:

$$
\frac{1}{[T s]} \sum_{t=1}^{[T s]} g_{t T}\left(\tilde{\theta}_{1 T}\right)=\frac{1}{[T s]} \sum_{t=1}^{[T s]} g_{t T}\left(\hat{\theta}_{1 T}(s)\right)+\frac{1}{[T s]} \sum_{t=1}^{[T s]} \frac{\partial g_{t T}\left(\bar{\theta}_{1 T}(s)\right)}{\partial \theta_{1}^{\prime}}\left(\tilde{\theta}_{1 T}-\hat{\theta}_{1 T}(s)\right) .
$$

where $\bar{\theta}_{1 T}(s)$ lies on the line segment joining $\hat{\theta}_{1 T}(s)$ and $\tilde{\theta}_{1 T}$ and may differ from row to row. Thus,

$$
\begin{aligned}
T\left[\pi_{t}\left(\hat{\theta}_{T}(s), s\right)-\pi_{t}\left(\tilde{\theta}_{T}, s\right)\right]= & {\left[\frac{1}{[T s]} \sum_{t=1}^{[T s]} \frac{\partial g_{t T}\left(\bar{\theta}_{1 T}(s)\right)}{\partial \theta_{1}^{\prime}}\left(\tilde{\theta}_{1 T}-\hat{\theta}_{1 T}(s)\right)+o_{p}(1)\right]^{\prime} } \\
& \times \Omega^{-1}\left(2 K_{T}+1\right) g_{t T}\left(\hat{\theta}_{1 T}(s)\right)\left(1+o_{p}(1)\right)+O_{p}\left(K_{T} / T\right) .
\end{aligned}
$$

The partial sum over the first subsample for the square of the LHS expression above yields:

$\frac{1}{2 K+1} \sum_{t=1}^{[T s]}\left[T \pi_{t}\left(\hat{\theta}_{T}(s), s\right)-T \pi_{t}\left(\tilde{\theta}_{T}, s\right)\right]^{2}=[T s]\left(\tilde{\theta}_{1 T}-\hat{\theta}_{1 T}(s)\right)^{\prime} G^{\prime} \Omega^{-1} G\left(\tilde{\theta}_{1 T}-\hat{\theta}_{1 T}(s)\right)+o_{p}(1)$

as $\left(2 K_{T}+1\right) \frac{1}{[T s]} \sum_{t=1}^{[T s]} g_{t T}\left(\hat{\theta}_{1 T}(s), s\right) g_{t T}\left(\hat{\theta}_{1 T}(s), s\right)^{\prime}$ is a consistent estimator of $\Omega$.

Similarly for the second subsample $t=[T s]+1, \ldots, T$, we obtain

$\frac{1}{2 K+1} \sum_{t=[T s]+1}^{T}\left[T \pi_{t}\left(\hat{\theta}_{T}(s), s\right)-T \pi_{t}\left(\tilde{\theta}_{T}, s\right)\right]^{2}=(T-[T s])\left(\tilde{\theta}_{2 T}-\hat{\theta}_{2 T}(s)\right)^{\prime} G^{\prime} \Omega^{-1} G\left(\tilde{\theta}_{2 T}-\hat{\theta}_{2 T}(s)\right)+o_{p}(1)$

By results derived above and some calculations, this yields

$\frac{1}{2 K+1} \sum_{t=1}^{T}\left[T \pi_{t}\left(\hat{\theta}_{T}(s), s\right)-T \pi_{t}\left(\tilde{\theta}_{T}, s\right)\right]^{2}=T\left(\tilde{\theta}_{T}-\hat{\theta}_{T}(s)\right)^{\prime} G\left(\theta_{0}, s\right)^{\prime} \Omega^{-1}(s) G\left(\theta_{0}, s\right)\left(\tilde{\theta}_{T}-\hat{\theta}_{T}(s)\right)+o_{p}(1)$.

Andrews (1993, p. 851-852) shows that this expression is asymptotically equivalent to the $L M_{T}(s)$ statistic for parameters instability. This gives the result under the null. Under the alternative, Guay and Lamarche (2009) show that the $L M_{T}(s)$ statistic for the restricted GEL estimator has the same asymptotic distribution as in Theorem 4.3. Since the asymptotic equivalence between the expression above and the $L M_{T}(s)$ statistic, the result follows.

\section{Proof of Theorem 4.4}

The two subsamples are now evaluated separately. A direct application of Lemma 7.2 but for the estimation of the subsample with the first $[T s]$ observations gives

$$
[T s] \pi_{t}^{G E L}\left(\hat{\theta}_{1 T}(s), s\right)-1=g_{t T}\left(\hat{\theta}_{1 T}(s)\right)^{\prime} \hat{\gamma}_{1 T}(s)+o_{p}\left([T s]^{-1 / 2}\right)
$$


uniformly in $t=1, \ldots,[T s]$. This is obtained with a proof similar to the one of Lemma 7.2 but only for the first part of the sample. This yields

$$
\left([T s] \pi_{t}^{G E L}\left(\hat{\theta}_{1 T}(s), s\right)-1\right)^{2}=\hat{\gamma}_{1 T}(s)^{\prime} g_{t T}\left(\hat{\theta}_{1 T}(s)\right) g_{t T}\left(\hat{\theta}_{1 T}(s)^{\prime} \hat{\gamma}_{1 T}(s)\right)+o_{p}\left([T s]^{-1}\right) .
$$

By summing the expression above to $t=\ldots,[T s]$, and by (19) for the unrestricted estimator applied to the sample $t=1, \ldots,[T s]$, this yields

$$
\begin{aligned}
\sum_{t=1}^{[T s]}\left([T s] \pi_{t}^{G E L}\left(\hat{\theta}_{1 T}(s), s\right)-1\right)^{2}= & \left(2 K_{T}+1\right)^{2} \frac{1}{\sqrt{[T s]}} \sum_{t=1}^{[T s]} g_{t T}\left(\hat{\theta}_{1 T}(s)\right)^{\prime} \Omega^{-1} \frac{1}{[T s]} \sum_{t=1}^{[T s]} g_{t T}\left(\hat{\theta}_{1 T}(s)\right) \\
& \times\left(g_{t T}\left(\hat{\theta}_{1 T}(s)\right)\right)^{\prime} \Omega^{-1} \frac{1}{\sqrt{[T s]}} \sum_{t=1}^{[T s]} g_{t T}\left(\hat{\theta}_{1 T}(s)\right)+o_{p}(1) .
\end{aligned}
$$

As $\left(2 K_{T}+1\right) \frac{1}{[T s]} \sum_{t=1}^{[T s]} g_{t T}\left(\hat{\theta}_{1 T}(s)\right) g_{t T}\left(\hat{\theta}_{1 T}(s)\right)^{\prime}$ is a consistent estimator of $\Omega$, this gives

$$
\frac{1}{2 K_{T}+1} \sum_{t=1}^{[T s]}\left([T s] \pi_{t}^{G E L}\left(\hat{\theta}_{1 T}(s), s\right)-1\right)^{2}=\frac{1}{\sqrt{[T s]}} \sum_{t=1}^{[T s]} g_{t T}\left(\hat{\theta}_{1 T}(s)\right)^{\prime} \Omega^{-1} \frac{1}{\sqrt{[T s]}} \sum_{t=1}^{[T s]} g_{t T}\left(\hat{\theta}_{1 T}(s)\right)+o_{p}(1) .(33)
$$

Similarly, we can easily obtain that

$$
\begin{gathered}
\frac{1}{2 K_{T}+1} \sum_{t=[T s]+1}^{T}\left((T-[T s]) \pi_{t}^{G E L}\left(\hat{\theta}_{2 T}(s), s\right)-1\right)^{2}= \\
\frac{1}{\sqrt{T-[T s]}} \sum_{t=[T s]+1}^{T} g_{t T}\left(\hat{\theta}_{2 T}(s)\right)^{\prime} \Omega^{-1} \frac{1}{\sqrt{T-[T s]}} \sum_{t=[T s]+1}^{T} g_{t T}\left(\hat{\theta}_{2 T}(s)\right)+o_{p}(1) .
\end{gathered}
$$

Now, by a mean value expansion and the consistency of $\hat{\theta}_{T}(s)$,

$\Omega(s)^{-1 / 2} \frac{1}{\sqrt{T}} \sum_{t=1}^{T} g_{t T}\left(\hat{\theta}_{T}(s), s\right)=\Omega(s)^{-1 / 2}\left[\begin{array}{c}\frac{1}{\sqrt{T}} \sum_{t=1}^{[T s]} g_{t T}\left(\beta_{0}, \delta_{0}\right) \\ \frac{1}{\sqrt{T}} \sum_{t=[T s]+1}^{T} g_{t T}\left(\beta_{0}, \delta_{0}\right)\end{array}\right]+\Omega(s)^{-1 / 2} G(s) \sqrt{T}\left(\hat{\theta}_{T}(s)-\theta_{0}\right)+o_{p}(1)$.

By Lemma 7.1 and (21),

$$
\begin{aligned}
\Omega(s)^{-1 / 2} \frac{1}{\sqrt{T}} \sum_{t=1}^{T} g_{t T}\left(\hat{\theta}_{T}(s), s\right) \Rightarrow & {\left[\begin{array}{c}
\frac{1}{\sqrt{s}} B(s) \\
\frac{1}{\sqrt{(1-s)}}(B(1)-B(s))
\end{array}\right]-\Omega(s)^{-1 / 2} G(s)\left(G(s)^{\prime} \Omega(s)^{-1} G(s)\right)^{-1} } \\
& \times G(s)^{\prime} \Omega(s)^{-1 / 2}\left[\begin{array}{c}
B(s) \\
(B(1)-B(s))
\end{array}\right] .
\end{aligned}
$$

The RHS can be rewritten as

$$
\left(I-\Omega(s)^{-1 / 2} G(s)\left(G(s)^{\prime} \Omega(s)^{-1} G(s)\right)^{-1} G(s)^{\prime} \Omega(s)^{-1 / 2}\right)\left[\begin{array}{c}
\frac{1}{\sqrt{s}} B(s) \\
\frac{1}{\sqrt{(1-s)}}(B(1)-B(s))
\end{array}\right]
$$


Since the entire parameter vector is estimated for both subsample, e.g. $\hat{\theta}_{1 T}(s)=\hat{\beta}_{1 T}(s)$ and $\hat{\theta}_{2 T}(s)=\hat{\beta}_{2 T}(s)$, the matrices $\Omega(s)$ and $G(s)$ are block-diagonal and by the definition of $g_{t T}\left(\hat{\theta}_{T}(s), s\right)$, we get:

$$
\Omega^{-1 / 2} \frac{1}{\sqrt{T}} \sum_{t=1}^{[T s]} g_{t T}\left(\hat{\beta}_{1 T}(s)\right) \Rightarrow\left(I_{q}-\Omega^{-1 / 2} G_{\beta}\left(G_{\beta}^{\prime} \Omega^{-1} G_{\beta}\right)^{-1} G_{\beta}^{\prime} \Omega^{-1 / 2}\right) B(s)
$$

and

$\Omega^{-1 / 2} \frac{1}{\sqrt{T}} \sum_{t=[T s]+1}^{T} g_{t T}\left(\hat{\beta}_{2 T}(s)\right) \Rightarrow\left(I_{q}-\Omega^{-1 / 2} G_{\beta}\left(G_{\beta}^{\prime} \Omega^{-1} G_{\beta}\right)^{-1} G_{\beta}^{\prime} \Omega^{-1 / 2}\right)(B(1)-B(s))$.

We can now decompose:

$$
\left(I-\Omega^{-1 / 2} G_{\beta}\left(G_{\beta}^{\prime} \Omega^{-1} G_{\beta}\right)^{-1} G^{\prime} \Omega^{-1 / 2}\right)=\left(I-C^{\prime} \Lambda C\right)
$$

where $C C^{\prime}=I$ and

$$
\Lambda=\left[\begin{array}{ll}
I_{r} & 0 \\
0 & 0
\end{array}\right]
$$

and $I_{r}$ is an identity matrix with dimension $r \times r+$ with $r$ is the dimension of the vectors $\beta$. Noting that $C\left(I-C^{\prime} \Lambda C\right) B(s)=(I-\Lambda) C B(s)$ and $C B(s)$ is also a $r$-vector of Brownian motion. Now consider the multiplication of the RHS term of equation (35) by the matrix $C$, this yields

$$
C \Omega^{-1 / 2} \frac{1}{\sqrt{T}} \sum_{t=1}^{[T s]} g_{t T}\left(\hat{\beta}_{1 T}(s)\right) \Rightarrow\left(I_{q}-\Lambda\right) B(s) .
$$

Using this result, we obtain directly the asymptotic distribution of the statistic for the first subsample by equation (33) considering that $C^{\prime} C=I_{q}$. The proof is similar for the second subsample (eq. 34), hence obtaining the asymptotic distribution under the null.

Under the alternative (8), the asymptotic distribution under the alternative is derived similarly using Lemma 7.1.

\section{Proof of Theorem 4.5}

Define

$$
P(\theta(s), \gamma(s), s)=\sum_{t=1}^{T} \frac{\left[\rho\left(k \gamma(s)^{\prime} g_{t T}(\theta, s)\right)-\rho_{0}\right]}{T}
$$

and $\hat{g}_{T}(\theta, s)=\frac{1}{T} \sum_{t=1}^{T} g_{t T}(\theta, s)$.

Since $\left.\tilde{\theta}_{T}(s)=\arg \min _{\theta \in \Theta} P(\theta, \gamma(s)), s\right)$

$$
\sup _{\gamma(s) \in \tilde{\Gamma}_{T}(s)} P\left(\tilde{\theta}_{T}(s), \gamma(s), s\right) \leq \sup _{\gamma(s) \in \tilde{\Gamma}_{T}(s)} P\left(\theta_{0}, \gamma(s), s\right)
$$


and this holds for all $s \in S$. Let $\bar{\gamma}_{T}(s)=\arg \max _{\gamma_{s} \in \Gamma_{T}(s)} P\left(\theta_{0}, \gamma(s), s\right)$ and $\dot{\gamma}(s)=\tau \bar{\gamma}(s), 0 \leq$ $\tau \leq 1$ and we have $\left.\left(2 K_{T}+1\right) \sum_{t=1}^{T} \rho_{2}\left(\dot{\gamma}(s)^{\prime} g_{t T}\left(\theta_{0}, s\right)\right) g_{t T}\left(\theta_{0}, s\right) g_{t T} \theta_{0}, s\right)^{\prime} / T \stackrel{p}{\rightarrow}-\Omega(s)$. By a second-order Taylor expansion with Lagrange remainder,

$$
\begin{aligned}
\frac{1}{2 K_{T}+1} P\left(\theta_{0}, \bar{\gamma}(s), s\right) & =-\left(\frac{\bar{\gamma}(s)}{2 K_{T}+1}\right)^{\prime} \hat{g}_{T}\left(\theta_{0}, s\right) \\
& +\left(\frac{\bar{\gamma}(s)}{2 K_{T}+1}\right)^{\prime}\left(\sum_{t=1}^{T} \rho_{2}\left(\dot{\gamma}(s)^{\prime} g_{t T}\left(\theta_{0}, s\right) \hat{g}_{t T}\left(\theta_{0}, s\right) g_{t T}\left(\theta_{0}, s\right)^{\prime} / T\right) \bar{\gamma}(s) / 2\right. \\
& =\hat{g}_{T}\left(\theta_{0}, s\right)^{\prime} \Omega(s)^{-1} \hat{g}_{T}\left(\left(\theta_{0}, s\right)-\hat{g}_{T}\left(\theta_{0}, s\right)^{\prime} \Omega(s)^{-1} \hat{g}_{T}\left(\theta_{0}, s\right) / 2+o_{p}(1)\right. \\
& =\hat{g}_{T}\left(\theta_{0}, s\right)^{\prime} \Omega(s)^{-1} \hat{g}_{T}\left(\theta_{0}, s\right) / 2+o_{p}(1)
\end{aligned}
$$

w.p.a.1 where the second equality holds by $\frac{1}{2 K_{T}+1} \bar{\gamma}(s)=-\tilde{\Omega}(s)^{-1} \hat{g}_{T}\left(\theta_{0}, s\right)+o_{p}(1)$. Similarly, we can obtain

$$
\frac{1}{2 K_{T}+1} P\left(\tilde{\theta}_{T}(s), \tilde{\gamma}(s), s\right)=\hat{g}_{T}\left(\tilde{\theta}_{T}(s), s\right)^{\prime} \tilde{\Omega}_{T}(s)^{-1} \hat{g}_{T}\left(\tilde{\theta}_{T}(s), s\right) / 2+o_{p}(1)
$$

with $\tilde{\gamma}_{T}(s)=\arg \max _{\gamma_{s} \in \Gamma_{T}(s)} P\left(\tilde{\theta}_{T}(s), \gamma(s), s\right)$ and

$$
\tilde{\Omega}_{T}(s)=\left[\begin{array}{cc}
s \tilde{\Omega}_{1 T}(s) & 0 \\
0 & (1-s) \tilde{\Omega}_{2 T}(s)
\end{array}\right]
$$

with

$$
\tilde{\Omega}_{1 T}(s)=\frac{2 K_{T}+1}{[T s]} \sum_{t=1}^{[T s]} g_{t T}\left(\tilde{\beta}_{T}(s)\right) g_{t T}\left(\tilde{\beta}_{T}(s)\right)^{\prime}
$$

and

$$
\tilde{\Omega}_{2 T}(s)=\frac{2 K_{T}+1}{T-[T s]} \sum_{t=[T s]+1}^{T} g_{t T}\left(\tilde{\beta}_{T}(s)\right) g_{t T}\left(\tilde{\beta}_{T}(s)\right)^{\prime}
$$

Next, we show that the statistic $\operatorname{IPSC}_{T}^{R, G E L}(s)$ is asymptotically equivalent at first-order to $\frac{2}{2 K_{T}+1} P\left(\tilde{\theta}_{T}(s), \tilde{\gamma}(s), s\right)$. The following partial sum evaluated at the restricted partial-sample GEL estimator yields:

$$
\begin{aligned}
\sum_{t=1}^{[T s]}\left[T \pi_{t}^{G E L}\left(\tilde{\theta}_{T}(s), s\right)-1\right]^{2}= & \left(\frac{2 K+1}{s} \tilde{\Omega}_{1 T}(s)^{-1} \frac{1}{T} \sum_{t=1}^{[T s]} g_{t T}\left(\tilde{\beta}_{T}\right)\right)^{\prime} \sum_{t=1}^{[T s]} g_{t T}\left(\tilde{\beta}_{T}(s)\right) g_{t T}\left(\tilde{\beta}_{T}(s)\right)^{\prime} \\
& \times\left(\frac{2 K+1}{s} \tilde{\Omega}_{1 T}(s)^{-1} \frac{1}{T} \sum_{t=1}^{[T s]} g_{t T}\left(\tilde{\beta}_{T}(s)\right)\right)+o_{p}(1) .
\end{aligned}
$$

Considering that $\tilde{\Omega}_{1 T}(s)=\frac{2 K+1}{[T s]} \sum_{t=1}^{[T s]} g_{t T}\left(\tilde{\beta}_{T}(s)\right) g_{t T}\left(\tilde{\beta}_{T}(s)\right)^{\prime}$, the expression above gives:

$$
\sum_{t=1}^{[T s]}\left[T \pi_{t}^{G E L}\left(\tilde{\theta}_{T}(s), s\right)-1\right]^{2}=\frac{2 K+1}{s}\left(\frac{1}{\sqrt{T}} \sum_{t=1}^{[T s]} g_{t T}\left(\tilde{\beta}_{T}(s)\right)\right)^{\prime} \tilde{\Omega}_{1 T}(s)^{-1}\left(\frac{1}{\sqrt{T}} \sum_{t=1}^{[T s]} g_{t T}\left(\tilde{\beta}_{T}(s)\right)\right)+o_{p}(1) .
$$


Similarly for the second subsample

$$
\sum_{t=[T s]+1}^{T}\left[T \pi_{t}^{G E L}\left(\tilde{\theta}_{T}(s), s\right)-1\right]^{2}=\frac{2 K+1}{1-s}\left(\frac{1}{\sqrt{T}} \sum_{t=[T s]+1}^{T} g_{t T}\left(\tilde{\beta}_{T}(s)\right)\right)^{\prime} \tilde{\Omega}_{2 T}(s)^{-1}\left(\frac{1}{\sqrt{T}} \sum_{t=[T s]+1}^{T} g_{t T}\left(\tilde{\beta}_{T}(s)\right)\right)+o_{p}(1) .
$$

Then, we get for the statistic

$$
\begin{aligned}
\operatorname{IPSC}_{T}^{R, G E L}(s) & =\left(\frac{1}{\sqrt{T}} \sum_{t=1}^{[T s]} g_{t T}\left(\tilde{\beta}_{T}(s)\right)\right)^{\prime} \frac{\tilde{\Omega}_{1 T}(s)^{-1}}{s}\left(\frac{1}{\sqrt{T}} \sum_{t=1}^{[T s]} g_{t T}\left(\tilde{\beta}_{T}(s)\right)\right) \\
& +\left(\frac{1}{\sqrt{T}} \sum_{t=[T s]+1}^{T} g_{t T}\left(\tilde{\beta}_{T}(s)\right)\right)^{\prime} \frac{\tilde{\Omega}_{2 T}(s)^{-1}}{1-s}\left(\frac{1}{\sqrt{T}} \sum_{t=[T s]+1}^{T} g_{t T}\left(\tilde{\beta}_{T}(s)\right)\right)+o_{p}(1) \\
& =T \hat{g}_{T}\left(\tilde{\theta}_{T}(s), s\right)^{\prime} \tilde{\Omega}_{T}(s)^{-1} \hat{g}_{T}\left(\tilde{\theta}_{T}(s), s\right)+o_{p}(1)
\end{aligned}
$$

with $\tilde{\theta}_{T}(s)=\left(\tilde{\beta}_{T}(s)^{\prime}, \tilde{\beta}_{T}(s)^{\prime}\right)^{\prime}$. Since $\frac{2 T}{2 K_{T}+1} P\left(\tilde{\theta}_{T}(s), \tilde{\gamma}(s), s\right)$ is asymptotically bounded by $\frac{2 T}{2 K_{T}+1} P\left(\theta_{0}, \bar{\gamma}(s), s\right)$ for all $s \in S$ and that $I P S C_{T}^{R, G E L}(s)$ is asymptotically equivalent at firstorder to $\frac{2 T}{2 K_{T}+1} P\left(\tilde{\theta}_{T}(s), \tilde{\gamma}(s), s\right)$ for all $s \in S$, the statistic $\operatorname{IPSC} C_{T}^{R, G E L}(s)$ is asymptotically bounded for all $s \in S$ by the asymptotic distribution of $T \hat{g}_{T}\left(\theta_{0}, s\right)^{\prime} \Omega(s)^{-1} \hat{g}_{T}\left(\theta_{0}, s\right)$ which is derived in Caner (2007). The result under the null follows and the asymptotic bound under the alternative can be easily derived. 


\section{Footnotes}

${ }^{1}$ See in particular the special issue of Journal of Business and Economic Statistics, 1996, volume 14.

${ }^{2}$ The function $h(\cdot)$ allows for a wide range of alternative hypotheses (see Sowell (1996b)). In its generic form it can be expressed as the uniform limit of step functions, $\eta \in R^{i}, \tau \in R^{j}$ such that $0<\tau_{1}<\tau_{2}<\ldots<\tau_{j}<1$ and $\theta^{*}$ is in the interior of $\Theta$. Therefore it can accommodate multiple breaks.

${ }^{3}$ This specification allows for the overidentifying restrictions to be violated just after the breakpoint $\left(\eta_{1}=0\right.$ and $\left.\eta_{2} \neq 0\right)$, just before the breakpoint $\left(\eta_{1} \neq 0\right.$ and $\left.\eta_{2}=0\right)$ or both $\left(\eta_{1} \neq 0, \eta_{2} \neq 0\right.$ and $\left.\eta_{1} \neq \eta 2\right)$.

${ }^{4}$ This is verified by comparing the critical values in Hall and Sen (1999) and Guay (2003). The critical values in Table 1 in Hall and Sen for $q-r$ in our notation are the same than the critical values in Guay (2003) but for $2 q-2 r$.

${ }^{5}$ Only the results for the test IPSCM are included in the tables. The results for the other modified tests can be obtained upon request.

${ }^{6}$ For a definition of $L^{p}$-near epoch dependence and tightness, see Andrews (1993, p. 829-830). For a presentation of the concept of near epoch dependence, we refer the reader to Gallant and White (1988).

${ }^{7}$ Note here that $g_{t T}$ denotes the smoothed moment conditions and $x_{T t}$ a triangular array of random variables.

${ }^{8}$ This assumption is slightly different than that in Smith (2004) but facilitates the proofs at no real cost.

${ }^{9}$ See Kitamura and Stutzer (1997) and Guay and Lamarche (2009). 


\section{References}

Anatolyev, S. (2005) 'GMM, GEL, serial correlation, and asymptotic bias.' Econometrica $73(3), 983-1002$

Andrews, D.W.K. (1991) 'Heteroskedasticity and autocorrelation consistent covariance matrix estimation.' Econometrica 59, 817-858

_ (1993) 'Tests for parameter instability and structural change with unknown change point.' Econometrica $61,821-856$

Andrews, D.W.K., and J.C. Monahan (1992) 'An improved heteroskedasticity and autocorrelation consistent covariance matrix estimator.' Econometrica 60, 953-966

Andrews, D.W.K., and W. Ploberger (1994) 'Optimal tests when a nuisance parameter is present only under the alternative.' Econometrica 62 , 1383-1414

Antoine, B., H. Bonnal, and E. Renault (2007) 'On the efficient use of the informational content of estimating equations: implied probabilities and euclidean empirical likelihood.' Journal of Econometrics 138, 461-487

Back, K., and D.P. Brown (1993) 'Implied probabilities in GMM estimators.' Econometrica 61, 971-975

Caner, M. (2007) 'Bounded pivotal structural change tests in continuous updating GMM with strong, weak identification and completely unidentified cases.' Journal of Econometrics $137,28-67$

_ (2008a) 'Exponential tilting and weak instruments: estimation and testing.' Working Paper, North Carolina State University

_ (2008b) 'Nearly-singular design in GMM and generalized empirical likelihood estimators.' Journal of Econometrics 144, 511-523

Cressie, N., and T. Read (1984) 'Multinomial goodness-of-fit tests.' Journal of the Royal Statistical Society B 46, 440-464

Gallant, A.R. (1987) Nonlinear statistical models (New York: Wiley)

Gallant, A.R., and H. White (1988) A Unified theory of estimation and inference for nonlinear dynamic models (Basil Blackwell)

Ghysels, E., A. Guay, and A. Hall (1997) 'Predictive tests for structural change with unknown breakpoint.' Journal of Econometrics 82, 209-233 
Ghysels, E., and A. Hall (1990) 'Are consumption-based intertemporal capital asset pricing models structural.' Journal of Econometrics 45, 121-139

Gregory, A.W., Jean-Francois Lamarche, and Gregor W. Smith (2002) 'Information-theoretic estimation of preference parameters: macroeconomic applications and simulation evidence.' Journal of Econometrics 107, 213-233

Guay, A., and F. Pelgrin (2007) 'Using implied probabilities to improve estimation in unconditional moment models: Re-assessing the new Keynesian Phillips curve.' Manuscript

Guay, A., and J-F Lamarche (2009) 'Structural change tests for GEL criteria.' Working Paper

Guggenberger, P., and R. J. Smith (2008) 'Generalized empirical likelihood tests in time series models with potential identification failure.' Journal of Econometrics 142, 134-161

Guggenberger, P., J.J.S. Ramalho, and R. J. Smith (2007) 'GEL Pearson-type statistics under weak identification.' Manuscript

Hall, A.R., and A. Sen (1999) 'Structural stability testing in models estimated by generalized methods of moments.' Journal of Business and Economic Statistics 17, 335-348

Hall, P., and J.L. Horowitz (1996) 'Bootstrap critical values for tests based on generalizedmethod-of-moments estimators.' Econometrica 64, 891-916

Hansen, L.P. (1982) 'Large sample properties of generalized method of moments estimators.' Econometrica 50, 1029-1054

Hansen, L.P., J. Heaton, and A. Yaron (1996) 'Finite-sample properties of some alternative GMM estimators.' Journal of Business and Economic Statistics 14, 262-280

Imbens, G.W., R.H. Spady, and P. Johnson (1998) 'Information theoretic approaches to inference in moment condition models.' Econometrica 66, 333-357

Kitamura, Y. (2001) 'Asymptotic optimality of empirical likelihood for testing moment restrictions.' Econometrica 69, 1661-1672

Kitamura, Y., and M. Stutzer (1997) 'An information-theoretic alternative to generalized method of moments estimation.' Econometrica 65, 861-874

Kullback, S., and R.A. Leibler (1951) 'On information and sufficiency.' Annals of Mathematical Statistics 22, 79-86

Newey, W.K., and K.D. West (1987) 'A simple positive semidefinite, heteroskedastic and autocorrelation consistent covariance matrix.' Econometrica 55, 703-708 
_ (1994) 'Automatic lag selection in covariance matrix estimation.' Review of Economic Studies 61, 631-654

Newey, W.K., and R.J. Smith (2004) 'Higher order properites of GMM and generalized empirical likelihood estimators.' Econometrica 72, 219-256

Otsu, T. (2006) 'Generalized empirical likelihood inference for nonlinear and time series models under weak identification.' Econometric Theory 22, 513-527

Pollard, D. (1984) Convergence of Stochastic Processes (New York: Springer-Verlag)

Qin, J., and J. Lawless (1994) 'Empirical likelihood and general estimating equations.' Annals of Statistics 22, 300-325

Ramalho, J.J.S., and R. J. Smith (2005) 'Goodness of fit tests for moment condition models.' Working Paper 20005/05

Schennach, S.M. (2007) 'Point estimation with exponetially tilted empirical likelihood.' Annals of Statistics 35, 634-672

Shannon, C.E. (1948) 'Axiomatic derivation of the principle of maximum entropy and the principle of minimum cross-entropy.' IEEE Transactions on information theory $26,26-$ 37

Smith, R. J. (2004) 'GEL criteria for moment condition models.' CEMMAP Working Paper CWP19/04

Smith, R.J. (1997) 'Alternative semi-parametric likelihood approaches to generalized method of moments.' The Economic Journal 107, 503-519

Smith, R.J. (2000) 'Empirical likelihood estimation and inference.' In Applications of Differential Geometry to Econometrics, ed. M. Salmon and P. Marriott (Cambridge: Cambridge University Press) pp. 119-150

Sowell, F. (1996a) 'Optimal tests of parameter variation in the generalized method of moments framework.' Econometrica 64, 1085-1108

_ (1996b) 'Test for violations of moment conditions.' Manuscript, Carnegie Mellon University

Stock, James H., and J.H. Wright (2000) 'GMM with weak identification.' Econometrica $68,1055-1096$ 
Figure 1: Simulated Implied Probabilities
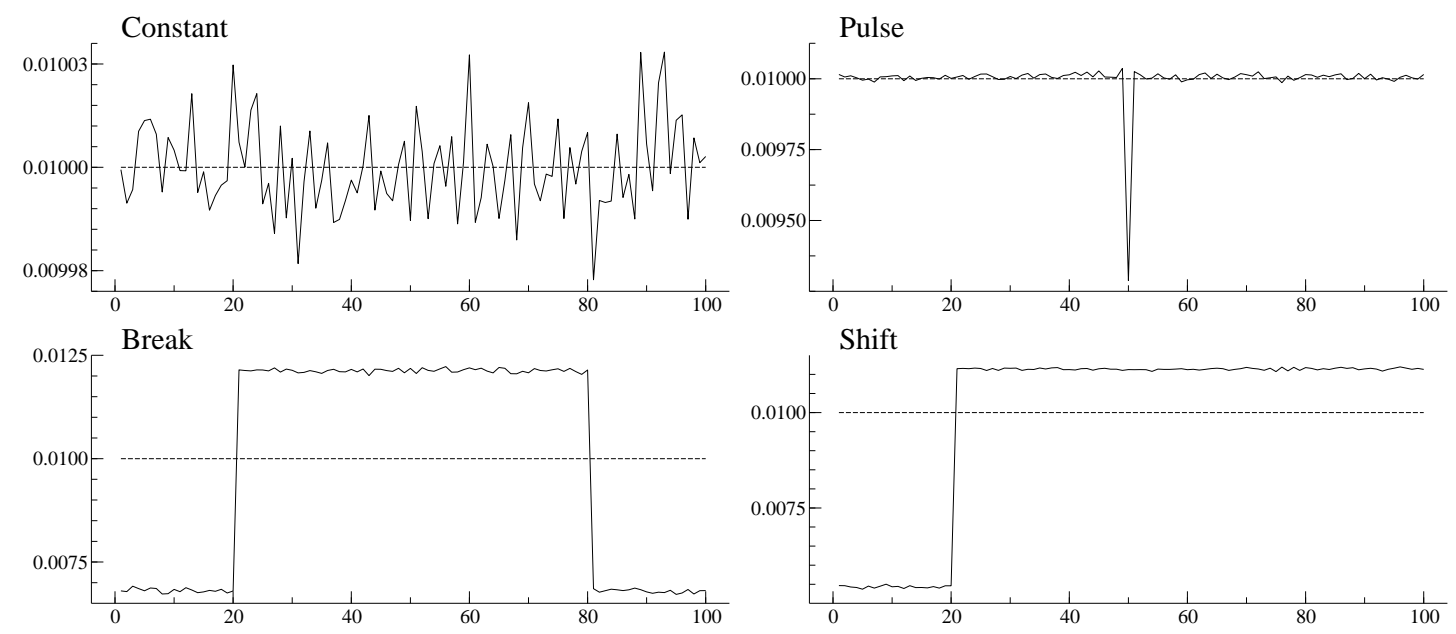

CRRA Parameter
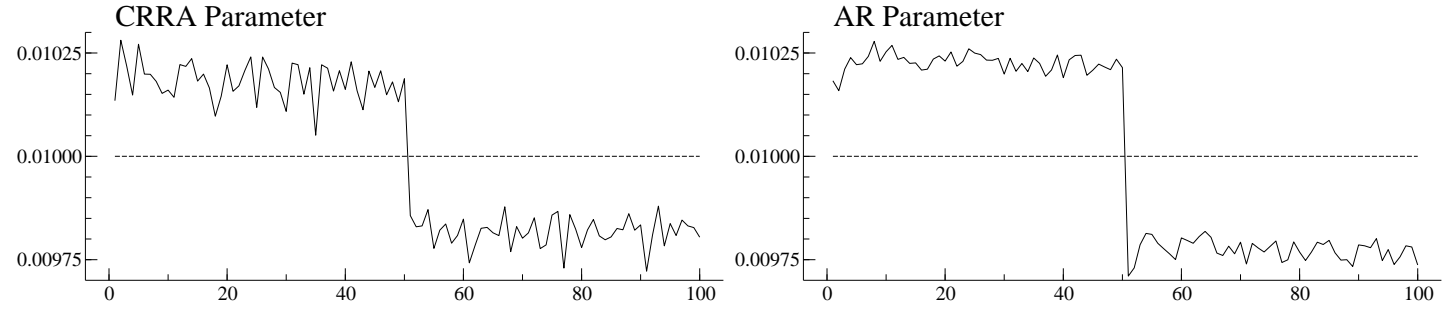

Notes: The constant case refers to no break, the pulse case to a one-time temporary jump at observation 50 , the break case to a change in the mean for 60 time periods and the shift to a permanent change in the mean at observation 20. For the CRRA case, a preference parameter is estimated using two moments. The moment conditions are violated at observation 51. For the AR case a autoregressive parameter is estimated. The data generating process is represented by an $A R(1)$ process for $t \leq 50$ and by an $A R M A(1,2)$ otherwise. The sample is of size 100 and in all cases the horizontal lines correspond to the empirical weights of 1/100. 


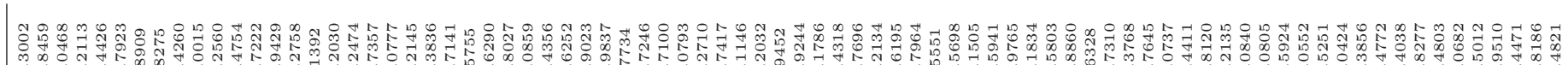
近囬向

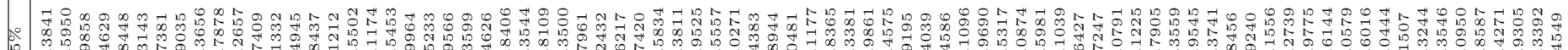
jós

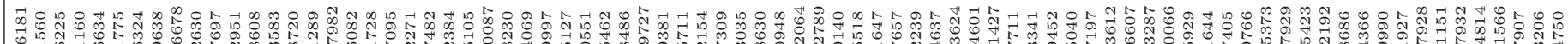

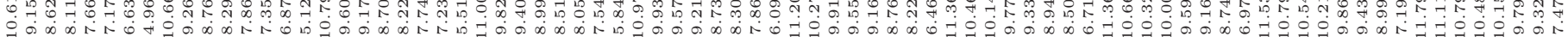

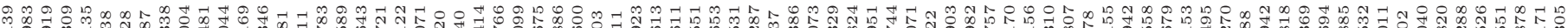

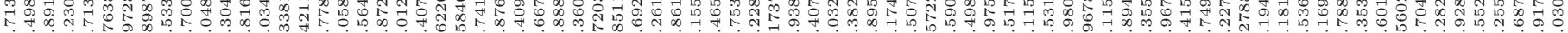

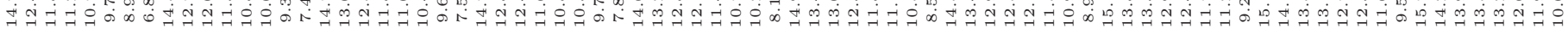

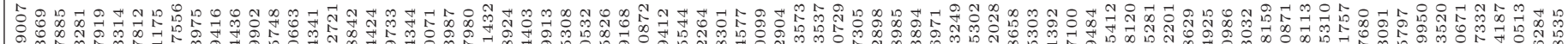

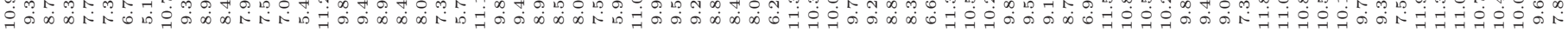

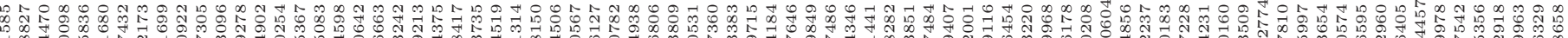

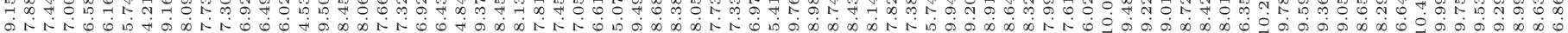

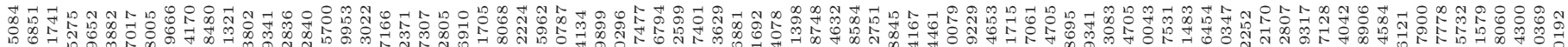

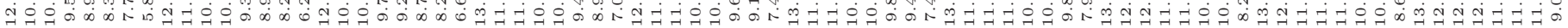

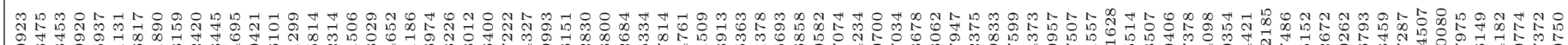

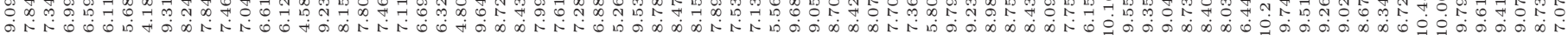

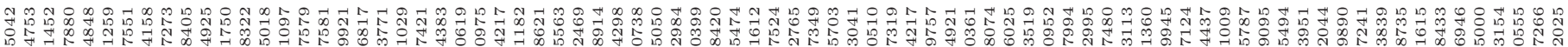

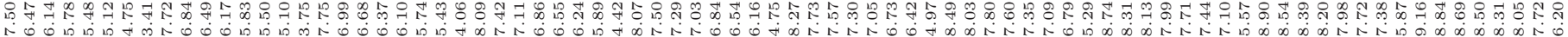
$\begin{array}{ll}0 & \\ 0 & 0\end{array}$

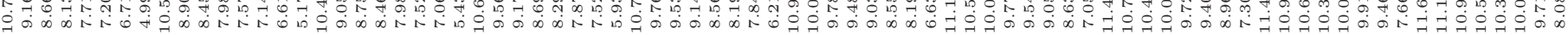

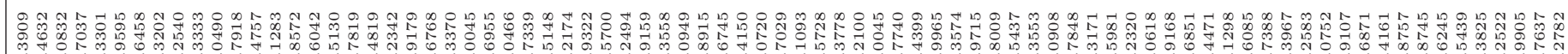
N

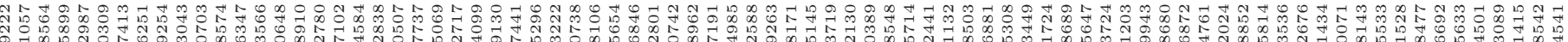
$\begin{array}{lll} & \\ 0\end{array}$

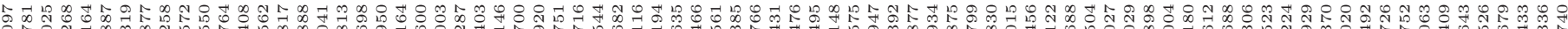

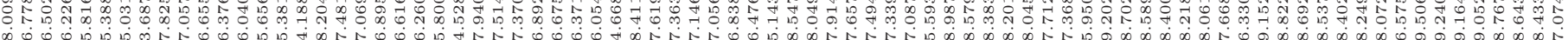

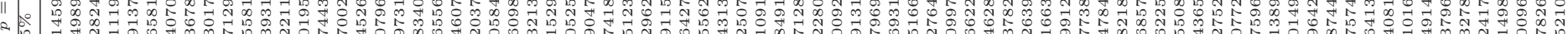

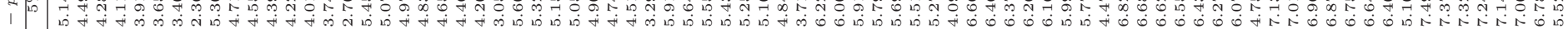

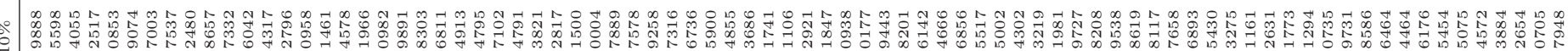

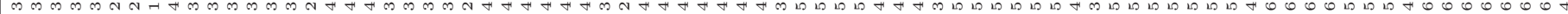

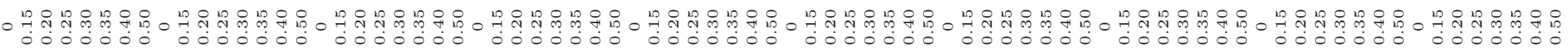
2 (1) N $\infty$ $+$ 10 


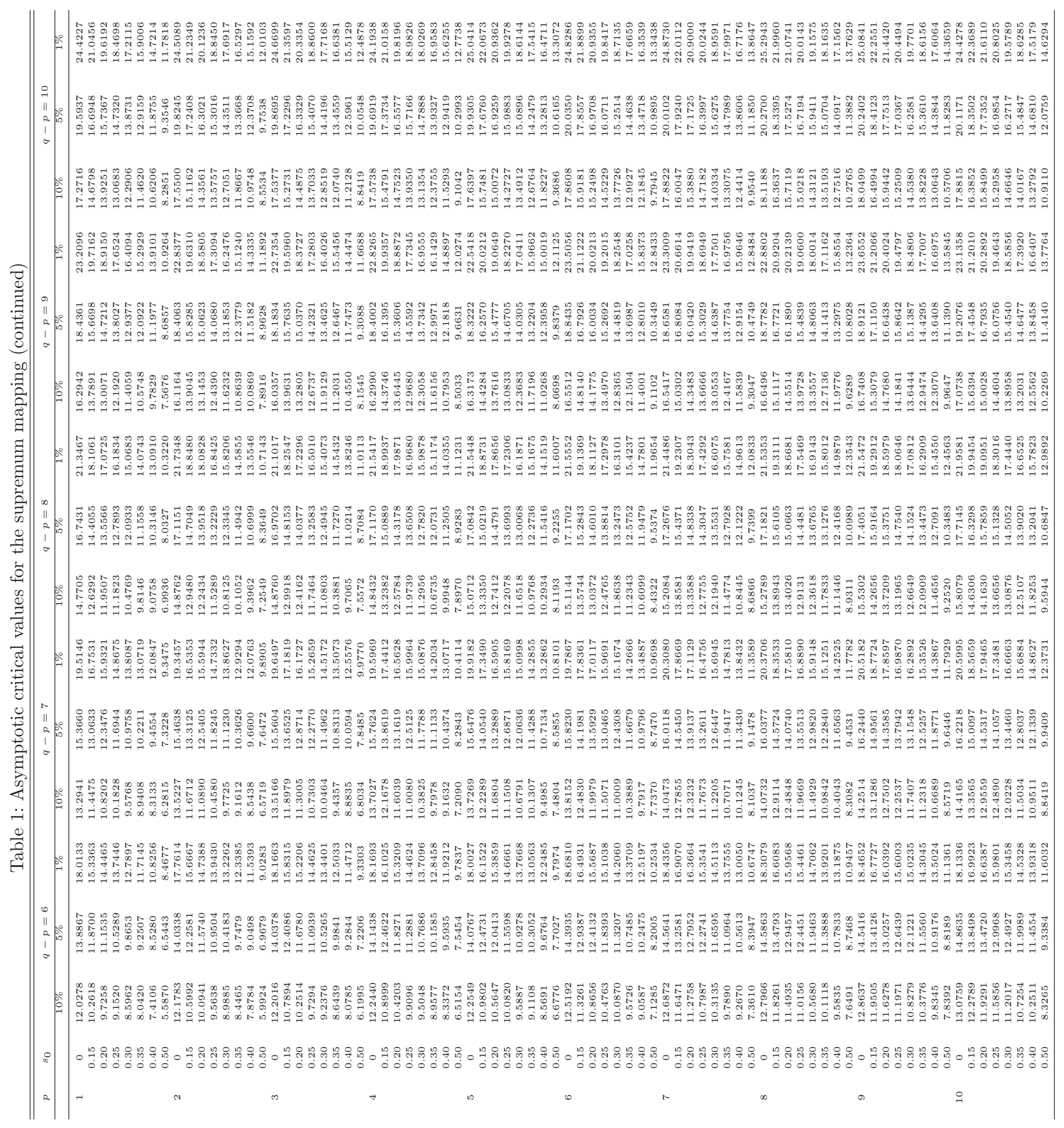




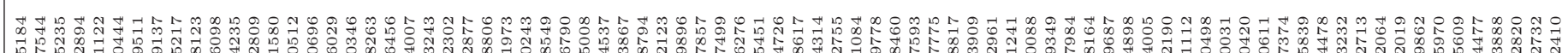

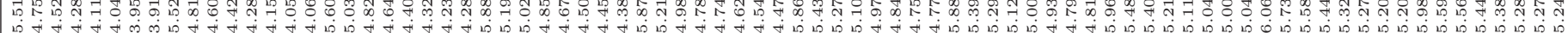

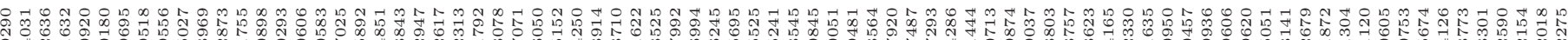

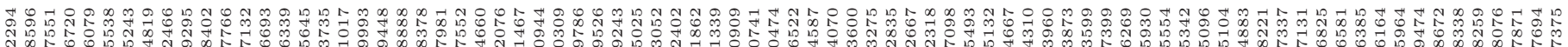

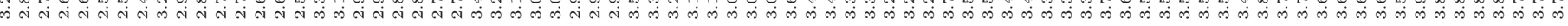

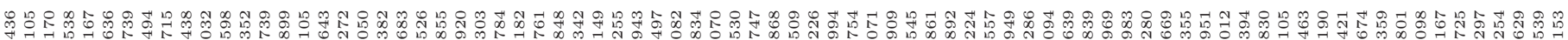

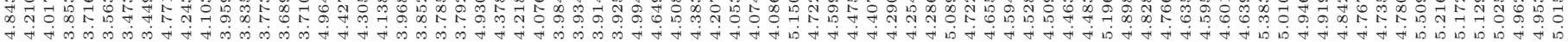

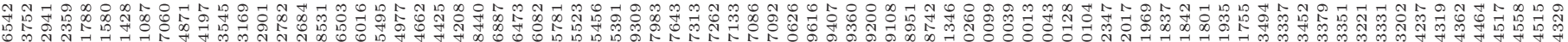

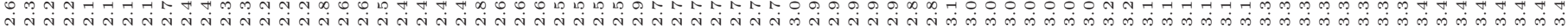

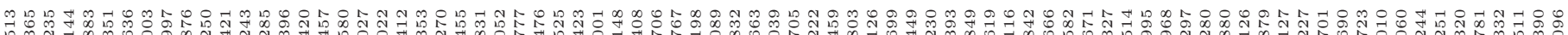
范梁

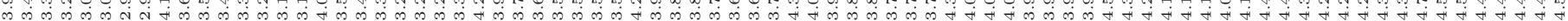

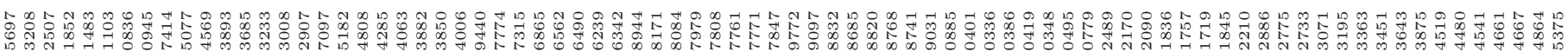

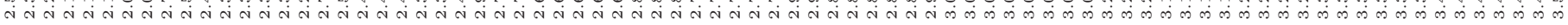

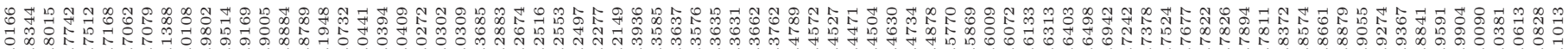

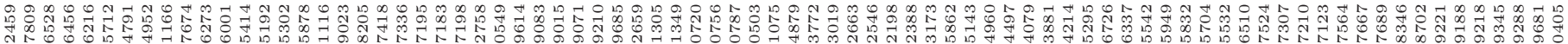

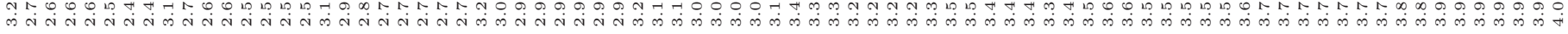

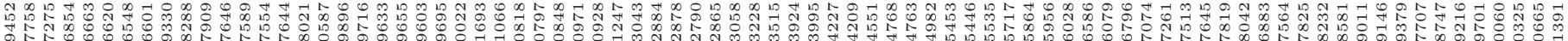

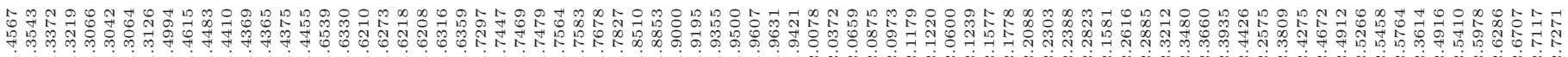

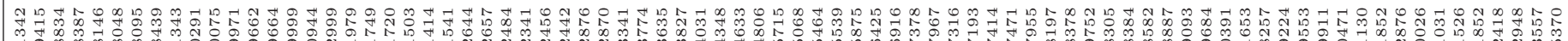

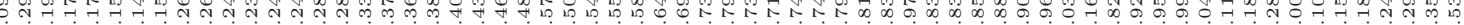

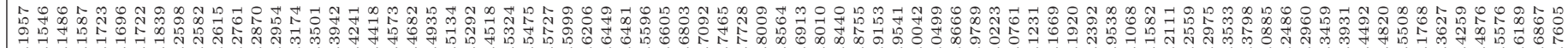

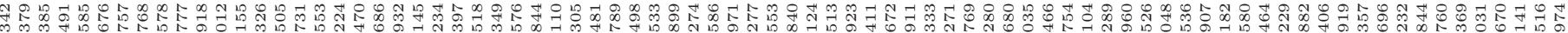
等

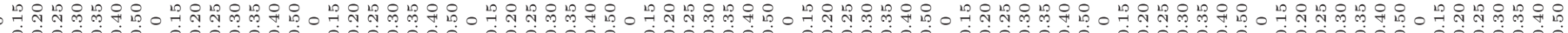

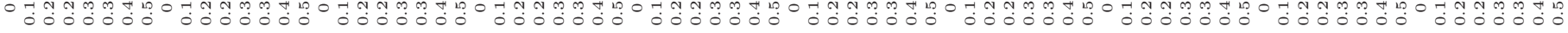
2

八

$\infty$

。

N

$\infty$

욱 


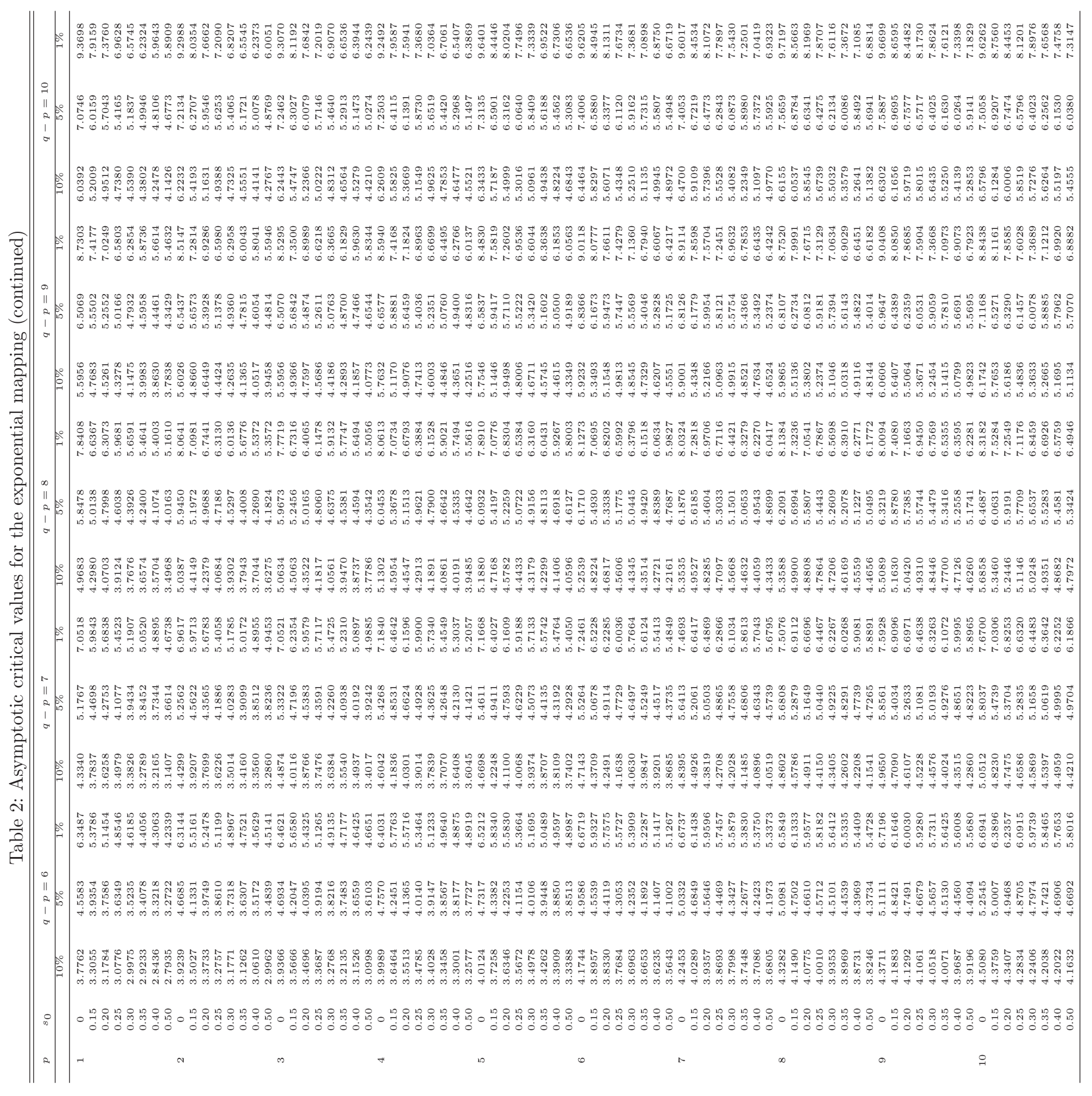




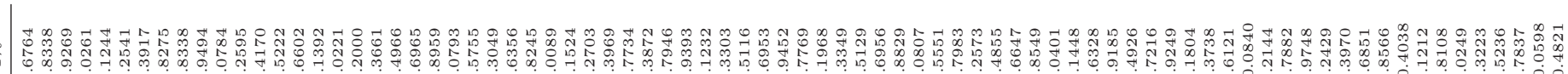

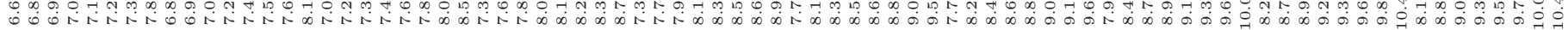

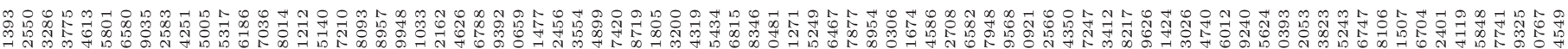

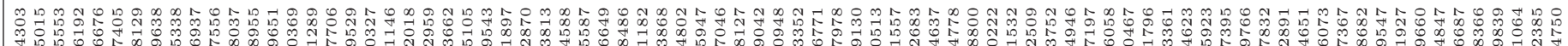

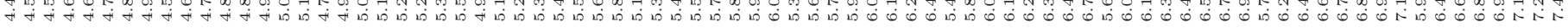

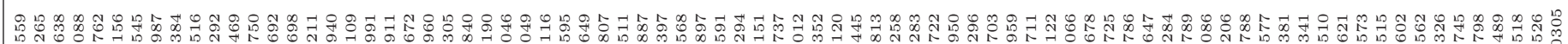
10

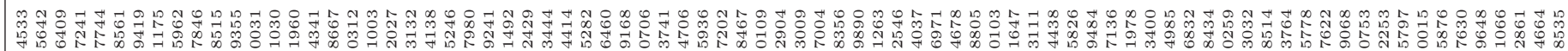

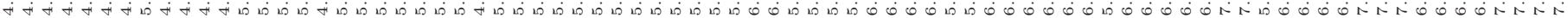

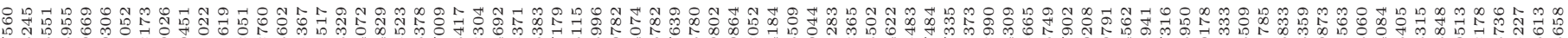

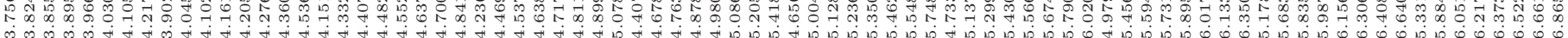

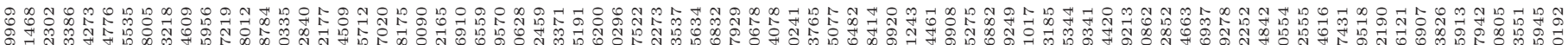

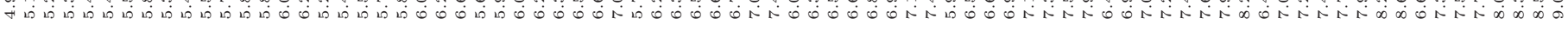

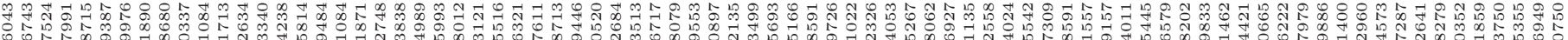

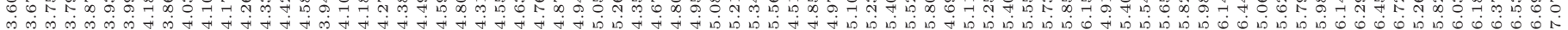

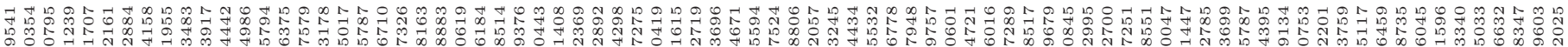

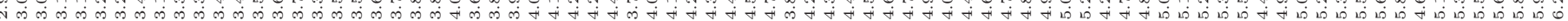

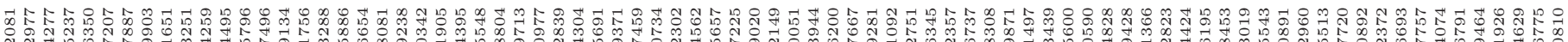

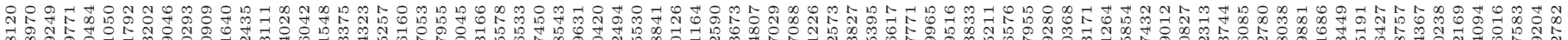

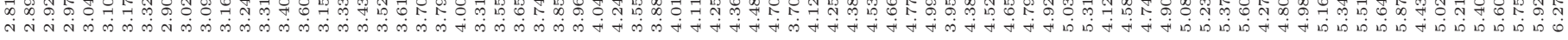

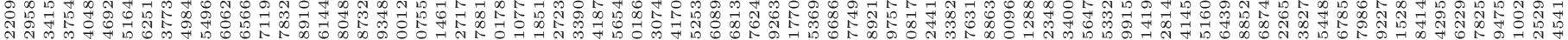

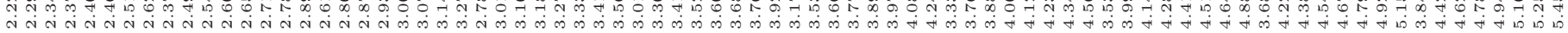

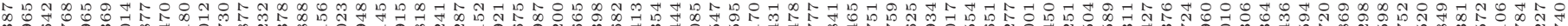

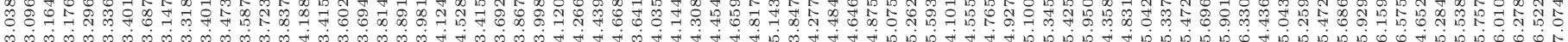

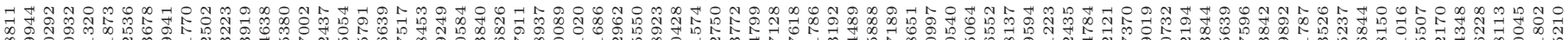

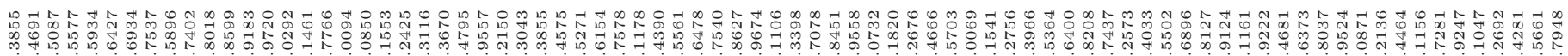

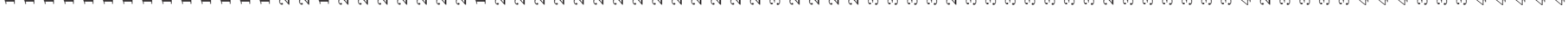

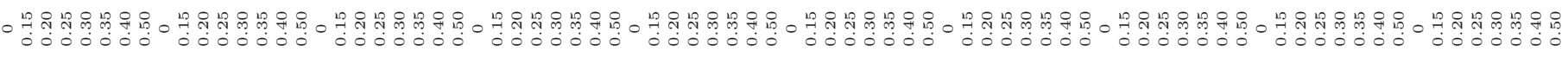
N $\infty$
$+$

D

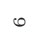

$\checkmark$

$\infty$

$\circ$

욱 


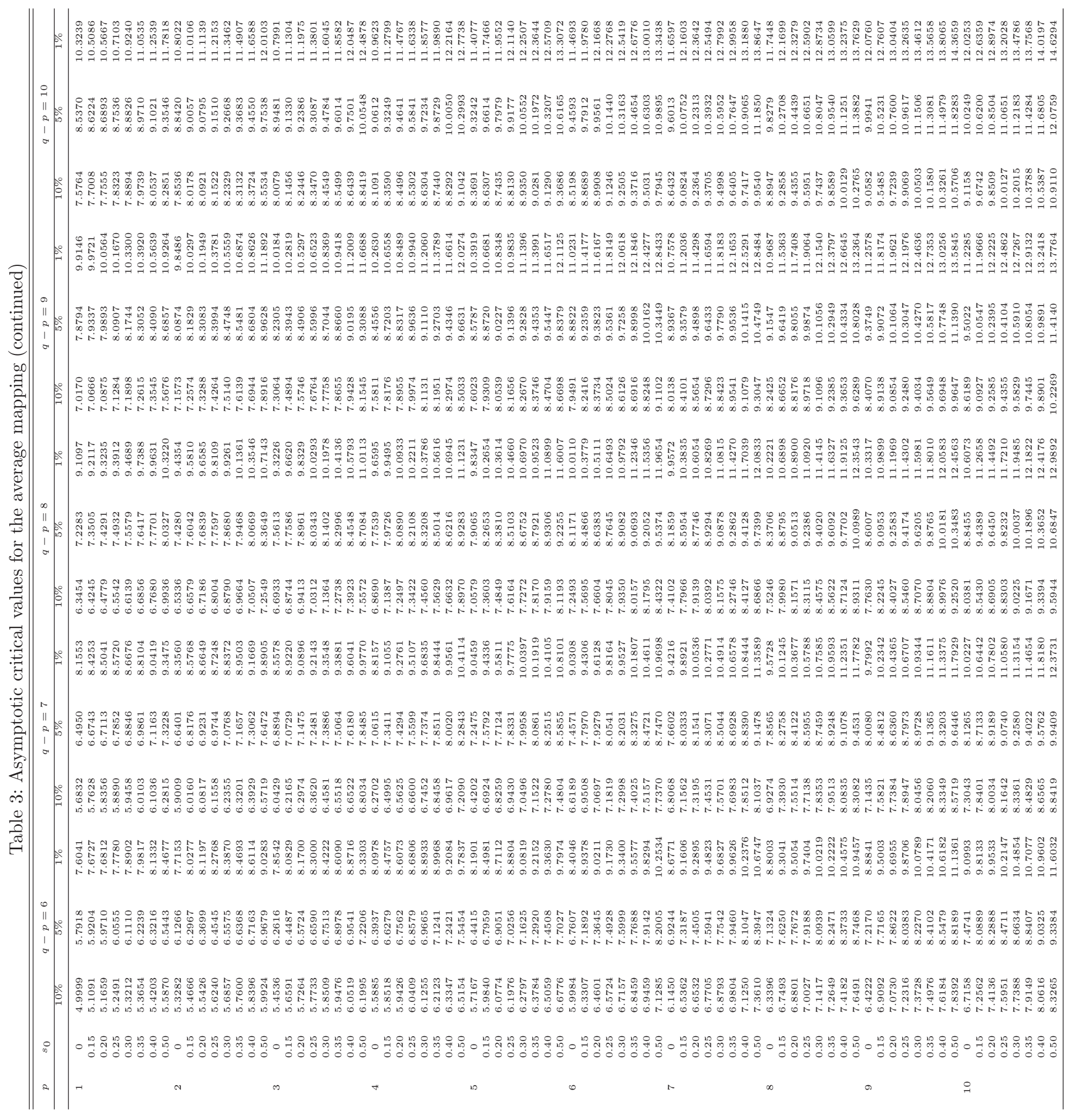


Table 4: Data Generating Processes

\begin{tabular}{cccc}
\hline \hline & $H_{0}^{S S}$ & $H_{A}^{I}$ & $H_{A}^{O}$ \\
\hline DGP1 & $\theta_{1}=\theta_{2}=0$ & & $\alpha=0$ \\
DGP2 & $\theta_{1}=\theta_{2}=0.4$ & & $\alpha=0$ \\
DGP3 & $\theta_{1}=\theta_{2}=0.8$ & & $\alpha=0$ \\
DGP4 & & $\theta_{1}=0, \theta_{2}=0.4$ & $\alpha=0$ \\
DGP5 & & $\theta_{1}=0, \theta_{2}=0.8$ & $\alpha=0$ \\
DGP6 & & $\theta_{1}=0.4, \theta_{2}=0.8$ & $\alpha=0$ \\
DGP7 & $\theta_{1}=\theta_{2}=0.4$ & & $\alpha=0.5$ \\
DGP8 & $\theta_{1}=\theta_{2}=0.4$ & & $\alpha=0.9$ \\
DGP9 & $\theta_{1}=\theta_{2}=0.4$ & & $\alpha=-0.5$ \\
DGP10 & $\theta_{1}=\theta_{2}=0.4$ & & $\alpha=-0.9$ \\
\hline
\end{tabular}

Table 5: Rejection Frequencies for General Tests of Structural Change

\begin{tabular}{|c|c|c|c|c|c|c|c|}
\hline DGP & Size $(\%)$ & supIPSC & aveIPSC & $\operatorname{expIPSC}$ & supIPSCM & aveIPSCM & $\operatorname{expIPSCM}$ \\
\hline \multirow[t]{3}{*}{ DGP1 } & 1 & 0.0155 & 0.0145 & 0.0160 & 0.0770 & 0.0435 & 0.0590 \\
\hline & 5 & 0.0655 & 0.0615 & 0.0655 & 0.1575 & 0.1035 & 0.1275 \\
\hline & 10 & 0.1255 & 0.1255 & 0.1270 & 0.2295 & 0.1720 & 0.1870 \\
\hline \multirow[t]{3}{*}{ DGP2 } & 1 & 0.0145 & 0.0155 & 0.0145 & 0.0750 & 0.0385 & 0.0595 \\
\hline & 5 & 0.0660 & 0.0545 & 0.0610 & 0.1520 & 0.1005 & 0.1225 \\
\hline & 10 & 0.1260 & 0.1215 & 0.1245 & 0.2330 & 0.1735 & 0.1925 \\
\hline \multirow[t]{3}{*}{ DGP3 } & 1 & 0.0225 & 0.0140 & 0.0165 & 0.0835 & 0.0465 & 0.0575 \\
\hline & 5 & 0.0685 & 0.0670 & 0.0690 & 0.1525 & 0.1050 & 0.1215 \\
\hline & 10 & 0.1195 & 0.1225 & 0.1210 & 0.2300 & 0.1680 & 0.1875 \\
\hline \multirow{3}{*}{ DGP4 } & 1 & 0.1465 & 0.1590 & 0.1515 & 0.4070 & 0.3190 & 0.3560 \\
\hline & 5 & 0.3955 & 0.3980 & 0.4010 & 0.5985 & 0.5365 & 0.5635 \\
\hline & 10 & 0.5590 & 0.5980 & 0.5885 & 0.7050 & 0.6840 & 0.6925 \\
\hline \multirow[t]{3}{*}{ DGP5 } & 1 & 0.9780 & 0.9710 & 0.9765 & 0.9990 & 0.9955 & 0.9980 \\
\hline & 5 & 0.9985 & 0.9980 & 0.9985 & 1.0000 & 0.9995 & 0.9995 \\
\hline & 10 & 1.0000 & 0.9995 & 1.0000 & 1.0000 & 0.9995 & 1.0000 \\
\hline \multirow[t]{3}{*}{ DGP6 } & 1 & 0.4095 & 0.3825 & 0.3995 & 0.7020 & 0.6130 & 0.6675 \\
\hline & 5 & 0.7040 & 0.6685 & 0.6885 & 0.8450 & 0.8020 & 0.8225 \\
\hline & 10 & 0.8170 & 0.8120 & 0.8220 & 0.8970 & 0.8810 & 0.8870 \\
\hline \multirow{3}{*}{ DGP7 } & 1 & 0.2945 & 0.1835 & 0.2365 & 0.5535 & 0.3680 & 0.4780 \\
\hline & 5 & 0.5320 & 0.4005 & 0.4675 & 0.7090 & 0.5360 & 0.6270 \\
\hline & 10 & 0.6690 & 0.5660 & 0.6050 & 0.7805 & 0.6515 & 0.7145 \\
\hline \multirow{3}{*}{ DGP8 } & 1 & 0.6080 & 0.4565 & 0.5340 & 0.8205 & 0.6555 & 0.7645 \\
\hline & 5 & 0.8120 & 0.7000 & 0.7620 & 0.9025 & 0.7955 & 0.8580 \\
\hline & 10 & 0.8910 & 0.8330 & 0.8645 & 0.9370 & 0.8720 & 0.9040 \\
\hline \multirow{3}{*}{ DGP9 } & 1 & 0.3970 & 0.2385 & 0.3265 & 0.5580 & 0.3235 & 0.4630 \\
\hline & 5 & 0.6200 & 0.4825 & 0.5590 & 0.7005 & 0.4990 & 0.6150 \\
\hline & 10 & 0.7390 & 0.6415 & 0.6890 & 0.7810 & 0.6575 & 0.7180 \\
\hline \multirow{3}{*}{ DGP10 } & 1 & 0.7975 & 0.6695 & 0.7535 & 0.8515 & 0.6685 & 0.7930 \\
\hline & 5 & 0.9420 & 0.8790 & 0.9175 & 0.9290 & 0.8545 & 0.9055 \\
\hline & 10 & 0.9730 & 0.9525 & 0.9650 & 0.9625 & 0.9435 & 0.9580 \\
\hline
\end{tabular}


Table 6: Rejection Frequencies for Tests of Structural Change in the Parameters

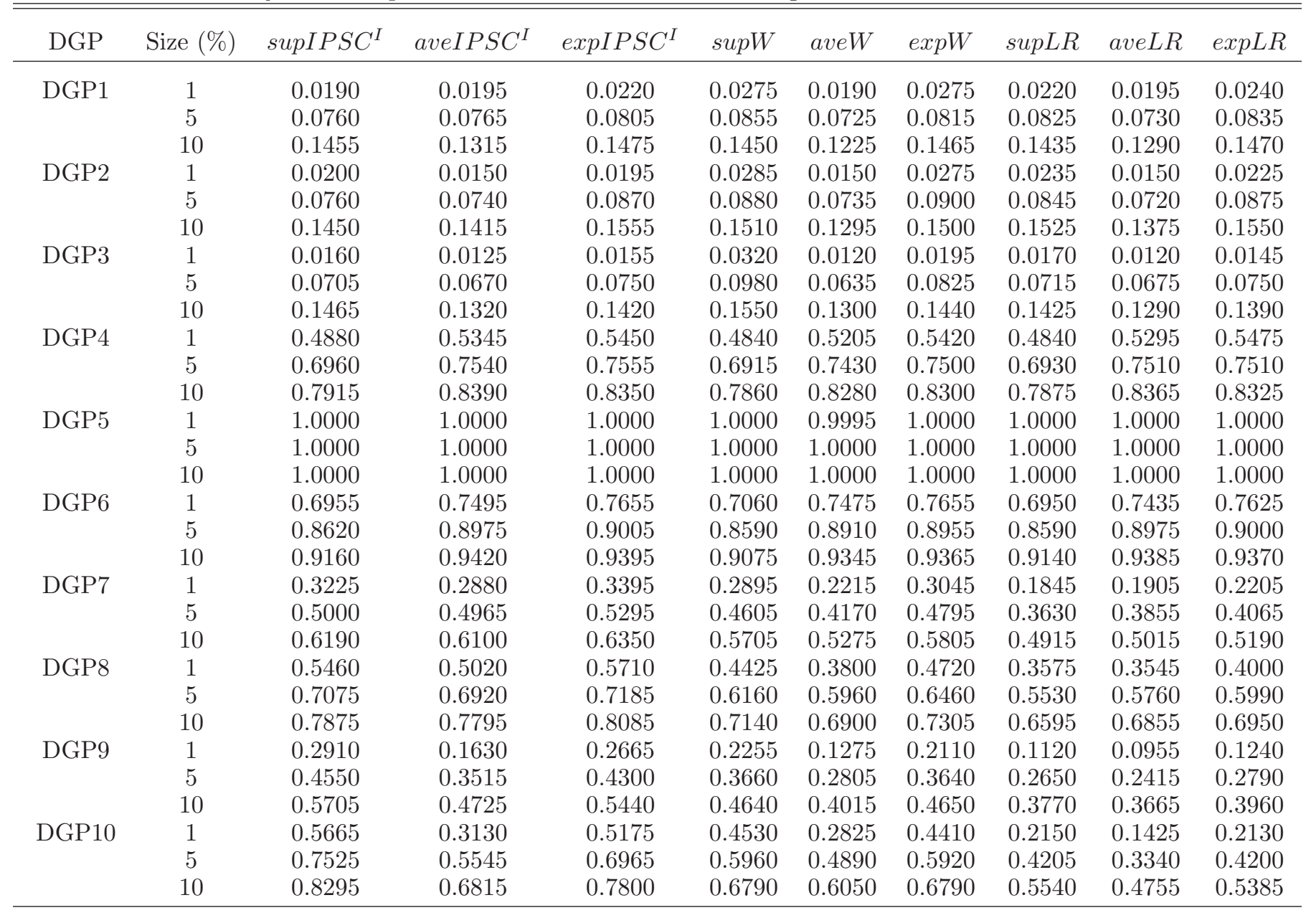


Table 7: Rejection Frequencies for Tests of Structural Change in the Overidentifying Restrictions

\begin{tabular}{cccccccc}
\hline \hline DGP & Size $(\%)$ & supIPSC & aveIPSC & expIPSC & supO & aveO & expO \\
\hline DGP1 & 1 & 0.0195 & 0.0195 & 0.0175 & 0.0020 & 0.0075 & 0.0050 \\
& 5 & 0.0715 & 0.0630 & 0.0675 & 0.0210 & 0.0420 & 0.0390 \\
DGP2 & 10 & 0.1290 & 0.1240 & 0.1320 & 0.0595 & 0.1005 & 0.0865 \\
& 1 & 0.0280 & 0.0145 & 0.0220 & 0.0040 & 0.0085 & 0.0085 \\
DGP3 & 10 & 0.0825 & 0.0630 & 0.0810 & 0.0270 & 0.0450 & 0.0440 \\
& 1 & 0.1300 & 0.1225 & 0.1300 & 0.0595 & 0.0955 & 0.0865 \\
& 5 & 0.0270 & 0.0170 & 0.0240 & 0.0055 & 0.0090 & 0.0075 \\
DGP4 & 10 & 0.0790 & 0.0650 & 0.0780 & 0.0295 & 0.0490 & 0.0440 \\
& 1 & 0.0300 & 0.1230 & 0.1420 & 0.0630 & 0.1035 & 0.0890 \\
& 5 & 0.0970 & 0.0170 & 0.0250 & 0.0050 & 0.0070 & 0.0080 \\
DGP5 & 10 & 0.1650 & 0.1370 & 0.0925 & 0.0360 & 0.0460 & 0.0445 \\
& 1 & 0.2930 & 0.1420 & 0.1640 & 0.0810 & 0.0985 & 0.1075 \\
DGP6 & 10 & 0.5165 & 0.3420 & 0.4975 & 0.1605 & 0.0805 & 0.1725 \\
& 1 & 0.6380 & 0.5000 & 0.6200 & 0.5305 & 0.2630 & 0.4040 \\
& 5 & 0.0415 & 0.0220 & 0.0390 & 0.0085 & 0.01300 & 0.5420 \\
DGP7 & 10 & 0.1100 & 0.0740 & 0.0960 & 0.0505 & 0.0515 & 0.06145 \\
& 1 & 0.6080 & 0.1430 & 0.1695 & 0.0925 & 0.1055 & 0.1140 \\
& 5 & 0.7755 & 0.5790 & 0.6340 & 0.0425 & 0.1725 & 0.1290 \\
DGP8 & 10 & 0.8370 & 0.8580 & 0.7965 & 0.2380 & 0.5415 & 0.4740 \\
& 1 & 0.7955 & 0.8080 & 0.8695 & 0.4380 & 0.7255 & 0.6790 \\
DGP9 & 10 & 0.8935 & 0.9135 & 0.8230 & 0.0850 & 0.3275 & 0.2375 \\
& 1 & 0.9275 & 0.9525 & 0.9510 & 0.3620 & 0.7540 & 0.6755 \\
DGP10 & 5 & 0.8780 & 0.8900 & 0.9065 & 0.0960 & 0.4185 & 0.2975 \\
& 10 & 0.9565 & 0.9625 & 0.9660 & 0.4575 & 0.8230 & 0.7810 \\
& 1 & 0.9760 & 0.9845 & 0.9840 & 0.7150 & 0.9445 & 0.9245 \\
& 10 & 0.9950 & 0.9955 & 0.9960 & 0.1140 & 0.6610 & 0.4790 \\
& 0.9980 & 1.0000 & 0.9995 & 0.6080 & 0.9780 & 0.9470 \\
& 0.9995 & 1.0000 & 1.0000 & 0.8645 & 0.9970 & 0.9925 \\
\hline
\end{tabular}

